\title{
RETHINIKING THE CARBON ECONOMY: \\ COMBINED ELECTROCHEMICAL AND BIOLOGICAL REDUCTION FOR CARBON DIOXIDE CAPTURE AND VALORIZATION
}

\author{
by \\ JOSHUA JACK \\ B.Sc., University of Massachusetts, Amherst, 2014 \\ M.Sc., University of Massachusetts, Amherst, 2015
}

\author{
A thesis submitted to the \\ Faculty of the Graduate School of the \\ University of Colorado in partial fulfillment \\ of the requirement for the degree of \\ Doctor of Philosophy
}

Department of Civil, Environmental, and Architectural Engineering

2019 
This thesis entitled:

Rethinking the carbon economy: Combined electrochemical and biological reduction for carbon dioxide capture and valorization

written by Joshua Jack

has been approved for the Department of Civil, Environmental, and Architectural Engineering

Sherri Cook, Ph.D.

JoAnn Silverstein, Ph.D.

Angela Bielefeldt, Ph.D.

Pin-Ching Maness, Department of Energy

Zhiyong (Jason) Ren, Ph.D

Date

The final copy of this thesis has been examined by the signatories, and we find that both the content and the form meet acceptable presentation standards of scholarly work in the above mentioned discipline. 
Jack, Joshua (Ph.D., Environmental Engineering)

Rethinking the carbon economy: Combined electrochemical and biological reduction for carbon dioxide capture and valorization

Thesis directed by Professor Jason Ren

\begin{abstract}
Carbon is a primary component of most present-day commodities and can be viewed as the backbone of our global economy. Carbon dioxide is considered one of the most abundant forms of carbon on earth and its continued buildup in the atmosphere has reached concentrations of around $410 \mathrm{ppm}$ (ca. 32000 gigatons) [1]. Though many fear the impending effects of this accumulation on global climate patterns, others have viewed this plentiful compound as a cheap and largely untapped resource. Engineered carbon dioxide reduction to high energy density fuels and chemicals presents an economically sound approach to mitigate climate change and yield tremendous environmental and societal benefits. Due to the rapid decrease in the cost of renewable energy, it is practical to design devices that may use solar or wind power to simultaneously drive $\mathrm{CO}_{2}$ sequestration and transformation. The injection of an electron into $\mathrm{CO}_{2}$, however, requires highly negative potentials (large energy investment) and the kinetics are quite sluggish under standard conditions. Coupling $\mathrm{CO}_{2}$-fixing autotrophic microorganisms with electrochemical systems is a sustainable approach for engineered (non-photosynthetic) $\mathrm{CO}_{2}$ reduction, however, this is a nascent field and only a few approaches have been presented to date. In this work, I conducted multi-disciplinary research in the fields of microbiology, material science and electrochemistry to develop a two-stage biohybrid system that feeds electro-
\end{abstract}


generated syngas produced from a $\mathrm{CO}_{2}$ electrolysis cell to a downstream bioreactor for highly efficient conversion of $\mathrm{CO}_{2}$ to value-added carbon products. The specific studies presented in this dissertation consist of an investigation of tuning key syngas fermentation parameters of Clostridium ljungdahlii to create desired metabolites, development of a versatile carbon material from waste-grown fungal biomass, synthesis and characterization of novel $\mathrm{CO}_{2}$ reduction catalysts, and design and optimization of a two-stage biohybrid system for engineered carbon dioxide reduction. My studies conclude with future research focused on developing electro catalysts for electrochemical hydrogen production to be used a feedstock for a downstream catalytic hydrogenation process as well as other work involving the creation of functionalized hollow-fiber membranes for dual $\mathrm{CO}_{2}$ conversion and delivery. 


\section{Acknowledgements}

I would sincerely like to express that I only take a small fraction of credit for the great accomplishment of completing this dissertation and other doctoral requirements, as I could not have done it without all of the amazing and wonderful people who have relentlessly showed their support along the way. Firstly, I would like to thank my advisor Dr. Jason Ren for his guidance, encouragement and confidence in allowing me to pursue my research ideas and for always keeping my best interests in mind. I feel very lucky to have had you as my advisor and as a friend. Moreover, I would like to thank everyone in the Ren research group for their technical and life advice along the way. I would also like to thank Pin-Ching Maness who made NREL feel like a home to me and graciously welcomed me into her lab as well as supported me throughout my graduate studies. In addition, I am extremely grateful for all the help and encouragement I received while working at NREL and am glad I got the opportunity to know many of the scientists and establish life-long friendships. I would also like to thank and acknowledge Eunsol Park for her help with material characterization described in Chapters 4 and 6. To all my committee members, Dr. JoAnn Silverstein, Dr. Angela Bielefeldt, and Dr. Sherri Cook, thank you for all your time, welcomed feedback, and advice. Lastly, I would like to thank all of my family and close friends who have always showed me love and support. I am truly blessed to have you all in my life. 


\section{Table of Contents}

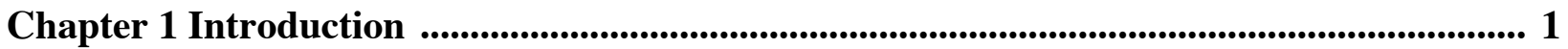

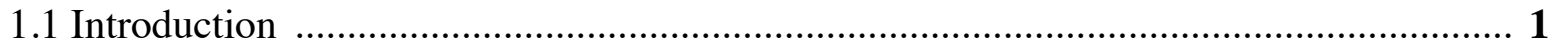

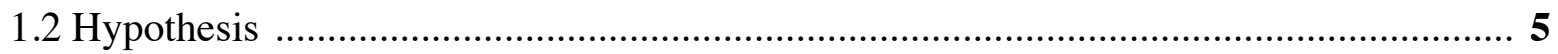

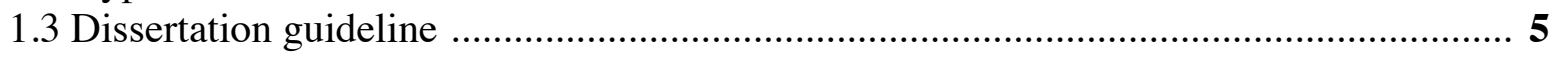

Chapter 2 Directing $C$.ljungdahlii fermentation via $\mathrm{H}_{2} / \mathrm{CO}$ syngas ratio $\quad$............................... 7

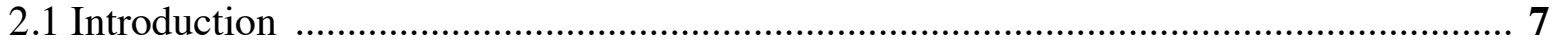

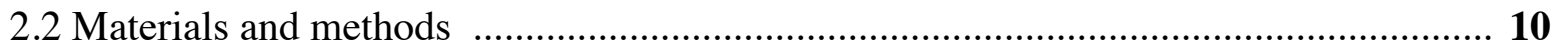

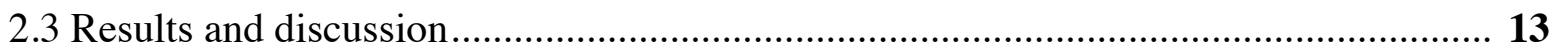

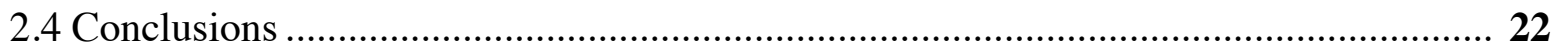

Chapter 3 Production of magnetic biochar from waste-derived fungal biomass ................. 24

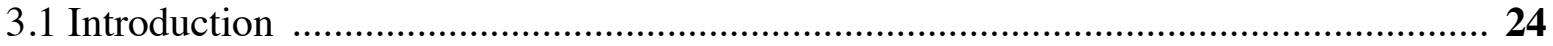

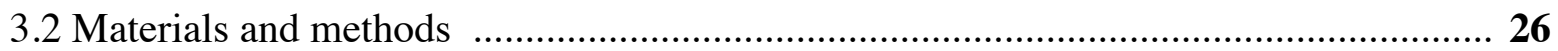

3.3 Results and discussion .................................................................................... 31

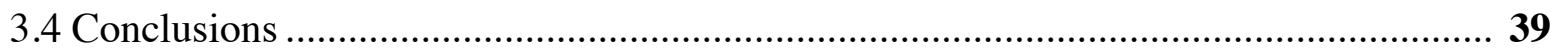

Chapter 4 Optimization of electrocatalytic syngas generation on CoTPP ......................... 41

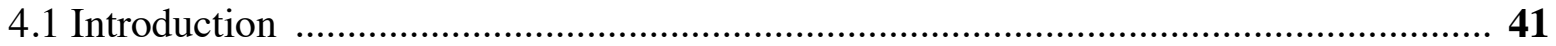

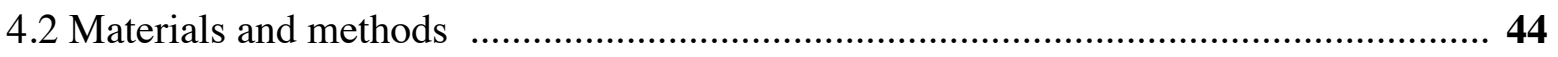

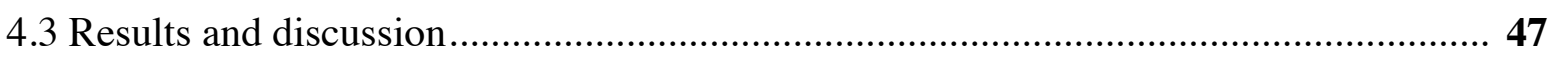

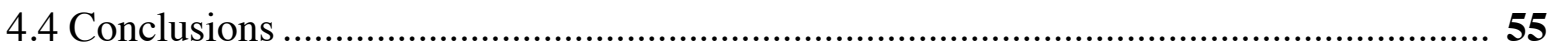

Chapter 5 Two-stage biohybrid system for $\mathrm{CO}_{2}$ valorization ........................................... 56

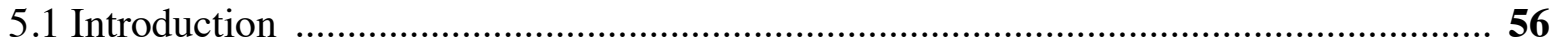

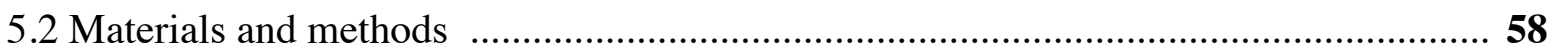

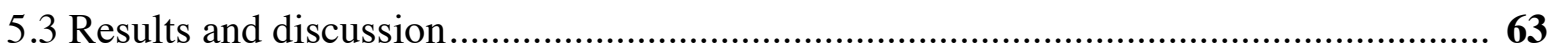

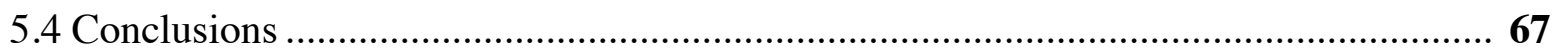

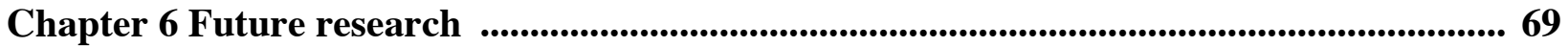

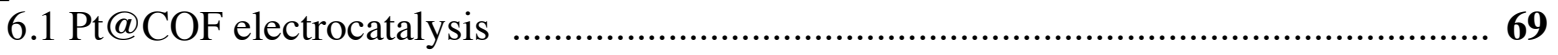

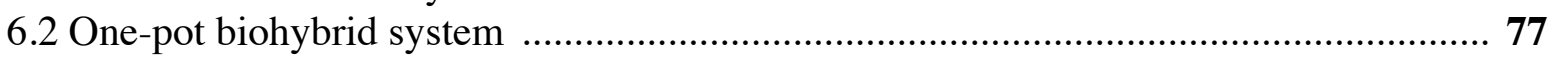

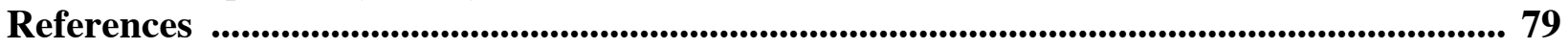

Appendix A 


\section{List of Tables}

Table 1 Summary of biochar physiochemical properties ....................................................... 33 


\section{List of Figures}

Figure 1: Two-stage biohybrid system schematic for $\mathrm{CO}_{2}$ valorization ................................... 4

Figure 2: Clostridium ljungdahlii maximum reported ethanol to acetate concentration ratio vs.

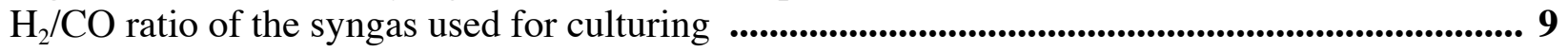

Figure 3: Clostridium ljungdahlii gas consumption rates ............................................................. 14

Figure 4: Comparison of Clostridium ljungdahlii $\mathrm{CO}_{2}$ production .................................................. 15

Figure 5: Impact of $\mathrm{H}_{2} / \mathrm{CO}$ ratio on product formation .......................................................... 17

Figure 6: Comparison of Clostridium ljungdahlii acetate and ethanol production ..................... 18

Figure 7: Clostridium ljungdahlii growth on syngas ............................................................... 21

Figure 8: Clostridium ljungdahlii product yield and efficiencies ................................................. 22

Figure 9: Flow diagram for magnetic biochar production ....................................................... 28

Figure 10: Biochar production and characterization images ....................................................... 31

Figure 11: Neurospora Crassa growth and nutrient uptake ................................................. 32

Figure 12: Fungal biochar material characterization summary ................................................. 35

Figure 13: Adsorption isotherms and error analysis of phosphorus on fungi derived biochars 37

Figure 14: Phosphorus loaded biochar FTIR analysis summary .................................................. 38

Figure 15: Summary of synthesis reactions for $\mathrm{Co}(\mathrm{o}-\mathrm{OH}) \mathrm{TPP}$ and $\mathrm{Co}\left(\mathrm{o}-\mathrm{OCH}_{3}\right) \mathrm{TPP}$.............. 45

Figure 16: $\mathrm{CO}_{2}$ reduction capabilities of modified cobalt porphyrin structures .......................... 49

Figure 17: Cobalt porphyrin catalyst preparative scale electrolysis $\quad$....................................... 50

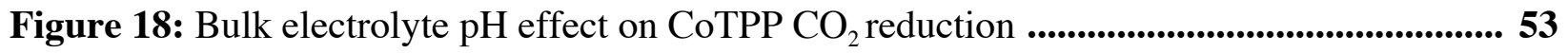

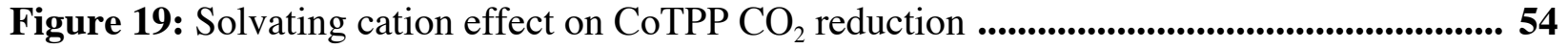

Figure 20: Sodium stabilization effect on $\mathrm{COTPP}_{2}$ reduction .................................................. 55

Figure 21: Detailed schematic of $\mathrm{CO}_{2}$ electrolyzer and associated reactions ............................ 59

Figure 22: Schematic of two-stage biohybrid operating process ................................................... 61

Figure 23: Gas production and faradaic efficiency at variable current densities ........................ 63

Figure 24: $\mathrm{CO}_{2}$ electrolysis current density vs. time ................................................................ 64

Figure 25: Biohybrid system microbial growth and system stability $\quad$............................................. 65

Figure 26: C.ljungdahlii acetate and ethanol production in two-stage biohybrid system ............66

Figure 27: Comparison of C.ljungdahlii PtA mutant and wild-type product generation............. 67 
Figure 28: Pt@COF compound and material characterization summary .................................. 73

Figure 29: Pt@COF electrochemical hydrogen evolution summary ......................................... 76

Figure 30: One-pot biohybrid system schematic ................................................................................. 78 


\section{CHAPTER 1}

\section{INTRODUCTION}

\subsection{Rational}

Carbon dioxide $\left(\mathrm{CO}_{2}\right)$ is considered one of the most abundant sources of carbon on earth. The current $\mathrm{CO}_{2}$ concentration in the atmosphere is approximately $410 \mathrm{ppm}$, which corresponds to ca. 3,200 gigatons of $\mathrm{CO}_{2}$ [1]. In addition, more than 40 billion tons of $\mathrm{CO}_{2}$ are released from human activities every year, leading to a concentration increase of ca. 2 ppm per year [2]. Although this rapid accumulation of $\mathrm{CO}_{2}$ has prompted various global environmental and societal concerns, it may also be considered a renewable source of a low-cost building block. Carbon is the primary component of fuels, chemicals and materials that are used everyday and thus compose a large section of the global economy. By 2050, the human population is expected to increase from ca. 7.6 to 9.8 billion people, creating a huge increase in demand for such commodities [3].

The utilization of $\mathrm{CO}_{2}$ as a renewable carbon feedstock for fuels and chemicals could simultaneously help satisfy this demand while actively mitigating global $\mathrm{CO}_{2}$ emissions. Technologies that can accomplish this task could pave the way for a closed-carbon economy while yielding tremendous social and environmental benefits. Furthermore, such devices can achieve excellent economic profits by leveraging the low-cost of $\mathrm{CO}_{2}$ as a feedstock for high value products and by taking advantage of governmental carbon capture incentives [4].

In the past, the majority of techniques that could convert $\mathrm{CO}_{2}$ into valued carbon products have involved photosynthetic carbon assimilation via plants, algae or cyanobacteria followed by biomass deconstruction and upgrade processes [5]. The commercialization of such systems is, however, fundamentally limited by low-energy to biomass conversions as well as large water, land, and nutrient requirements that will undoubtedly become more precarious with growing populations [6-9]. 
With that in mind, incredible efforts have been made to develop other engineered carbon reduction platforms to accomplish conversion of $\mathrm{CO}_{2}$ into products. These include nonbiological processes such as thermocatalysis, photocatalysis and electrocatalysis, as well as nonphotosynthetic biocatalytic processes. To date, the bulk of these devices have been challenged by the large energy requirement to reduce $\mathrm{CO}_{2}$ to organic molecules $[10,11]$. In recent years, cheap and abundant renewable energy sources such as solar or wind have become an attractive supply of reducing power for $\mathrm{CO}_{2}$ reduction. As photocatalytic and thermocatalytic techniques directly convert sunlight to either free electrons or heat, a greater abundance of electrons does not directly benefit them [11]. In contrast, the increased accessibility of cheap (renewable) electrons has greatly improved the viability for scale-up of approaches that involve electrocatalytic or nonphotosynthetic biocatalytic $\mathrm{CO}_{2}$ reduction.

Abiotic eletrocatalytic $\mathrm{CO}_{2}$ reduction utilizes the electrons from an external source (i.e. solar or wind) as the reducing power to transform $\mathrm{CO}_{2}$ to a variety of products on the electrode surface. Since $\mathrm{CO}_{2}$ is extremely stable at standard conditions, an electrocatalyst is typically employed at the electrode to mediate the reaction [12]. Present approaches for electrochemical $\mathrm{CO}_{2}$ reduction have been able to demonstrate excellent conversion rates and efficiencies for products like carbon monoxide (CO). For example, Peng et al. (2018) recently showed that their preferentially oriented silver nanoparticles could reduce $\mathrm{CO}_{2}$ to $\mathrm{CO}$ with a faradic efficiency of $96.7 \%$ at a low operating potential of $-0.69 \mathrm{~V}$ vs. RHE [13]. Although promising, products such as $\mathrm{CO}$ are of limited value as they require further processing to be used as fuels or upgraded into other value-added commodities. As a result, contemporary studies have focused on the formation of reduction products requiring four or more electrons (i.e. ethylene, oxalate) $[14,15]$. This work inherently poses additional challenges associated with higher over potentials and complex 
parallel reactions schemes that lead to decreased product specificity [16]. Presently, multielectron reduction of $\mathrm{CO}_{2}$ to products such as acetate, methane $\left(\mathrm{CH}_{4}\right)$ or ethanol $\left(\mathrm{C}_{2} \mathrm{H}_{5} \mathrm{OH}\right)$ has shown to be very energy inefficient and can only generate these products at low rates and yields $[17,18]$.

In contrast, non-photosynthetic biocatalytic approaches for $\mathrm{CO}_{2}$ reduction have demonstrated great overall energy efficiencies and can generate multi-reduction compounds with excellent product selectivity [19]. In the past decade, an approach known as microbial electrosynthesis (MES) has received a lot of attention [20-22]. In this process, chemolithoauthotrophs directly take electrons from an electrode using extra-cellular transfer (EET) mechanisms and use it as the reducing equivalent to convert $\mathrm{CO}_{2}$ into organic products. MES reactors can produce an extensive portfolio of compounds ranging from volatile fatty acids (formic acid, acetic acid, propionic acid, butyric acid) to alcohols (i.e. ethanol) $[19,22]$. Though encouraging, the production rates of MES technologies are orders of magnitudes lower than abiotic electrocatalytic systems. This is largely because catalysis in MES technologies is limited by usable electrode surface area, mass transfer of nutrients, and rates of microbial metabolic processes (i.e. EET) [19-23].

Combining the current understanding of electrocatalytic and non-photosynthetic biocatalytic $\mathrm{CO}_{2}$ conversion techniques, it seems that their limitations are quite complementary. As the EET rate in MES seems to be a limiting factor, circumventing these processes via an intermediate electron donor may be an effective solution. Accordingly, the most efficient approach for $\mathrm{CO}_{2}$ reduction to products may be a hybrid technique that combines high rates of electrochemical $\mathrm{CO}_{2}$ reduction to electro-generated intermediates followed by highly efficient biological upgrade of the intermediates to value-added products. The goal of this doctoral 
dissertation was to investigate abiotic and biological techniques for $\mathrm{CO}_{2}$ reduction to develop such a novel biohybrid platform. In the proposed two-stage design, microorganisms are separated from the electricity source, which enables optimized processes for both steps. In the first stage, $\mathrm{CO}_{2}$ is electrochemically converted to syngas (mixture of $\mathrm{CO} / \mathrm{H}_{2} / \mathrm{CO}_{2}$ ) in aninnovative gas diffusion $\mathrm{CO}_{2}$ electrolysis setup. The electro-generated syngas mixture is then delivered to the second stage where autotrophic bacteria utilize these components to synthesize biocommodities at high yields.

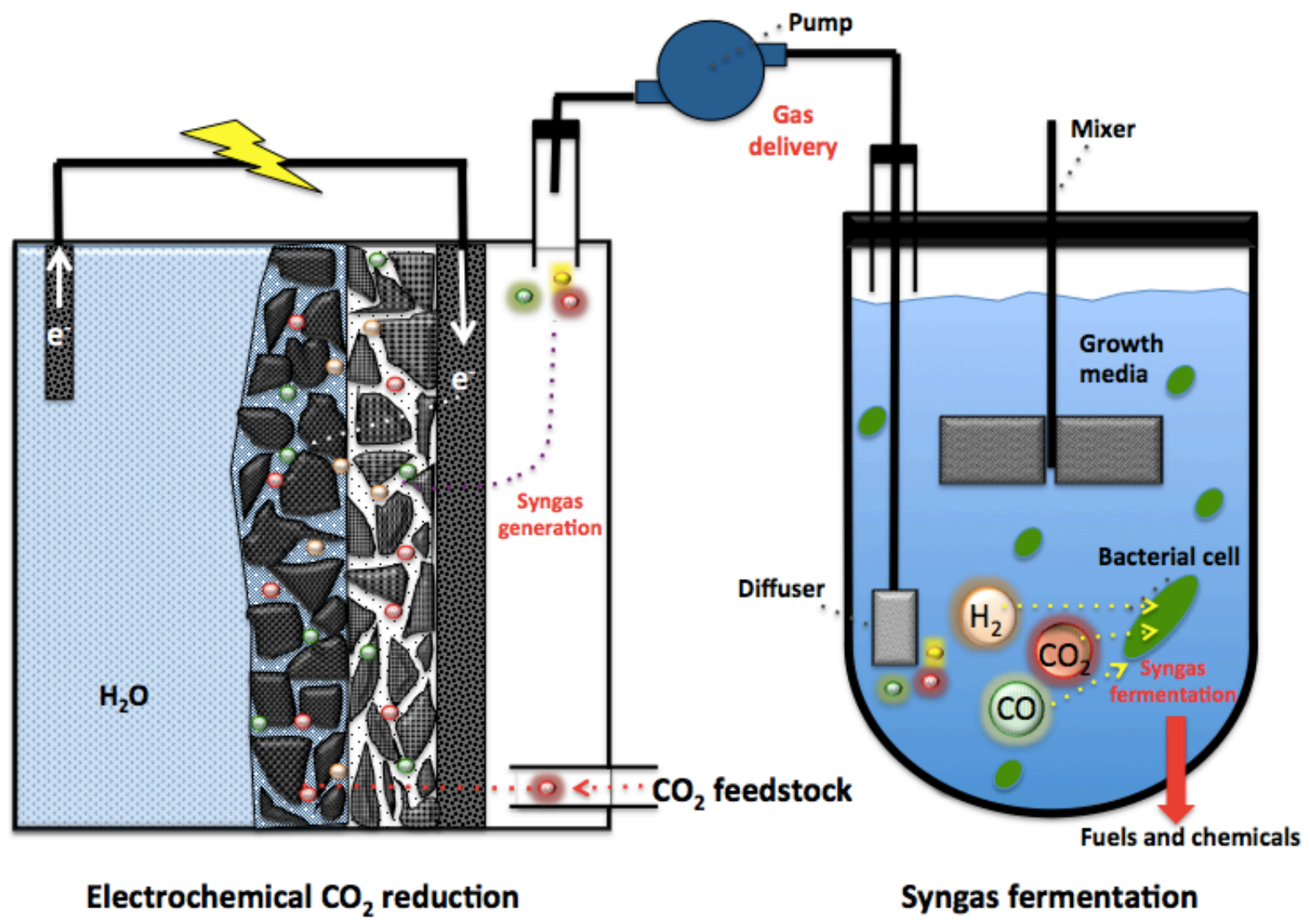

Figure 1: Two-stage biohybrid system for $\mathrm{CO}_{2}$ valorization 


\subsection{Formal Hypothesis}

The following hypotheses can be examined stand-alone but were developed and challenged to investigate the feasibility of creating a biohybrid system that uses renewable energy to power electrochemical $\mathrm{CO}_{2}$ reduction to syngas as an intermediate feedstock for biological conversion to fuels and chemicals.

Hypothesis I. The metabolite generation by Clostridium ljungdhalii can be directed via tuning the $\mathrm{H}_{2} / \mathrm{CO}$ ratio of the syngas feedstock.

Hypothesis II. Fungi grown in wastewater can create a sustainable source of biomass that can be converted to a multi-functional carbon material.

Hypothesis III. $\mathrm{CO}_{2}$ reduction catalysts can be deposited on carbon support material and generate $\mathrm{CO}$ and $\mathrm{H}_{2}$ at high efficiencies. A) Increasing local proton sources of cobalt porphyrins can enhance $\mathrm{CO}_{2}$ reduction capabilities. B) Bulk electrolyte $\mathrm{pH}$ and cation solvation effect $\mathrm{CO}_{2}$ reduction conversion efficiencies

Hypothesis IV. A two-stage biohybrid system can electrochemically reduce $\mathrm{CO}_{2}$ to syngas as an intermediate feedstock for biological conversion into fuels and chemicals via a pure culture of Clostridium ljungdahlii.

\subsection{Dissertation guideline}

This thesis has been designed such that each chapter can be read independently, while also addressing the outlined hypotheses. Chapter 2 tested hypothesis I, that the metabolite generation by Clostridium ljungdhalii can be directed by tuning the $\mathrm{H}_{2} / \mathrm{CO}$ ratio of the syngas feedstock. As $\mathrm{CO}$ and $\mathrm{H}_{2}$ are viable candidates to act as electron mediators, determining their influence on 
product formation was crucial. The results from Chapter 2 were published in Biomass and Bioenergy. Chapter 3 tested hypothesis II, that fungi grown in wastewater can create a sustainable source of biomass that can be converted to a multi-functional carbon material. This hypothesis helped build necessary material synthesis and characterization skills used in the following chapters. The results from Chapter 3 were published in the Journal for Cleaner Production. Chapter 4 investigated hypothesis III, that increasing the cation availability of $\mathrm{CO}_{2}$ reduction catalysts can improve CO production at high efficiencies. Hypothesis IIIa focused on accelerating the electroreduction of $\mathrm{CO}_{2}$ via addition of proton donating ligand groups to cobalt porphyrins. Hypothesis IIIb focused on the effects of bulk electrolyte $\mathrm{pH}$ and solvating ions on $\mathrm{CO}_{2}$ reduction performance. The results will be submitted for publication to the Journal of $\mathrm{CO}_{2}$ Utilization. Chapter 5 tested the hypothesis IV that a two-stage biohybrid system can electrochemically reduce $\mathrm{CO}_{2}$ to syngas as an intermediate feedstock for biological conversion to fuels and chemicals. The results from Chapter 5 are being prepared for submission to Green Chemistry. Future research directions are discussed in Chapter 6. Work describing the integration of platinum nanoparticles attached to covalent organic frameworks for electrochemical hydrogen production is discussed. These results are a portion of a manuscript being submitted to the Journal of American Chemical Society. Moreover, biohybrid research involving the use of functionalized hollow fiber membranes is also presented. 


\section{CHAPTER 2}

\section{DIRECTING CLOSTRIDUM LJUNGDAHLII FERMENTATION VIA $\mathrm{H}_{2} /$ CO SYNGAS RATIO}

\subsection{Introduction}

Synthesis gas (syngas) is a gaseous mixture containing mainly carbon monoxide (CO), hydrogen

$\left(\mathrm{H}_{2}\right)$ and carbon dioxide $\left(\mathrm{CO}_{2}\right)$ generated from the gasification of biomass or other carbon-rich resources such as coal. It has been demonstrated that syngas can be used to generate a variety of valuable chemicals such as acetate, ethanol and 2,3-butanediol (2,3-BD) at high yields and specificities [24]. The low cost and versatility of the syngas feedstock makes it a very attractive approach for chemical production. In general, the conversion of syngas into commodities is performed using either metal-based catalysts (known as Fischer-Tropsch process) or microbial catalysts (known as syngas fermentation). Compared to metal-based catalysis, microbes offer several advantages, such as higher product selectivity, greater product titers, lower energy costs, and greater resistance to poisoning [25-28].

Presently, several anaerobic bacteria have been recognized to grow autotrophically on syngas components. Due to its ability to efficiently utilize both $\mathrm{H}_{2}$ and $\mathrm{CO}$, C. ljungdahlii has been researched as a promising microbial catalyst for syngas fermentation. The unique ability for these microbes to grow autotrophically on these substrates relies on the Wood-Ljungdahl pathway (WLP) that has been reviewed in detail [Fig. A.1; [29-31]]. Briefly, when CO is available it can provide reducing equivalents via $\mathrm{CO}$ oxidation catalyzed by the carbon monoxide dehydrogenase (CODH) enzyme in a biological water-gas shift reaction (Eq. A.1). If $\mathrm{CO}$ is absent, reducing equivalents can be provided by $\mathrm{H}_{2}$ via hydrogenase enzymes (Eq. A.2) and $\mathrm{CO}_{2}$ must be used as the carbon source instead. Both mechanisms (using $\mathrm{CO}$ or $\mathrm{H}_{2} / \mathrm{CO}_{2}$ ) 
ultimately produce acetyl-CoA that can be hydrolyzed to acetate or ethanol. If enough energy is available to the cell, acetyl-CoA can be used to generate pyruvate (Eq. A.3). Pyruvate is a central intermediate for anabolic processes and can lead to the production of more reduced products including 2,3-BD (Fig. A.2).

The microbial conversion of gaseous substrates to products can be viewed macroscopically as a two-step process involving first the transport of the gas to the liquid/cell surface followed by the utilization of the gas by the cell [32]. As a result, bioconversion is in part limited by the physical and thermodynamic attributes of the gaseous substrates available. Examining the properties of the electron donors $\left(\mathrm{H}_{2}\right.$ and $\left.\mathrm{CO}\right)$, it can be noted that they differ greatly in thermodynamic and mass transfer capabilities (Table A.1). For instance, CO delivers electrons at a much lower half-cell potential and releases about $120.18 \mathrm{~kJ} / \mathrm{mol}$ more Gibbs free energy during its conversion to ethanol than $\mathrm{H}_{2}$ (Table A.1; Eq. A.5, [31]). Furthermore, $\mathrm{CO}$ $\left(\mathrm{K}_{\mathrm{H}}=7.4 \mathrm{mM} / \mathrm{atm}\right)$ has a much greater Henry's law constant than $\mathrm{H}_{2}\left(\mathrm{~K}_{\mathrm{H}}=0.78 \mathrm{mM} / \mathrm{atm}\right)$ and thus can more readily partition into the liquid state for cellular uptake [33]. Due to differences in physiochemical properties such as these, the composition of products generated via fermentation is greatly affected by the mixture of the syngas present.

Although the amount of $\mathrm{CO}_{2}$ amongst syngas blends is relatively consistent (typically between $5-15 \%$ ), the relative $\mathrm{H}_{2}$ and $\mathrm{CO}$ concentrations can vary extensively depending on the feedstock, gasifier type, and processing conditions used [34]. The National Energy Technology Laboratory (NETL) recently found $\mathrm{H}_{2}$ and $\mathrm{CO}$ concentrations of coal-derived syngas ranges between 2-34\% and 1-59\%, respectively [35]. As a result, syngas $\mathrm{H}_{2} / \mathrm{CO}$ ratios can range between 0.03-2.0, however, most coal-derived syngas contains $\mathrm{H}_{2} / \mathrm{CO}$ ratios between 0.4-1.0 
[11]. Similarly biomass-derived syngas typically contains $\mathrm{H}_{2} / \mathrm{CO}$ ratios between $0.4-1.0$ but can range between 0.2-4.6 [35].

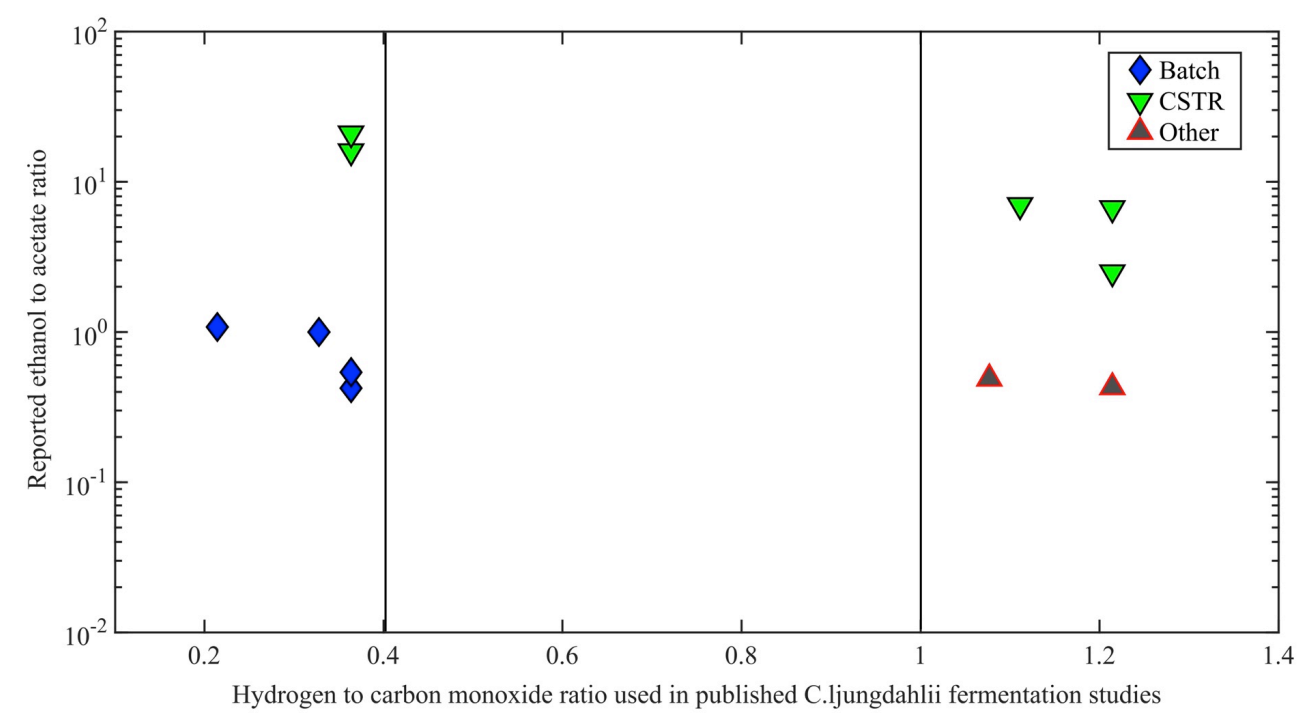

Figure 2: Clostridium ljungdahlii syngas fermentation- Maximum reported ethanol to acetate concentration ratio (log scale) vs. $\mathrm{H}_{2} / \mathrm{CO}$ ratio of the syngas used for culturing [36-46]. Vertical lines indicate boundaries of typical range for syngas $\mathrm{H}_{2} / \mathrm{CO}$ ratios [34-35]. Batch cultures- blue diamonds; continuously stirred tank reactor (CSTR) culturing- downward facing green triangles; other culturing methods (i.e tubular reactor, immobilized cell bioreactor)- red triangles.

Due to the fact that syngas composition is quite variable, research studies that have focused on C. ljungdahlii syngas fermentation have used a wide variety of blends. Comparing published literature it can be seen, in general, when culturing with higher $\mathrm{CO}$ concentrations (relative to $\mathrm{H}_{2}$ ) more electron-dense products are generated (e.g., ethanol) [Fig. 2]. For example, when growing $C$. ljungdahlii in a continuously stirred tank reactor (CSTR) under an $\mathrm{H}_{2} / \mathrm{CO}$ ratio of 0.36 , a maximum ethanol to acetate ratio of 16 was obtained. Conversely, when using a higher $\mathrm{H}_{2} / \mathrm{CO}$ ratio of 1.2 , a maximum ethanol to acetate ratio of only 2.5 was achieved under similar conditions [38,41]. Phillips et al. [32] made similar observations of product formation when comparing the growth of $C$. ljungdahlii on $\mathrm{H}_{2} / \mathrm{CO}_{2}$ vs. $\mathrm{CO} / \mathrm{CO}_{2}$. 
This relationship between syngas and product composition is, however, difficult to predict using present data as for the same $\mathrm{H}_{2} / \mathrm{CO}$ ratio the product ratio can vary across orders of magnitude due to differences in important fermentation parameters such as culturing method, media composition, $\mathrm{pH}$, and gas pressure used across published data. Currently, there is limited understanding on how syngas blends can effect relative product composition. This information could be useful in process design as it can aid in selecting for parameters that produce syngas blends tailored toward desired commodities (i.e. acetate, ethanol, 2,3-BD). Heiskanen et al. [47] acknowledged the importance of syngas compositions on product formation and noted dramatic shifts in acetic and butyric acid production by Butyribacterium methylotrophicum when adding $\mathrm{H}_{2}$ or $\mathrm{CO}_{2}$ to a $\mathrm{CO}$ filled culturing headspace. In the present study, the impact of syngas composition on growth and metabolite production of $C$. ljungdahlii was systematically investigated with the goal of deriving a clear relationship between syngas $\mathrm{H}_{2} / \mathrm{CO}$ ratios and product composition. This was accomplished by culturing microbes with syngas blends across common $\mathrm{H}_{2} / \mathrm{CO}$ ratios and fixing key parameters such as growth media composition, $\mathrm{pH}$, initial gas pressure, and culturing method.

\subsection{Materials and methods}

All microbial cultures were maintained in $162.5 \mathrm{~mL}$ Wheaton serum bottles (Borosilicate glass, Fisher Scientific, UK) containing $50 \mathrm{~mL}$ of ATCC PETC 1754 liquid media [48, 49]. The initial $\mathrm{pH}$ of the media was adjusted to 7.0 using a concentrated $1 \mathrm{M}$ solution of potassium hydroxide [50]. Prior to autoclaving $\left(121^{\circ} \mathrm{C}\right.$ for 30 minutes), ultra high purity argon gas (General Air

Service Supply- UN1006) was diffused into the liquid media for approximately 45 minutes to remove any oxygen. Gas impermeable butyl rubber septum-type stoppers and aluminum crimp 
seals were then used to seal the bottles and the headspaces were purged with argon for another 10 minutes. After autoclaving, the bottles were cooled to room temperature $\left(25^{\circ} \mathrm{C}\right)$.

The headspace pressure of each bottle was then normalized to $0.5 \mathrm{~atm}$ by removal of excess argon gas using sterilized needled syringes and a needled pressure gauge (Geothermalk4517N41600). For all syngas cultures, $150 \mathrm{~mL}$ of filter-sterilized syngas composed of the following $\mathrm{H}_{2} / \mathrm{CO}$ ratios; $0.5,1.0,1.5,2.0$ was then injected directly into the headspace using sterile needled syringes and verified with a gas chromatographer. In a similar manner, $10 \%$ of the final headspace was filled with filter sterilized high purity $\mathrm{CO}_{2}($ General Air Service SupplyUN1013) to create an initial headspace pressure of $1.6 \mathrm{~atm}$ as verified with a sterile needled pressure gauge. The media in each serum bottle were then reduced by addition of $0.5 \mathrm{~mL}$ of the reducing agent to make final concentrations of $3.1 \mathrm{mM} \mathrm{L}$-cysteine- $\mathrm{HCl}$ and $1.7 \mathrm{mM}$ sodium sulfide hydrate.

Fructose was omitted from all syngas test cultures but was used to propagate a seed culture. The seed culture contained $5 \mathrm{~g} / \mathrm{L}$ fructose and was prepared using the same methods as the syngas cultures without the addition of $\mathrm{H}_{2} / \mathrm{CO}$ or removal of argon. Prior to inoculation, the seed culture was grown for 48 hours to an optical density (OD) of 0.8 . After the reducing agent was added to the syngas cultures, $2.5 \mathrm{~mL}$ of the seed culture was used as inoculum. All cultures were incubated horizontally on an orbital shaker incubator, set at $200 \mathrm{rpm}$ and $37^{\circ} \mathrm{C}$.

\subsection{Gas analysis}

Gas samples were obtained directly from the headspace of batch cultures using sterilized gastight syringes in a sterile laminar flow hood. $\mathrm{H}_{2}$ and $\mathrm{CO}$ molar headspace concentrations were determined by manually injecting $250 \mu \mathrm{l}$ of sample into a gas chromatograph (Agilent 
Technologies-7890A) equipped with a thermal conductivity detector (TCD) and packed column [19]. The oven temperature was a constant $60^{\circ} \mathrm{C}$. Injector and detector temperatures were stable at $150^{\circ} \mathrm{C}$ and $250^{\circ} \mathrm{C}$, respectively. Helium gas was used as the carrier gas at a flow rate of $5 \mathrm{~mL}$ $\min ^{-1} . \mathrm{CO}_{2}$ molar headspace concentrations were monitored by manually injecting $100 \mu \mathrm{l}$ of sample into a gas chromatograph (Hewlett Packard-5890) equipped with a thermal conductivity detector (TCD) and packed column [51]. The oven temperature was a constant $60^{\circ} \mathrm{C}$. Injector and detector temperatures were stable at $150^{\circ} \mathrm{C}$ and $190^{\circ} \mathrm{C}$, respectively. Helium gas was used as the carrier gas at a flow rate of $30 \mathrm{~mL} \mathrm{~min}^{-1}$. Molar concentrations were determined by fitting data to a five-point standard curve prepared for each gas.

\subsection{Liquid analysis}

Liquid samples $(1 \mathrm{~mL})$ were withdrawn directly through the top of each culture bottle using sterile needled syringes in a laminar flow hood. The liquid samples were analyzed for optical density $(600 \mathrm{~nm})$ as well as acetate, ethanol, and 2,3-BD content. Optical density measurements were made using a spectrophotometer (Beckman Coulter-DU800) at a fixed wavelength of 600nm. Metabolites were analyzed via high-pressure liquid chromatography [51]. Prior to analysis, liquid samples were passed through $0.22 \mu \mathrm{m}$ filters. Organic acids were separated on an Aminex HPX-87H X column with $4 \mathrm{mM} \mathrm{H}_{2} \mathrm{SO}_{4}$ as the eluent. Molar concentrations were determined by fitting peak areas to a standard curve prepared by diluting ethanol fermentation analytical standard solutions (Bio-Analaytical-LQC-NREL-50) and 2,3BD HPLC standard mixes (Sigma Aldrich-42038).

\subsection{Yield and conversion efficiency computations}


To investigate the bioconversion of gases into metabolites, product yields $\left(\mathrm{Y}_{\mathrm{p} / \mathrm{s}}\right)$ and electron-mol (e-mol) recoveries were calculated for each culturing condition [52]. Yields were determined using Eq. 1 (below):

$$
Y\left(\frac{\text { mol- }-p}{\text { mol-s }}\right)=\frac{\sum_{i=1}^{N} n_{i} \text { produced }}{n_{h_{2} \text { consumed }+n_{\text {co }} \text { consumed }}} \text {, Eq. } 1
$$

where $n_{(i)} n_{h 2}$ and $n_{c o}$ are the number of moles of product $i$, hydrogen and carbon monoxide, respectively. Theoretical yields were determined directly from stoichiometry via Eqs. A.4-A.9 [52].

The efficiency of electrons converted from gas into products was evaluated using Eq. 2 (below)

$$
\mathrm{e}-\text { mol recovery }(\%)=\frac{\sum_{i=1}^{N}\left(n_{(i)} \text { produced } \cdot n_{e^{-}(i)}\right)}{n_{H_{2}} \text { consumed } \cdot n_{e^{-} H_{2}}+n_{\text {Co }} \text { consumed } \cdot n_{e^{-} \text {co }}} \cdot 100, \text { Eq. } 2
$$

where $n_{\left(e^{-}{ }^{-}\right)}$and $n_{\left(e^{-} c o\right)}$ are the number of $\mathrm{e}^{-}$molar equivalents per mol of hydrogen and carbon monoxide.

\section{3 Results and discussion}

\subsection{Syngas consumption under different gas mixture ratios}

C. ljungdahlii effectively utilized both $\mathrm{CO}$ and $\mathrm{H}_{2}$ across the range of $\mathrm{H}_{2} / \mathrm{CO}$ ratios tested. At a given ratio, mass transfer and thermodynamic favorability each played a key role in gas consumption and the products generated. In general, gas consumption rates showed linear relationships with relative gas concentrations likely due to mass transfer limitations of the gases to the liquid/cell surface. For instance, maximum $\mathrm{CO}$ consumption rates in the $\mathrm{H}_{2} / \mathrm{CO}$ ratios range of 0.5 to 1.5 showed a near linear decrease with decreasing initial concentration of $\mathrm{CO}$ [Fig. 3]. Comparable CO consumption rates of ca. $0.45 \mathrm{mM} / \mathrm{hr}$ were observed using $\mathrm{H}_{2} / \mathrm{CO}$ ratios between 1.5 and 2.0. This was likely because CO pressures were low enough for mass transfer to 
not significantly affect consumption rates but instead uptake was limited by reaction kinetics at the cell surface.

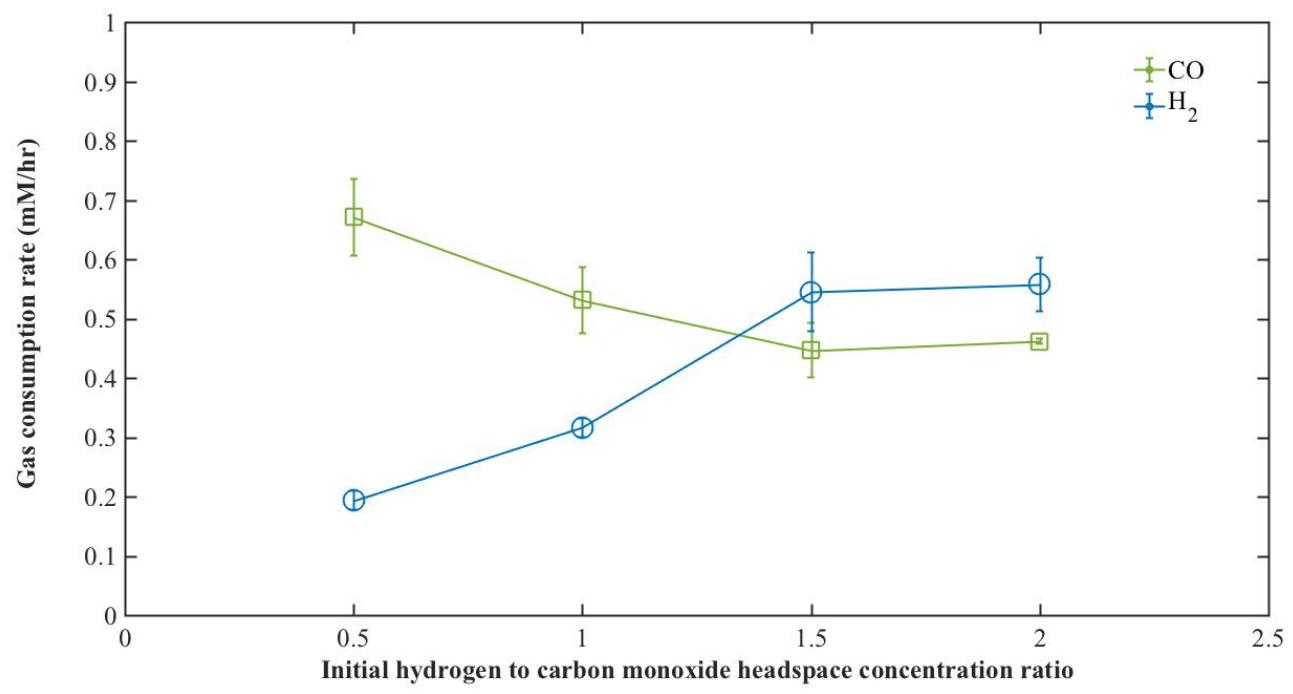

Figure 3: Clostridium ljungdahlii gas consumption rates-Maximum $\mathrm{CO}$ consumption rates (green squares) averaged from duplicate batch cultures with varying initial syngas blends in headspace during first 240 hours of cultivation; Maximum $\mathrm{H}_{2}$ consumption rates (blue circles) averaged from duplicate batch cultures with varying initial syngas blends in headspace during first 240 hours of cultivation; Standard deviation shown in error bars.

Analogous to $\mathrm{CO}$ consumption, $\mathrm{H}_{2}$ utilization also seemed to be limited by gas-liquid mass transfer when using $\mathrm{H}_{2} / \mathrm{CO}$ ratios in the range of 0.5 to 1.5 as a proximate linear trend was observed between $\mathrm{H}_{2}$ consumption rates and the relative concentration of the gas. When using $\mathrm{H}_{2} / \mathrm{CO}$ ratios in the range of 1.5 to 2.0 , comparable $\mathrm{H}_{2}$ consumption rates of ca. $0.55 \mathrm{mM} / \mathrm{hr}$ were observed, indicating that mass transfer was likely no longer limiting at these high concentrations. Furthermore, $\mathrm{H}_{2}$ consumption rates were faster than $\mathrm{CO}$ consumption in this range [Fig. 3].

Thermodynamically $\mathrm{CO}$ is the preferred substrate as its oxidation delivers more Gibbs free energy to the cell than reactions with $\mathrm{H}_{2}$. Comparable utilization of both $\mathrm{H}_{2}$ and $\mathrm{CO}$ only occurred when using an $\mathrm{H}_{2} / \mathrm{CO}$ ratio of 1.5 likely because the higher relative concentration of $\mathrm{H}_{2}$ 
adequately enhanced gas-liquid mass transfer to outcompete the thermodynamic favorability of $\mathrm{CO}$ as a substrate. In contrast, $\mathrm{CO}$ was consumed at greater rates than $\mathrm{H}_{2}$ when using $\mathrm{H}_{2} / \mathrm{CO}$ ratios of 0.5 and 1.0. The affinity for $\mathrm{CO}$ over $\mathrm{H}_{2}$ at these ratios likely stems from a combination of superior gas/liquid mass transfer abilities (larger Henry's law constant) and because more Gibbs free energy is available to the cell when it is used as a substrate.

It is important to recognize that $\mathrm{CO}_{2}$ is both a reactant in the reactions of $\mathrm{H}_{2}$ and a product of $\mathrm{CO}$ reactions. Therefore, the free energy of these reactions and thus the relative affinity of $C$. ljungdahlii for each substrate $\left(\mathrm{H}_{2}\right.$ and $\left.\mathrm{CO}\right)$ are influenced by the concentration of $\mathrm{CO}_{2}$. Figure 4 shows $\mathrm{CO}_{2}$ concentrations after 48,120 , and 240 hours of culturing under each initial $\mathrm{H}_{2} / \mathrm{CO}$ ratio. During the first 48 hours, $\mathrm{CO}_{2}$ was produced in all cultures. This indicates that $\mathrm{CO}$ was being utilized, producing $\mathrm{CO}_{2}$ as a by-product of cell growth.

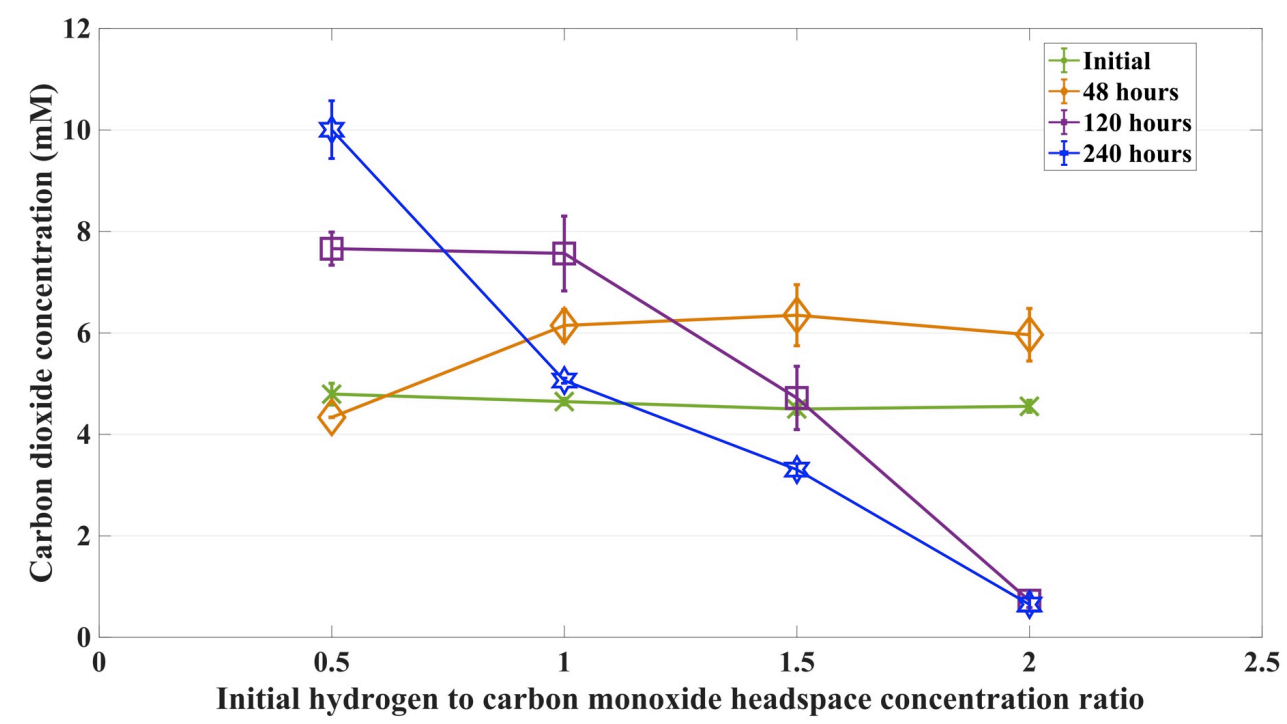

Figure 4: Comparison of Clostridium ljungdahlii $\mathrm{CO}_{2}$ production-Average $\mathrm{CO}_{2}$ concentration of batch cultures with varying initial syngas blends in headspace; Initial values shown as green Xs, concentrations after 48 hours as yellow diamonds; after 120 hours as purple squares; after 240 hours as blue stars; Standard deviation shown in error bars. 
After 120 hours, $\mathrm{CO}$ was fully consumed in $\mathrm{H}_{2} / \mathrm{CO}$ ratios above 1.0 and cultures began to consume $\mathrm{H}_{2}$ with $\mathrm{CO}_{2}$ as the carbon source. This is best exemplified by cultures grown on $\mathrm{H}_{2} / \mathrm{CO}$ ratios of 2.0 that consumed more than $5 \mathrm{mM}$ of $\mathrm{CO}_{2}$ during the $48-120$ hour period of cultivation. As $\mathrm{H}_{2}$ consumption continued concurrently with $\mathrm{CO}_{2}$ fixation, a near linear trend developed after 240 hours of culturing between $\mathrm{CO}_{2}$ concentrations and initial headspace $\mathrm{H}_{2}$ content. Specifically, cultures with higher initial $\mathrm{H}_{2} / \mathrm{CO}$ ratios $(\geq 1.5)$ ultimately consumed $\mathrm{CO}_{2}$ to final concentrations lower than the initial concentration, while cultures with lower initial $\mathrm{H}_{2} / \mathrm{CO}$ ratios $(<1.5)$ produced $\mathrm{CO}_{2}$ to final concentrations greater than the initial [Fig. 4]. This is likely because at higher $\mathrm{H}_{2} / \mathrm{CO}$ ratios, more hydrogen is consumed than $\mathrm{CO}$, increasing the demand for $\mathrm{CO}_{2}$ as a carbon source.

\subsection{Product generation under different gas mixture ratios}

C. ljungdahlii synthesized chemicals directly from syngas components. A spectrum of products was generated dependent on the relative concentration of each gaseous substrate. Acetate and ethanol were the major products produced under all initial $\mathrm{H}_{2} / \mathrm{CO}$ ratios tested. Figure 5 shows the maximum product concentrations achieved during cultivation using each initial $\mathrm{H}_{2} / \mathrm{CO}$ ratio. Acetate formation was favored by increased $\mathrm{H}_{2}$ concentrations in the headspace with a maximum acetate concentration of $35.21 \pm 0.84 \mathrm{mM}$ achieved using an $\mathrm{H}_{2} / \mathrm{CO}$ ratio of 2.0 . Acetate production gradually dropped along with decreasing $\mathrm{H}_{2} / \mathrm{CO}$ ratios as the lowest concentration $(22.64 \pm 0.55 \mathrm{mM})$ was obtained at a ratio of 0.5 . This trend is in agreement with previous work

that shows increased $\mathrm{H}_{2}$ concentrations lead to increased acetate formation in pure and mixed consortia of carboxydotrophs $[53,54]$. 


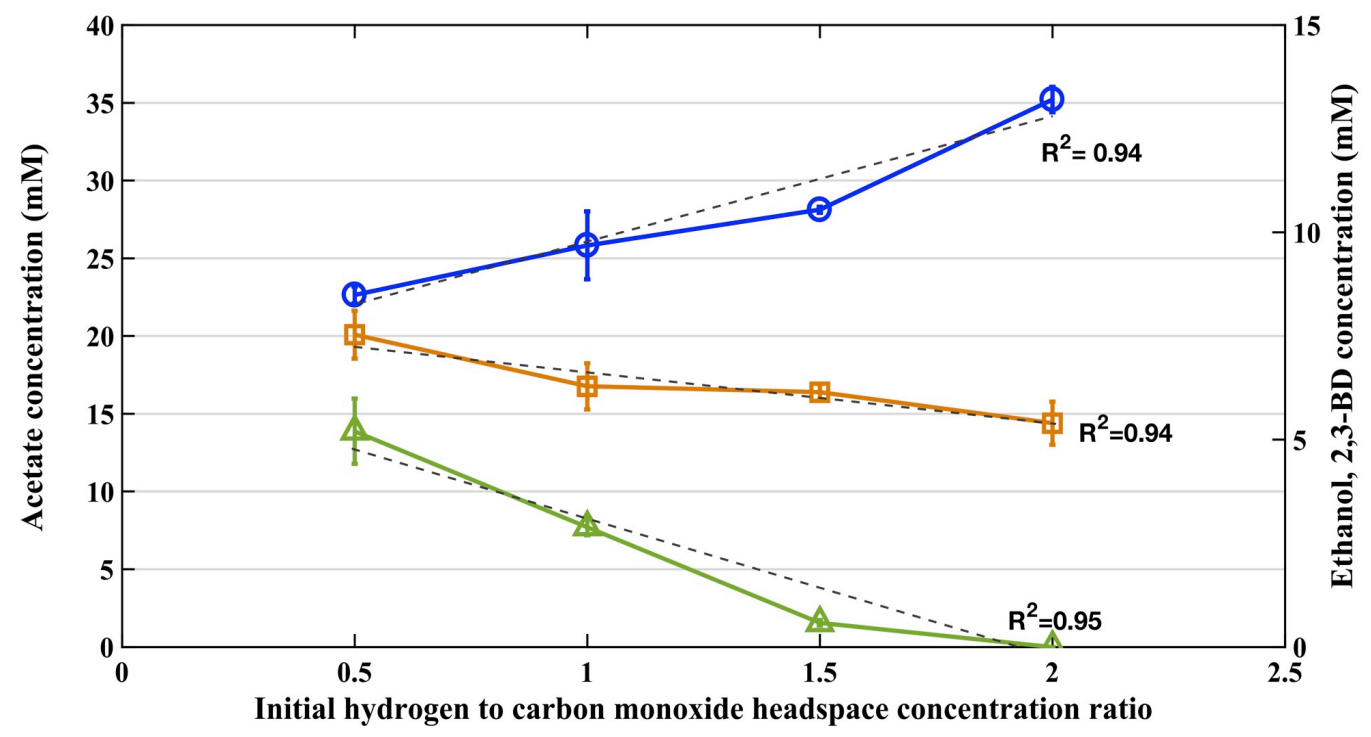

Figure 5: Impact of $\mathrm{H}_{2} / \mathrm{CO}$ ratio on product formation- Maximum concentration of acetate, ethanol, and 2,3BD achieved during first 240 hours of cultivation under each initial $\mathrm{H}_{2} / \mathrm{CO}$ ratio tested; Acetate-blue circle; Ethanol- orange square; 2,3-BD- green triangle; Averaged from duplicate batch cultures; Linear regression shown as dashed lines with coefficients of determination; Standard deviation shown in error bars.

In contrast, the production of more reduced metabolites was favored by increased concentrations

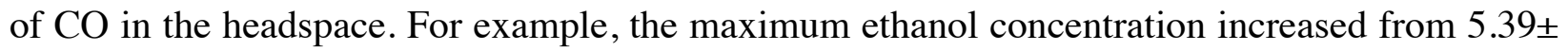
$0.52 \mathrm{mM}$ to $7.44 \pm 0.67 \mathrm{mM}$ when the $\mathrm{H}_{2} / \mathrm{CO}$ ratios decreased from 2.0 to 0.5 . Similarly, the production of 2,3-BD was also favored by increased $\mathrm{CO}$ in the headspace reaching a maximum concentration of $5.20 \pm 0.79 \mathrm{mM}$ using an $\mathrm{H}_{2} / \mathrm{CO}$ ratio of 0.5 . Interestingly, almost no 2,3BD was formed under the highest $\mathrm{H}_{2}$ concentrations (i.e., using $\mathrm{H}_{2} / \mathrm{CO}$ ratios of 1.5-2.0), suggesting that the additional free energy from CO reactions was necessary for the formation of this more reduced metabolite.

Complementary to acetate formation, a near linear relationship between $\mathrm{H}_{2} / \mathrm{CO}$ ratio and both ethanol and 2,3-BD production was observed across the range of $\mathrm{H}_{2} / \mathrm{CO}$ ratios tested [Fig. 5]. This relationship between product formation and syngas compositions can be expected 
thermodynamically. The higher Gibbs free energy released with $\mathrm{CO}$ reactions stems from the ability of $\mathrm{CO}$ to directly become the carbonyl moiety in the eastern branch of the WLP. When $\mathrm{CO}$ is absent, $\mathrm{CO}_{2}$ has to be reduced to $\mathrm{CO}$ consuming additional electrons (from $\mathrm{H}_{2}$ ), yielding less free energy and electron-dense by products. This phenomenon is exemplified in Figure 5 that shows the lowest ethanol concentration of $5.39 \pm 0.52 \mathrm{mM}$ occurred under the highest $\mathrm{H}_{2}$ concentration.

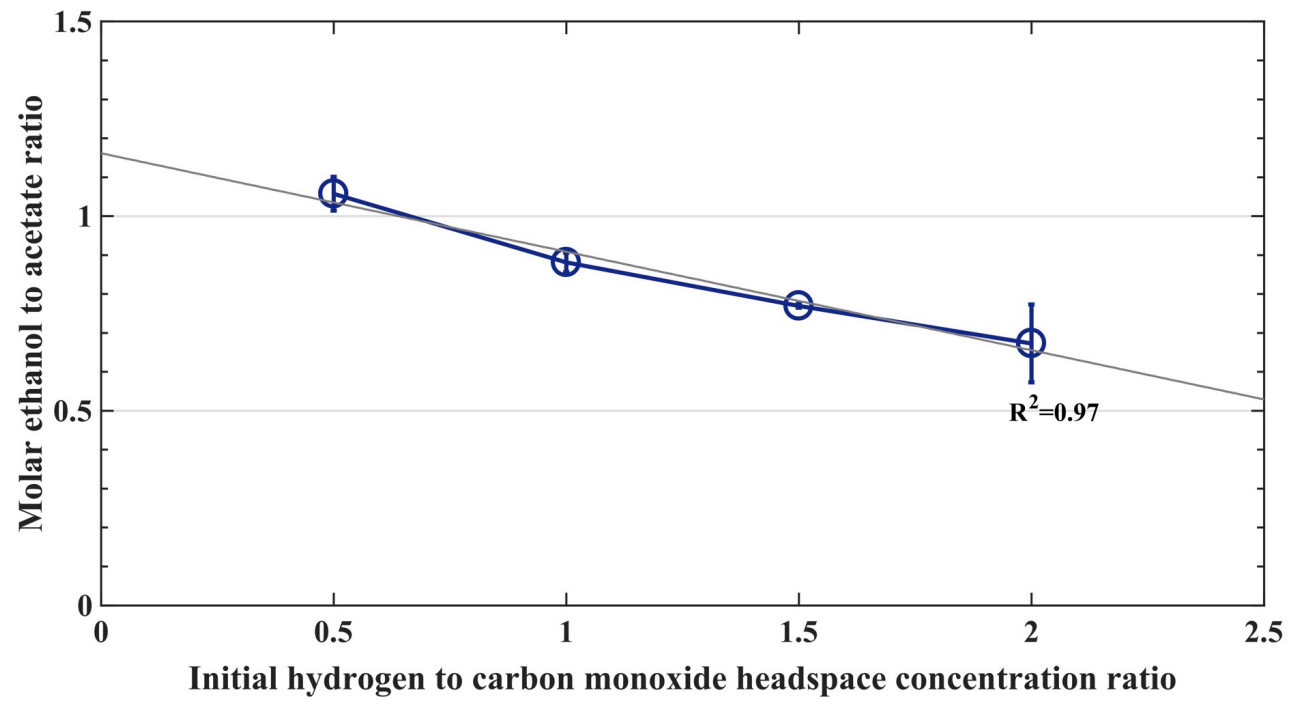

Figure 6: Comparison of Clostridium ljungdahlii acetate and ethanol production -Maximum molar acetate to ethanol ratios observed under varying initial syngas blends in headspace; Averaged from duplicate batch cultures; Linear regression shown as dashed line; Standard deviation shown in error bars.

This relationship is further demonstrated by the proximate linear trend obtained from plotting the maximum ethanol to acetate ratio achieved by each culture vs. the initial $\mathrm{H}_{2} / \mathrm{CO}$ ratio [Fig. 6]. As expected, the greatest ethanol to acetate ratio of $1.06 \pm 0.04$ was observed under the highest $\mathrm{CO}$ concentrations (i.e. using an $\mathrm{H}_{2} / \mathrm{CO}$ ratio of 0.5 ).

\subsection{Clostridium ljungdahlii growth on different syngas mixtures}


C. ljungdahlii effectively grew across the range of $\mathrm{H}_{2} / \mathrm{CO}$ ratios tested. All cultures grew within 48 hours of cultivation, indicated by increases in optical density. Figure 7 shows optical densities of liquid samples measured by a spectrophotometer at a fixed wavelength of $600 \mathrm{~nm}$ after 48 , 120 , and 240 hours of cultivation under each initial $\mathrm{H}_{2} / \mathrm{CO}$ ratio tested.

Thermodynamically, it would be expected that anabolic processes would be favored by greater levels of $\mathrm{CO}$ as more free energy is available to the cell when it is used as the electron donor. Cell growth is, however, dictated by ATP content. When $\mathrm{CO}$ is used as the primary electron donor, more products such ethanol and 2,3-BD are generated, at the expense of ATP production. For example, when CO is utilized via the WLP an investment of one ATP is required in the western branch to convert formate into formyltetrahydrofolate (CHO-THF) [Fig. A.1]. CHO-THF is eventually used to generate acetyl-CoA, the building block for products such as acetate, ethanol and pyruvate. Since CO utilization results in the generation of more reduced products, less ATP/electrons is available for cell growth than when $\mathrm{H}_{2}$ is the primary electron donor. A linear trend between cell growth and increasing initial $\mathrm{H}_{2}$ content is then expected. This was observed in this study as after 48-120 hours of cultivation, growth was favored by increased $\mathrm{H}_{2}$ in the headspace. 


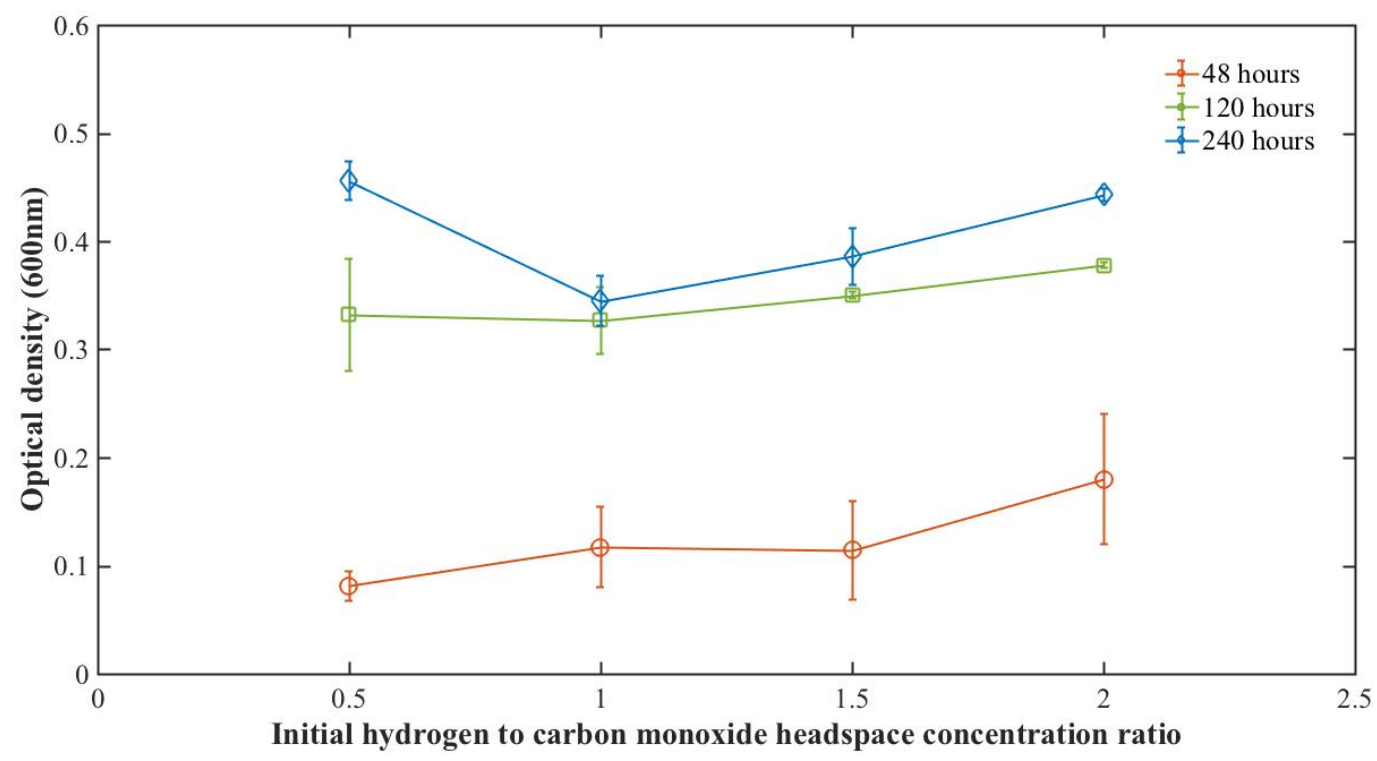

Figure 7: Clostridium ljungdahlii growth on syngas- Average optical density of batch cultures with varying initial syngas blends in headspace; After $48 \mathrm{hrs}$ of cultivation- orange circle; After $120 \mathrm{hrs}$ of cultivation-green square; After $240 \mathrm{hrs}$ of cultivations-blue diamond; Averaged from duplicate cultures; Standard deviation shown as error bar

Interestingly, cultures in the $\mathrm{H}_{2} / \mathrm{CO}$ range of 1.0-1.5 grew slightly less than others after 240 hours. This phenomenon may be partly attributed to the unique chemical properties of CO. Although, $\mathrm{CO}$ is an excellent metabolite for its ability to serve as a carbon source and provide electrons at an extremely low potential, it can also potentially disrupt other cellular operations $[30,55]$. CO contains a triple bond that makes it a weak Lewis base and an unsaturated soft ligand that can accept metal $\mathrm{d} \pi$ electrons. As a result, $\mathrm{CO}$ acts as both a pi acceptor and sigma donor that can form stable complexes with the low-valent states of the metal sites in enzymes. This property makes $\mathrm{CO}$ a strong inhibitor of metalloproteins that use low valent metals in their operations, such as hydrogenases that function to bind diatomic gases (i.e. $\mathrm{H}_{2}$ ) [30].

Cultures in the $\mathrm{H}_{2} / \mathrm{CO}$ range of 1.0-1.5 may have grew slightly less than others due to minor inhibition of their hydrogenase enzymes via high concentrations of $\mathrm{CO}$. At an $\mathrm{H}_{2} / \mathrm{CO}$ ratio 
of 2.0 there was likely not enough $\mathrm{CO}$ to cause notable inhibition of hydrogen uptake as the highest optical density of ca. 0.45 was achieved after 240 hours [Fig. 7]. Similar optical densities were also achieved at $\mathrm{H}_{2} / \mathrm{CO}$ ratios of 0.5 . Although this may be explained by experimental error, it is likely that since $\mathrm{CO}$ was the dominant substrate any inhibition of hydrogen may have been too minimal to cause observable difference in cell growth.

\subsection{Clostridium ljungdahlii product yields and efficiencies}

Product yields and e-mol recoveries were calculated to further evaluate the bioconversion of gases into metabolites under each culturing condition [Fig. 8]. The highest yields of $0.188 \pm .01$ mol-p/mol-s and 0.203 \pm .02 mol-p/mol-s were achieved under syngas ratios of 0.5 and 2.0, respectively, while cultures under ratios of 1.0 and 1.5 achieved similar yields ca. 0.161 molp/mol-s. All experimental yield values were comparable to those determined in similar C.ljungdahlii batch fermentation studies [56].

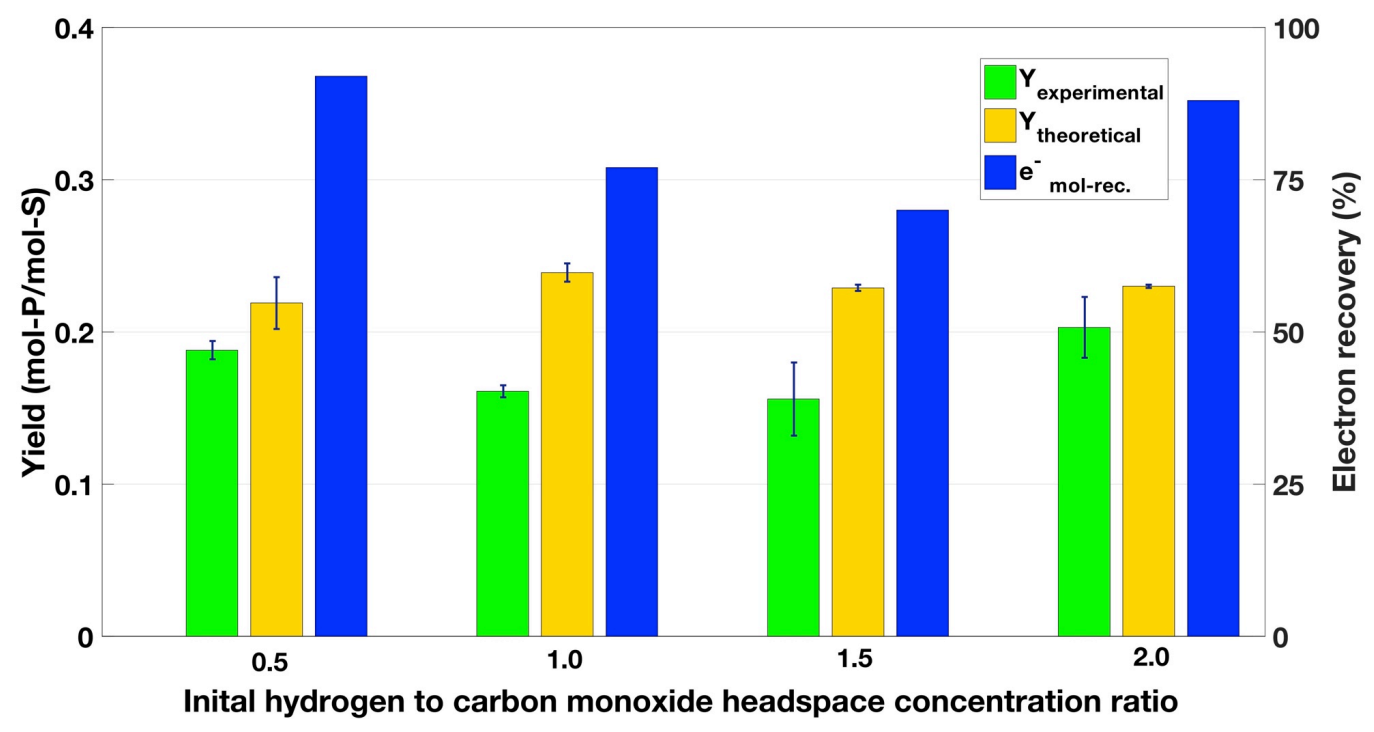


Figure 8: Clostridium ljungdahlii product yield and efficiencies; Experimental and theoretical yields: mol of products per mol of gas consumed (left y-axis); total electron-mol recovered in products (right y-axis)

As expected, product yields followed a similar trend as cell growth. It is likely that the generation of metabolites by 1.0 and 1.5 were partly limited by $\mathrm{CO}$ inhibition of hydrogenase enzymes as noted by others [57]. This was likely the cause of lower yields and electron recovery in products (70-77\%). Interestingly, the yields of cultures under the 0.5 and 2.0 syngas blends were similar, despite having significantly different mixtures of products. This is also reflected in that both culturing scenarios achieved ca. $87 \%$ of theoretical yield values. These findings are parallel to those of Gaddy et al. [32] when culturing C.ljungdahlii on $\mathrm{H}_{2} / \mathrm{CO}_{2}$ and $\mathrm{CO} / \mathrm{CO}_{2}$. Analogous to these results, e-mol recoveries for cultures using 0.5 and 2.0 syngas blends were in a similar range between $88-92 \%$. It is expected that the remaining electrons were used to generate other minor metabolites or were needed to fulfill additional energy requirements for cell maintenance $[43,58]$. Notably, the composition of the metabolites generated was dependent on the syngas blend used although conversion efficiencies were comparable under each culturing scenario.

\subsection{Conclusions}

The results of this study demonstrate that the $\mathrm{H}_{2} / \mathrm{CO}$ ratios of syngas play a critical role in the spectrum of products generated via syngas fermentation by $C$. ljungdahlii. Production of electron-dense products was favored by $\mathrm{CO}$ in the headspace. Linear relationships were obtained that describe the impact of the syngas $\mathrm{H}_{2} / \mathrm{CO}$ ratios in the range of $0.5-2.0$ on acetate, ethanol, and 2,3-BD formation, respectively. This information on the relative formation of products in relation to $\mathrm{H}_{2} / \mathrm{CO}$ ratios will be useful in selecting syngas production parameters that can now be 
more accurately optimized for selecting syngas composition tailored toward desired product generation. 


\section{CHAPTER 3}

\section{PRODUCTION OF MAGNETIC BIOCHAR FROM WASTE-DERIVED FUNGAL BIOMASS FOR PHOSPHORUS REMOVAL AND RECOVERY}

\subsection{Introduction}

Phosphorus is an essential element for life in aquatic ecosystems, however, excess amounts introduced from agricultural runoff or municipal wastewater can have extremely harmful effects such as eutrophication of receiving waters [59]. As such, development of efficient and sustainable methods that remove phosphorus from aqueous systems are needed to protect wildlife and meet increasingly rigid pollution regulations worldwide. To date, various techniques involving biological treatment, membrane separation, chemical precipitation, electrolysis and adsorption have been investigated $[60,61]$. Of these technologies, phosphorus adsorption onto biochar is considered to be one of the most promising due to it's low cost, high selectively, ecofriendliness, and ease of operation $[62,63]$.

Michalekova- Richveisova et al. (2017) recently demonstrated maximum phosphate adsorption capacities of $0.036 \mathrm{mg} / \mathrm{g}, 0.132 \mathrm{mg} / \mathrm{g}$, and $0.296 \mathrm{mg} / \mathrm{g}$ using corn-cob, garden wood waste, and wood chips biochars, respectively [64]. Others, however, have demonstrated far better phosphorus adsorption capabilities using biochars modified with iron particles. For example, Cai et al. (2016) reported a maximum phosphate adsorption of $5.07 \mathrm{mg} / \mathrm{g}$ using magnetic biochar derived from water hyacinth [65]. Notably, the modification of biochars with iron does not only make biochar a better adsorbent for some contaminates but also holds the added benefits of easy removal and recovery using simple magnets [66].

Though beneficial, the production of most magnetic biochars can be quite complicated and unsustainable. The majority of current methods involve a set of secondary processes in which a 
magnetic medium is deposited on the surface of a char $[67,68]$. For instance, Wang et al. (2015) prepared magnetic pine wood biochar by mineralizing hematite and mixing the suspension with the feedstock material prior to pyrolysis [69]. Alternatively, Yang et al. (2018) impregnated iron directly into waste-activated sludge biomass prior to pyrolysis and found the phosphorus adsorption capabilities were greatly improved compared to samples fabricated using deposition methods [70]. Nevertheless, current methods such as these involve the use of expensive chemicals, complicated processing techniques, and generation of complex wastes.

In this work, we present a simple and novel bottom-up method for magnetic biochar production that alleviates these drawbacks and demonstrates excellent performance. Unique to our method, iron precursors are not impregnated in the char nor deposited onto the biochar postpyrolysis, but rather accumulated and encapsulated within the living biomass feedstock prior to processing. The key to this innovative approach is the use of widely available municipal waste streams in combination with the robust fungus, Neurospora crassa (N. crassa), to generate biomass as the feedstock material for pyrolysis. N. crassa is capable of bio-accumulating high concentrations of many metals such as iron, cobalt, and nickel at high yields without affecting biomass growth [71-73]. Furthermore, N. crassa is easy to culture and exhibits a rapid growth rate on many minimal media. As such, N.crassa has been used in a wide variety of biological studies and its genome has been thoroughly mapped making it amenable to genetic manipulation [74]. The culmination of these unique qualities allow for a cheap, sustainable and adaptable source of biomass.

N.crassa is grown directly in iron-rich wastewater streams, which not only enables magnetic biochar production but also accomplishes wastewater treatment by removing metals and organics 
from the waste stream. Typical waste streams can be from water treatment plants that use iron coagulants and primary effluent from wastewater treatment or industrial wastewater streams.

Here we demonstrated for the first time that N. crassa wild type could be grown on blends of waste streams, and the resulting biomass could be effectively processed into a magnetic biochar material. We further demonstrate that the physiochemical properties of the materials could be tuned by controlling the relative volumes of each of the waste streams used in the growth media. This technique allows for a low-cost, versatile, and sustainable approach to create magnetic biochar directly from municipal waste. The structure and morphology of the processed chars were investigated using a suite of tools including scanning electron microscopy equipped with an energy-dispersive X-ray analyzer (SEM-EDS), BET-N $\mathrm{N}_{2}$ adsorption, X-ray photoelectron spectroscopy (XPS), X-ray powdered diffraction (XRD) and Fourier transform spectroscopy (FTIR). To confirm the adsorbent properties of these materials, batch phosphate adsorption studies were conducted. Adsorption mechanisms were analyzed by fitting experimental data to Langmuir, Freundlich and Temkin isotherms and characterizing the P-loaded chars.

\subsection{Materials and methods}

\subsubsection{Material production and processing}

$N$. crassa was grown in waste streams consisting of primary effluent (PE) from Boulder wastewater treatment facility (Boulder, $\mathrm{CO}$ ) and iron coagulation backwash (BW) from Boulder reservoir water treatment plant (Boulder, CO). Due to low organic content, BW alone was not able to sustain biomass growth so it was diluted with PE at ratios of 1:4 (PE-BW 1:4) or 3:4 (PEBW 3:4). Prior to use, both waste streams were autoclaved at $121^{\circ} \mathrm{C}$ for $15 \mathrm{~min}$. Glucose was then blended with waste streams at $1 \%(\mathrm{w} / \mathrm{v})$ to stimulate the fungal acclimation. The major 
constituents of each growth blend prior to fungal inoculation were determined by inductively coupled plasma mass spectrometry (Perkin Elmer SCIEX-ICP-MS; Fig. A.3).

$N$. crassa wild- type strain (FGSC \#262) stock was stored on Vogel's minimal media slants at $-20{ }^{\circ} \mathrm{C}[75]$. New cultures were initiated by growing cells on agar slants at $30{ }^{\circ} \mathrm{C}$ for 3 days in complete darkness. Conidia (fungal spores) were isolated from slants using standard methods and inoculated into $1 \mathrm{~L}$ flasks of media at a 1:100 dilution [76]. After inoculation, fungal cultures were stored on an orbital incubator/shaker (Barnstead Max Q4000) at $30^{\circ} \mathrm{C}$ and $90 \mathrm{rpm}$ for 48 hours. The resulting biomass was then harvested using a vacuum pump and 0.45 $\mu \mathrm{m}$ filters and dried in a desiccator for 24 hours at $50^{\circ} \mathrm{C}$. The dry biomass was carbonized by heating the biomass to $700^{\circ} \mathrm{C}$ at a rate of $10^{\circ} \mathrm{C} / \mathrm{min}$ of using an isotemp muffle furnace (Fischer Scientific-550 series). The resulting granular fungal char was then processed into a powder using a ball mill for 20 minutes (Retsch-PM100). It is expected that the particle size after grinding is less than 325 meshes. Figure 9 summarizes the fabrication method of the magnetic biochar. 


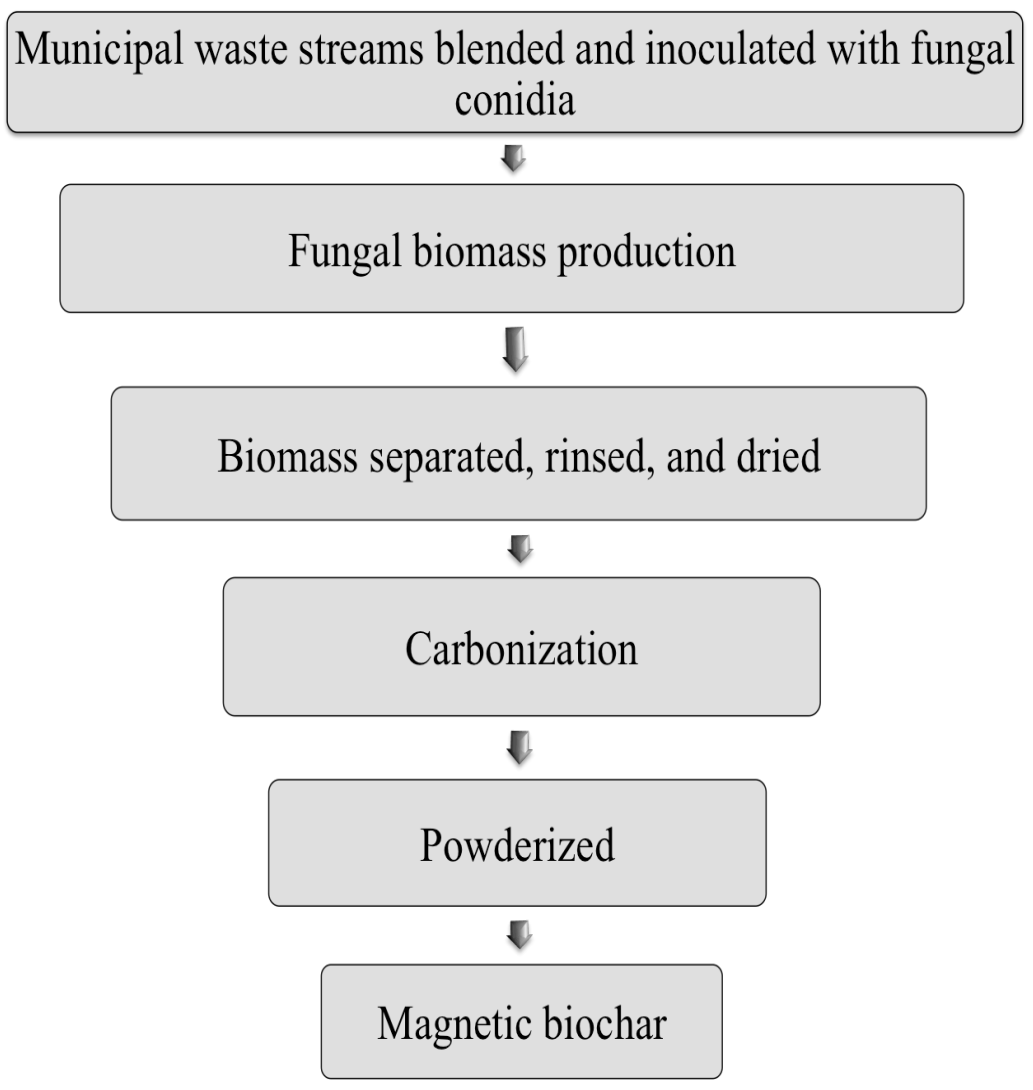

Figure 9: Flow diagram for magnetic biochar production- Fungal biomass derived from waste streams processed into powdered magnetic biochar material. 1) Growth media prepared by blending waste-streams and inoculating fungal spores. 2) Fungal cultures grown for 48 hours at $37^{\circ} \mathrm{C}$, agitated at $90 \mathrm{rpm}$. 3) Biomass filtered using $0.45 \mu \mathrm{m}$ filter then dried in a desiccator at 50 ${ }^{\circ} \mathrm{C}$ for 24 hours 4) Dry biomass carbonized in a muffle furnace at $700{ }^{\circ} \mathrm{C}$ 5) Granular material processed to a powder using a ball mill.

\subsubsection{Material characterization}

The magnetic properties of the chars were determined using a simple magnet. To test the stability of the magnetic properties, both chars were put into aqueous solution (pure water; $0.1 \mathrm{~g} / \mathrm{L}$ ) and rotated at $120 \mathrm{rpm}$ for 14 days at $25{ }^{\circ} \mathrm{C}$ in the dark. The morphology and structure of the powdered biochars were investigated using SEM (Jeol JSM- 6480LV, $90 \mathrm{kV}$ ). The elemental composition of the materials were examined using EDS (Oxford Instruments Link ISIS) coupled to the SEM. Surface composition was further investigated using FTIR spectra recorded in the 
$4000-400 \mathrm{~cm}^{-1}$ region with a resolution of $4 \mathrm{~cm}$ (Bruker Vector 22 FTIR spectrometer). Further analysis of the materials surfaces were conducted using X-ray photoelectron spectroscopy (PE5800 X-ray Photoelectron Spectroscopy) preformed in the range of $0-1100 \mathrm{eV}$. The surface crystallinity of each material was analyzed to identify Fe-bearing minerals using an X-ray diffractometer (XRD; Rigaku Homelab) equipped with a stepping motor, a graphite crystal monochromator, and a CuKa radiation source. The total surface area, average pore size, and total pore volume were measured using $\mathrm{N}_{2}$ sorption on a NOVA 1200 analyzer and calculated using the BET method.

\subsubsection{Batch adsorption experiments and kinetic testing}

Batch adsorption experiments were conducted in $55 \mathrm{~mL}$ polypropylene tubes using $100 \mathrm{mg}$ of adsorbent and $50 \mathrm{~mL}$ phosphorus solutions prepared with sodium phosphate (Sigma Aldrich342483). Initial phosphorus concentrations of 4, 8, 16, 50, 70 and $90 \mathrm{mg}-\mathrm{P} / \mathrm{L}$ were tested. As determined by preliminary experiments, adsorption equilibrium was achieved after shaking the tubes for 24 hours in a rotary shaker $(150 \mathrm{rpm})$ at room temperature $\left(25 \pm 2{ }^{\circ} \mathrm{C}\right)$ in the dark. The solutions were then centrifuged at $4000 \mathrm{rpm}$ for $15 \mathrm{~min}$. The supernatant was sampled using sterile syringes and passed through $0.22 \mu \mathrm{m}$ filters. All adsorption experiments were run in duplicate. Blank solutions, without adsorbent, were used as controls. Phosphorus concentrations of the absorption solution supernatant were obtained using Hach TNT 844 or High range Molybdovanadate testing kits per the Hach method. Phosphorus loaded chars were collected from centrifuge tubes and allowed to dry for 24 hours at room temperature in the dark. The Ploaded chars were then analyzed using FTIR and EDS.

\subsubsection{Phosphorus adsorption isotherm}


The amount of adsorbed phosphorus per unit biochar mass was calculated using Equation 3 (below).

$$
q_{e}=V\left(C_{0}-C_{e}\right) / m,(\text { Eq. 3) }
$$

where $\mathrm{qe}$ is the equilibrium adsorbent capacity, $\mathrm{C}_{0}$ is the initial and $\mathrm{C}_{\mathrm{e}}$ is the equilibrium concentration of the phosphorous $(\mathrm{mg} / \mathrm{L}) ; \mathrm{V}$ is the volume of the solution $(\mathrm{L}) ; \mathrm{m}$ is the weight of the adsorbent $(\mathrm{g})$

To better understand the underlying reaction mechanisms, data was fit to three adsorption isotherm models (below).

$$
\begin{gathered}
\mathrm{q}_{\mathrm{e}}=\frac{\mathrm{Q}_{\mathrm{o}} \mathrm{K}_{\mathrm{L}} \mathrm{C}_{\mathrm{e}}}{1}+\mathrm{K}_{\mathrm{L}} \mathrm{C}_{\mathrm{e}}, \text { Langmuir (Eq. 4) } \\
\mathrm{q}_{\mathrm{e}}=\mathrm{K}_{\mathrm{f}} \mathrm{C}_{\mathrm{e}}^{\frac{1}{\mathrm{n}}}, \text { Freundlich (Eq. 5) } \\
\mathrm{q}_{\mathrm{e}}=\left(\mathrm{RT} \ln \mathrm{A}_{\mathrm{T}} / \mathrm{b}_{\mathrm{t}}\right)+\left(\mathrm{RT}_{\left.\ln \mathrm{C}_{\mathrm{e}} / \mathrm{b}_{\mathrm{t}}\right), \text { Temkin (Eq. 6) }}\right.
\end{gathered}
$$

where: $\mathrm{q}_{\mathrm{e}}=$ amount of phosphorus adsorbed per gram of the adsorbent at equilibrium $(\mathrm{mg} / \mathrm{g}) ; \mathrm{C}_{\mathrm{e}}=$ the equilibrium concentration of adsorbate $(\mathrm{mg} / \mathrm{L}) ; \mathrm{Q}_{\mathrm{o}}=$ maximum monolayer coverage capacity $(\mathrm{mg} / \mathrm{g}) ; \mathrm{K}_{\mathrm{L}}=$ Langmuir isotherm constant $(\mathrm{L} / \mathrm{mg}) ; \mathrm{K}_{\mathrm{f}}=$ Freundlich isotherm constant $(\mathrm{mg} / \mathrm{g}) ; \mathrm{n}=$ adsorption intensity; $\mathrm{A}_{\mathrm{T}}=$ Temkin isotherm equilibrium binding $(\mathrm{L} / \mathrm{g})$ constant; $\mathrm{b}_{\mathrm{T}}=$ Temkin isotherm constant; $\mathrm{R}=$ universal gas constant $(8.314 \mathrm{~J} / \mathrm{mol} / \mathrm{K}) ; \mathrm{T}=$ Temperature $(\mathrm{K})$

To examine the fit of these models, commonly used adsorption error analysis parameters: sum square of errors (ERRSQ) and average relative error (ARE) were calculated using Eq. 7 and Eq. 8 [77]. ERRSQ is said to be one of the most widely used error functions, however, it has a major disadvantage that at higher end liquid-phase adsorbate concentrations it will illustrate a better fit for experimental data [78]. To mitigate bias, the ARE function which aims at minimizing the fractional error distribution across the entire concentration range was used in conjunction with ERRSQ [79].

$$
E R R S Q=\sum_{i=1}^{n}\left(q_{e, i, c a l c}-q_{e, i, m e a s}\right)^{2}, \text { Sum square of errors (Eq. 7) }
$$




$$
A R E=\frac{100}{n} \sum_{i=1}^{n} \frac{q_{e, i, c a l c}-q_{e, i, m e a s}}{q_{e, i, m e a s}}, \text { Average relative error (Eq. 8) }
$$

where $\mathrm{q}_{\mathrm{e}, \mathrm{i} \text {,meas }}$ is the experimentally measured adsorbed solid phase concentration of the absorbate on the absorbent as determined using Eq.3 and $\mathrm{q}_{\mathrm{e}, \mathrm{i}, \text {,calc }}$ is the theoretical concentration of adsorbate on the adsorbent, which has been calculated using one of the isotherm models (i.e. Eq. 4, Eq. 5, or Eq.6).

\subsection{Results and discussion}

\subsubsection{Fungal growth and nutrient uptake}

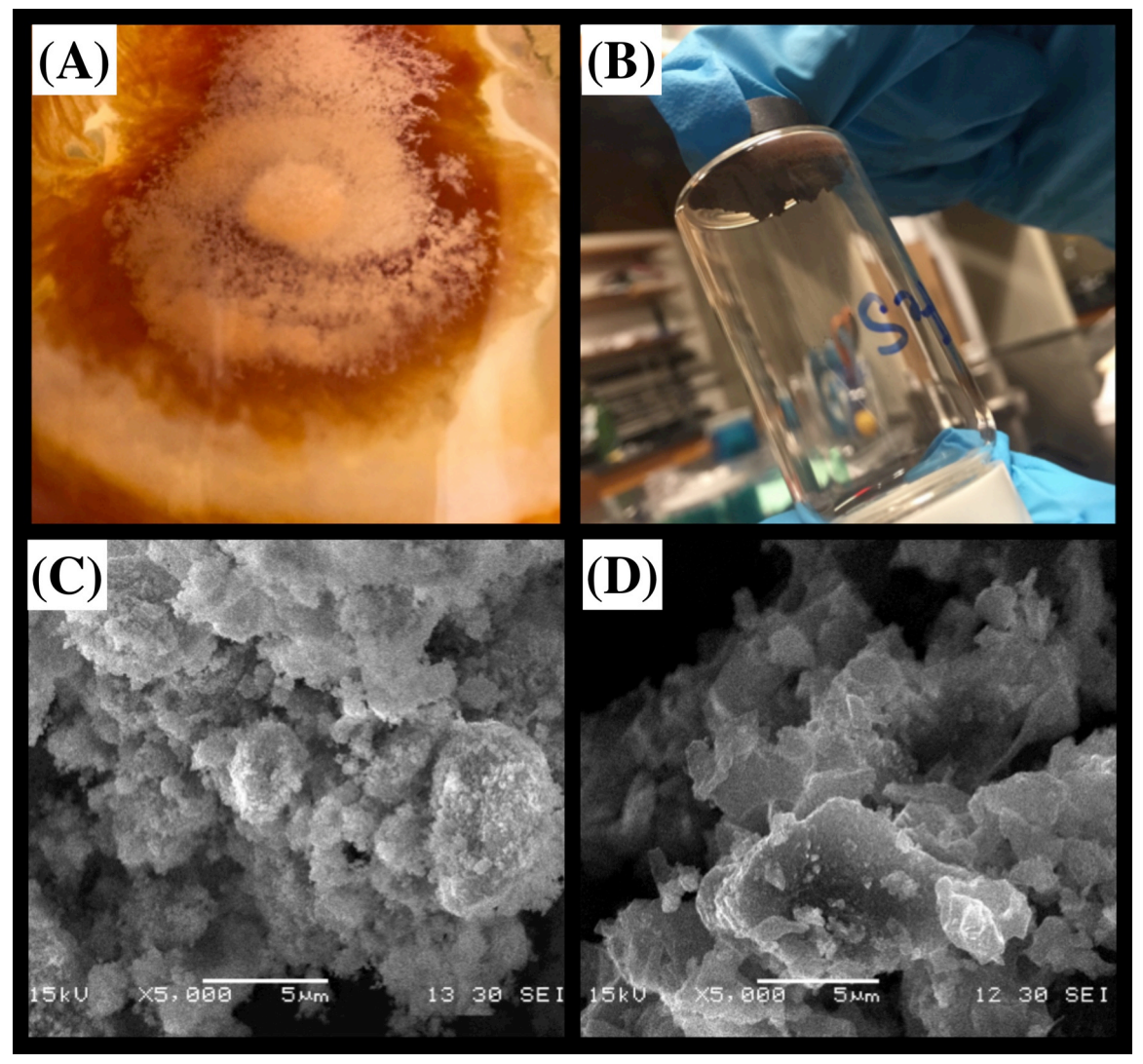

Figure 10: Biochar production and characterization images- Fungal cultures grown for 48 hours at $37^{\circ} \mathrm{C}$, agitated at $90 \mathrm{rpm}$ (A) Researcher demonstrating magnetic abilities of PE-BW 1:4 (B) SEM image (5000x magnification, 5 $\mu \mathrm{m}$ scale bar) of PE-BW 1:4 (C) and PE-BW 3:4 (D)

N.crassa was successfully cultivated on both of the wastewater blends tested. Mature cultures were observed after 48 hours of incubation, indicated by the appearance of conidia (fungal spores) across the surface of the fungal mats [Fig. 10a]. N.crassa depleted large amounts of 
metal from the growth solutions likely via bio-sorption and metabolic uptake pathways as reviewed by others $[80,81]$. Larger biomass yields were attained by fungi grown on the PE-BW 1:4 media due to greater accumulation of metal [Fig. 11]. ICP-MS analysis of the growth media showed fungi grown under the PE-BW 1:4 media consumed $601.34 \mathrm{mg} / \mathrm{L}$ iron, while fungi under the PE-BW 3:4 media consumed only $228.4 \mathrm{mg} / \mathrm{L}$ [Fig. 11].

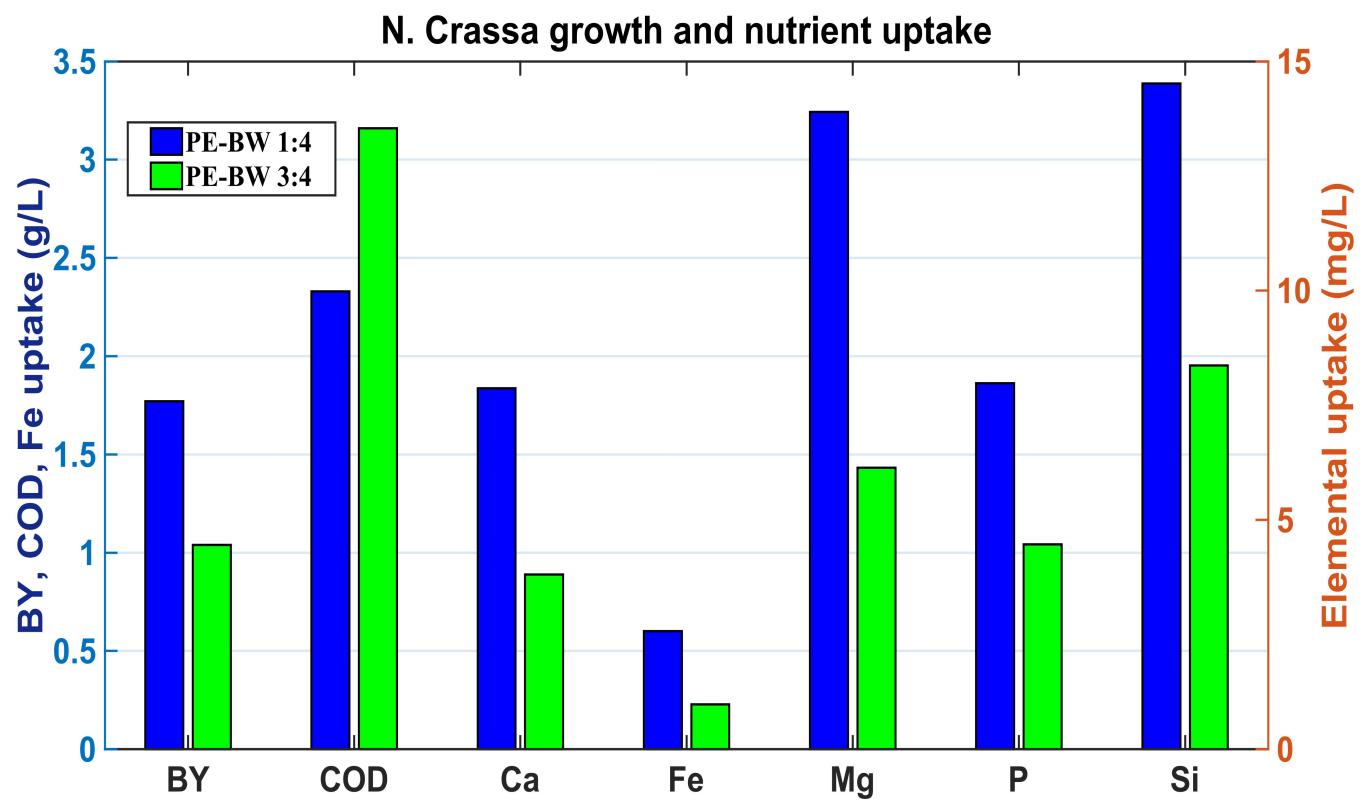

Figure 11: N.Crassa growth and nutrient uptake-Biomass yield (BY), COD removal and iron uptake per liter of wastewater used (left y-axis); $\mathrm{Ca}, \mathrm{Mg}, \mathrm{P}, \mathrm{Si}$ uptake per liter of wastewater media used (right y-axis)

In contrast, carbon uptake was favored by fungi grown on the 3:4 dilution (PE-BW 3:4) that consumed about $0.83 \mathrm{~g} \mathrm{COD} / \mathrm{L}$ more than those under the 1:4 ratio (PE-BW 1:4), likely due to the increased availability of supporting nutrients (i.e. nitrogen, phosphorus, etc.). Other constituents that were readily removed from solution during fungal growth included $\mathrm{Si}, \mathrm{Mg}, \mathrm{P}$ and Ca [Fig. 11]. These nutrients were likely used to augment the utilization of iron or were biomineralized in separate metabolic processes as examined by others [82]. The average dry weight 
of biomass per liter of media used were $1.77 \mathrm{~g}$ for PE-BW 1:4 media and 1.04 $\mathrm{g}$ for PE-BW 3:4 media, respectively [Fig. 11].

\subsubsection{Material characterization}

Fungal biomass resulting from the PE-BW 1:4 and PE-BW 3:4 growth medias were ultimately processed into fungal chars PE-BW 1:4 and PE-BW 3:4. Both finished chars exhibited magnetic properties. PE-BW 1:4 demonstrated a very strong affinity for magnets while PE-BW 3:4 displayed limited attraction [Fig. 10b]. After 14 days of being agitated and submerged in aqueous solution (pure water; $0.1 \mathrm{~g} / \mathrm{L}$ ), both chars retained their magnetic properties and were easily recovered from solution using a simple magnet. SEM images of the biochars revealed morphologies unique to each of the materials [Fig. 10c,d]. The greater initial metal content of the blend used to generate PE-BW 1:4 led to increased crystalline formation during the pyrolysis process compared to PE-BW 3:4. EDS coupled to the SEM revealed distinct elemental composition of each material surface.

Only a very small amount of elemental carbon (2.1\%) was detected in PE-BW 1:4 while PE-BW 3:4 was composed of 23.7\% [Table 1]. Both materials retained similar amounts of iron (ca. 45-50\%), however, PE-BW 1:4 had a much greater Fe/C ratio (21.4 vs. 2.1) and higher oxygen content (19\% vs. 25\%) than PE-BW 3:4 which suggested superior iron oxide formation on the material surface.

Table 1: Major elemental constituents of powdered chars (by weight \%), BET- $\mathrm{N}_{2}$ specific surface area (SA), average pore diameter (APD) and total pore volume (TPV) of biochars.

\begin{tabular}{|l|l|l|l|l|l|l|l|l|}
\hline Material & C (\%) & O (\%) & Si (\%) & Fe (\%) & Fe/C & SA (m $/ \mathbf{g})$ & APD (nm) & TPV (cm $/ \mathbf{g})$ \\
\hline PE-BW 1:4 & 2.1 & 23.9 & 29.8 & 45.0 & 21.4 & 51.6 & 13.2 & 0.2 \\
\hline
\end{tabular}




\begin{tabular}{|l|l|l|l|l|l|l|l|l|}
\hline PE-BW 3:4 & 23.7 & 19.6 & 1.4 & 50.5 & 2.13 & 53.0 & 6.1 & 0.1 \\
\hline
\end{tabular}

This is line with PE-BW 1:4's enhanced magnetic properties compared to PE-BW 3:4 and the crystal arrangements observed in SEM images. The smaller surface area $\left(51.6 \mathrm{~m}^{2} / \mathrm{g}\right)$ and greater average pore diameter $(13.2 \mathrm{~nm})$ of PE-BW 1:4 further proposed amplified iron oxide development as these formations have small surface areas and ample transitional pores (2-50nm). PE-BW 1:4 had about double the total pore volume $\left(0.2 \mathrm{~cm}^{3} / \mathrm{g}\right)$ of PE-BW $3: 4\left(0.1 \mathrm{~cm}^{3} / \mathrm{g}\right)$ even though the carbon content of PE-BW 3:4 was much greater.

This may also be attributed to the superior iron oxide formation of PE-BW 1:4 but could also be due to large silica oxide developments in PE-BW 3:4 as implied by its much greater surface silica content.

To further investigate the crystalline structures, FTIR spectra, XPS and XRD patterns were obtained for each of the powdered chars [Fig. 12]. XRD analyses identified maghemite $(\gamma-$ $\left.\mathrm{Fe}_{2} \mathrm{O}_{3}\right)$ as the major crystalline in PE-BW 1:4 as characterized by strong diffraction peaks at $30.2^{\circ}, 35.5^{\circ}, 43.2^{\circ}, 57.3^{\circ}$, and $62.9^{\circ}[83,84]$. The presence of maghemite was likely the source of magnetic attraction as proposed in similar work [85]. As expected, PE-BW 3:4 showed much weaker maghemite diffraction peaks and presented patterns more similar to an amorphous carbon material. Similar observations were made from the XPS Fe 2p3/2 spectra that showed two peaks at 710.6 and $724.1 \mathrm{eV}$, corresponding to $\mathrm{Fe} 2 \mathrm{p} 3 / 2$ and $\mathrm{Fe} 2 \mathrm{p} 1 / 2$, that are the characteristic peaks of the $\mathrm{Fe}^{3+}$ ion in maghemite. Furthermore, satellite peaks at $719 \mathrm{eV}$ also indicate maghemite formation. In agreement with XRD data, each of the maghemite XPS peaks for PE-BW 1:4 were much stronger than PE-BW 3:4 indicating a greater development of maghemite. 

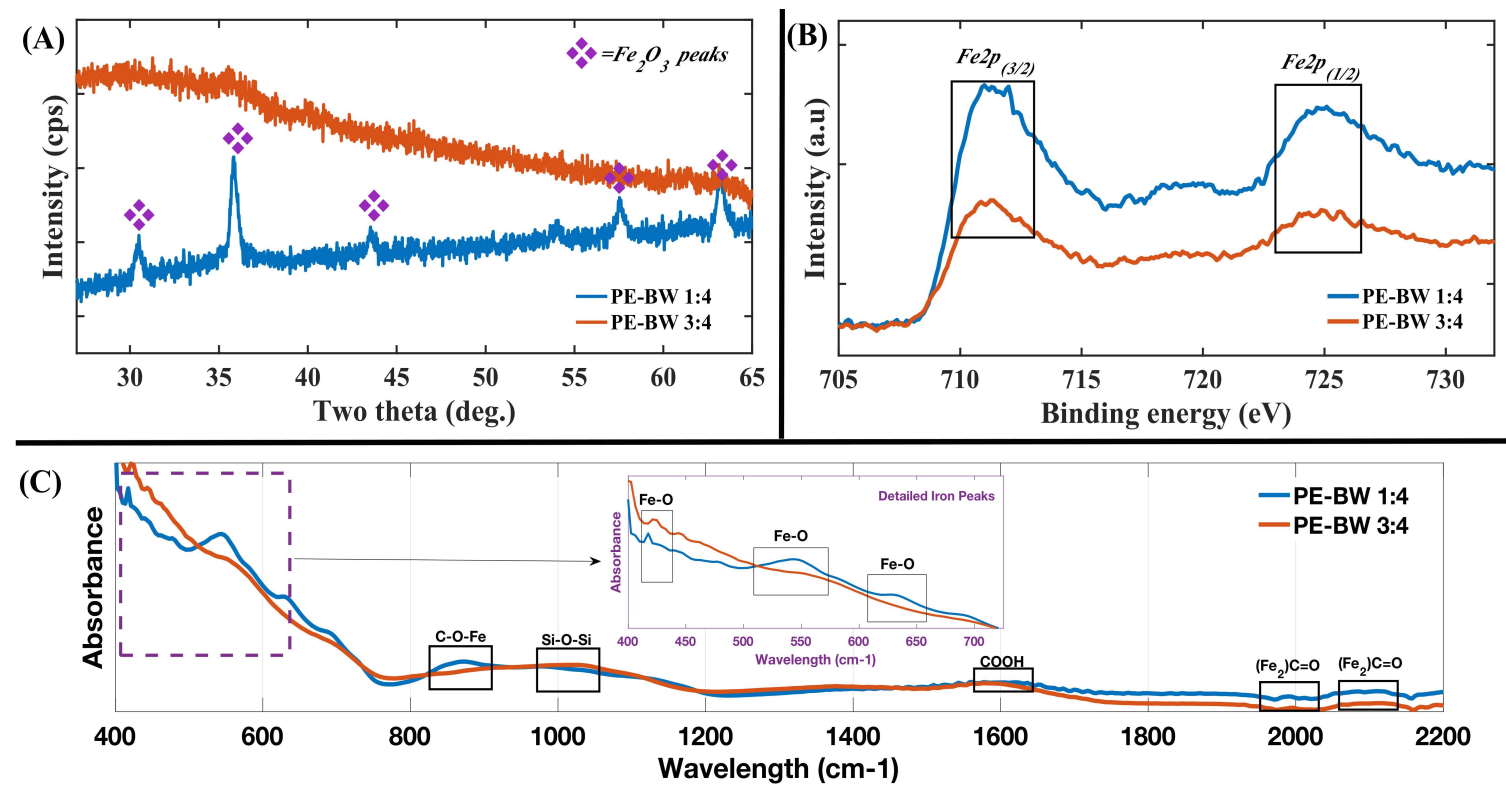

Figure 12: Fungal biochar material characterization summary- XRD diffraction pattern of PEBW1:4, PE-BW 3:4; v indicates maghemitite peaks (A) Zoomed XPS spectra of PE-BW 1:4, PE-BW3:4 (B) FTIR spectra of PE-BW1:4 and PE-BW3:4; zoomed inset of Fe-O peaks (400$725 \mathrm{~cm}^{-1}$; all other peaks of interest boxed and labeled (C)

FTIR spectra recorded from 400 to $4000 \mathrm{~cm}^{-1}$ showed both materials exhibited peaks centered at 432,556 , and $634 \mathrm{~cm}^{-1}$ that suggest the presence of $\mathrm{Fe}-\mathrm{O}$ bonds in the form of maghemitite [86-88]. As expected, PE-BW 1:4 displayed much more pronounced bands than PEBW 3:4 owing to its greater iron oxide formation. PE-BW 1:4 also showed strong peaks around $831 \mathrm{~cm}^{-1}$ that indicate $\mathrm{C}-\mathrm{O}-\mathrm{Fe}$ surface bonds while PE-BW 3:4 displayed broader peaks centered at $1060 \mathrm{~cm}^{-1}$ that suggested silica oxide formation in the form of silicone (Si-O-Si). Both materials retained some organic structures as signified by the presence of transitional metal carbonyl groups with peaks centered at $1974 \mathrm{~cm}^{-1}$ and $2100 \mathrm{~cm}^{-1}$ and broad carboxylate peaks centered at $1600 \mathrm{~cm}^{-1}[67,89]$.

\subsubsection{Phosphorus adsorption isotherms}

Both biochars exhibited strong affinities for phosphorus across the range of initial concentrations tested. The highest equilibrium adsorption density of $23.9 \mathrm{mg} / \mathrm{g}$ was obtained using PE-BW1:4 
and an initial phosphate concentration of $90 \mathrm{mg} / \mathrm{L}$ [Fig. 13]. This high performance demonstrates the practical use of this material in agricultural and environmental applications. For example, municipal wastewaters commonly contain $5-20 \mathrm{mg} / \mathrm{L}$ total phosphorus [90]. In this range, PEBW 1:4 achieved an adsorption density of $2.42 \mathrm{mg} / \mathrm{g}$ using an initial phosphate concentration of $16 \mathrm{mg} / \mathrm{L}$. In comparable studies, Huggins et al. (2016) reported an adsorption capacity for phosphate of $1.0 \mathrm{mg} / \mathrm{g}$ under an initial phosphate concentration of $18 \mathrm{mg} / \mathrm{L}$ using a granular biochar prepared from lodgepole pine wood [60]. Moreover, Chen et al. (2011) reported an adsorption capacity of $0.44 \mathrm{mg} / \mathrm{g}$ under a under an initial phosphate concentration $12 \mathrm{mg} / \mathrm{L}$ using a magnetic biochar orange peel and magnetite deposition [67].

Others have reviewed the affinity for iron particles to complex phosphorus in detail $[67,91]$. It is likely the greater phosphorus adsorption ability of PE-BW1:4 stems from its superior maghemtite development. Moreover, the higher average pore diameter (13.2nm vs. 6.1nm) of PE-BW 1:4 may have further augmented surface interactions with the phosphorus in solution compared to PE-BW 3:4.

To better understand the underlying sorption behaviors of the phosphorus to the chars, data was fitted to the Freundlich, Temkin, and Langmuir isotherm models. All of the models reproduced the isotherm data fairly well with coefficients of determination $\left(\mathrm{R}^{2}\right)$ above 0.90 [Fig. 13]. Both PE-BW 1:4 and PE-BW 3:4 fit the Freundlich adsorption isotherm the best. This is best demonstrated by PE:BW 1:4 which exhibited an $\mathrm{R}^{2}$ value of 0.99 and very low ERRSQ and ARE errors of 33.6 and 35.6, respectively. 

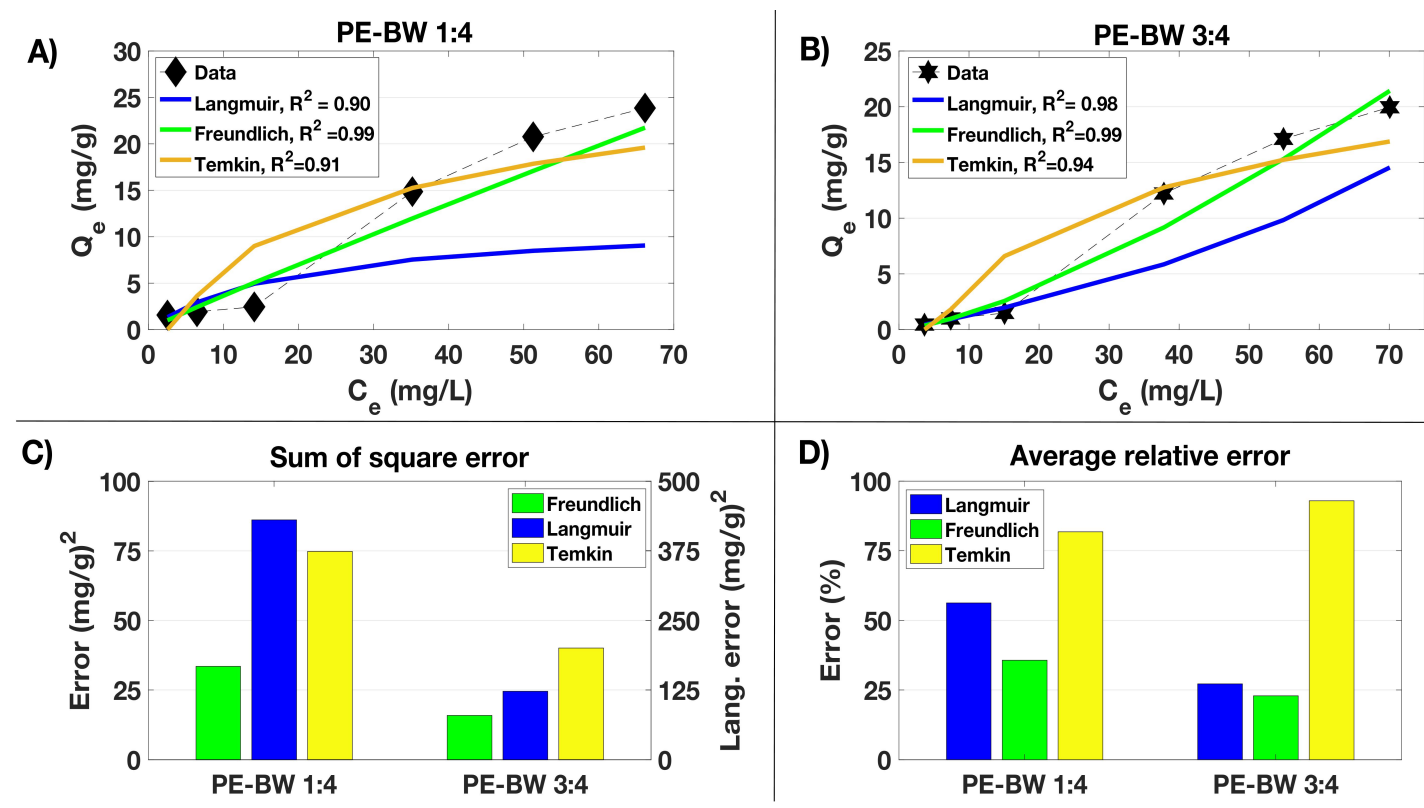

Figure 13: Adsorption isotherms and error analysis of phosphorus on fungi derived biocharsPE-BW 1:4 adsorption isotherms; $\mathrm{u}=$ measured data (A) PE-BW 3:4 adsorption isotherms; $\star$ =measured data (B) Results of sum of square error analysis; Freundlich and Temkin error (left $y-$ axis), Langmuir (Lang.) error (right y-axis) (C) Results of average relative error analysis for each isotherm fit to measured data (D)

The Freundlich model is often used to describe chemisorption on heterogeneous surfaces, which is in line with the composite nature of these materials and likely why the data fit this isotherm the best. This was also found by Lü et al. (2013) in similar studies using Fe-Al-Mn trimetal oxides for phosphate removal [92]. Interestingly, the Temkin model which is also commonly used for heterogeneous surfaces was the worst fit for both PE-BW 1:4 and PE-BW 3:4 with $\mathrm{R}^{2}$ values of 0.91 and 0.94 and large ARE values of 81.2 and 92.9, respectively [Fig. 13]. The Temkin model assumes that adsorption is characterized by a uniform distribution of binding energies, which makes it better suited for homogenous materials than the Freundlich model [93]. In comparison, it may be that the greater homogeny of PE-BW 3:4, caused by less extensive iron oxide surface formation, made it a better fit to the Temkin model than PE-BW 1:4. This was further suggested by fitting the data to the Langmuir model that assumes that adsorption occurs at specific homogeneous sites on the adsorbent. PE-BW 3:4 fit the Langmuir 
model almost as well as the Freundlich model with an $\mathrm{R}^{2}$ of 0.98 , ERRSQ of 122.7 and ARE of 27.2 [Fig. 13]. PE-BW 3:4 fit the Langmuir model much better than PE-BW 1:4, further indicating that its distinct adsorption mechanisms stem from its greater homogeny.

\subsubsection{Phosphate retention and surface complexes}

To validate the phosphorus recovery capabilities of these materials, EDS and FTIR analyses were conducted on the chars post-adsorption [Fig. A.4; Fig. 14]. These analyses have been effectively used in many adsorption studies to elucidate interaction mechanisms between adsorbates and adsorbents $[94,95]$. At grander scale, EDS confirmed the presence of absorbed phosphorus on the chars. For example, after adsorption with a initial phosphate concentration of $50 \mathrm{mg} / \mathrm{L}$ the phosphorus surface atomic percent and weight percent of PE-BW 1:4 rose by $1.52 \%$ and $1.76 \%$, respectively. This is complemented by FTIR analyses that showed noteworthy changes even after adsorption with initial concentrations as low as $10 \mathrm{mg} / \mathrm{L}$ [Fig. 14].

A)

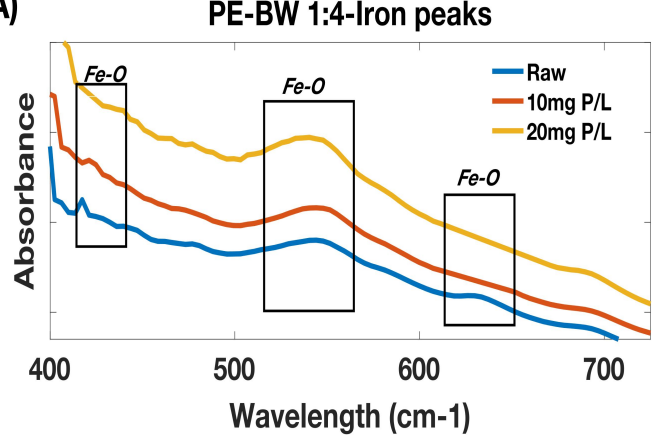

B)

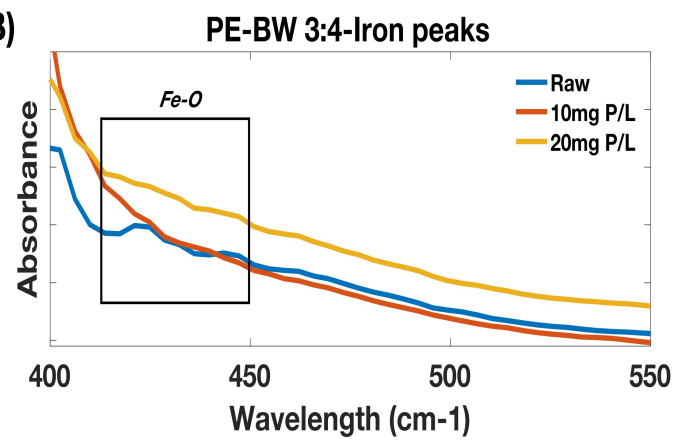

PE-BW 1:4-Phosphorus peaks

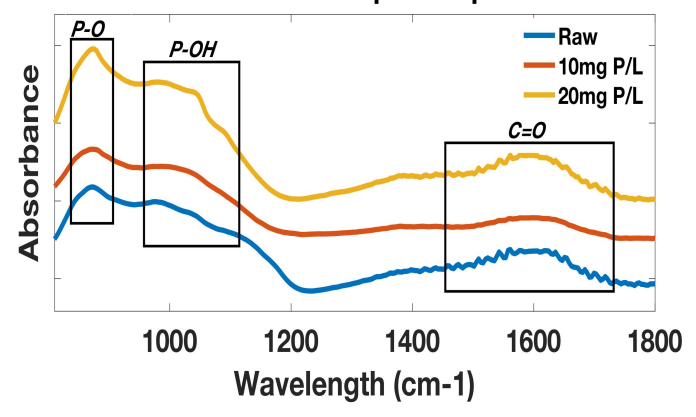

PE-BW 3:4-Phosphorus peaks

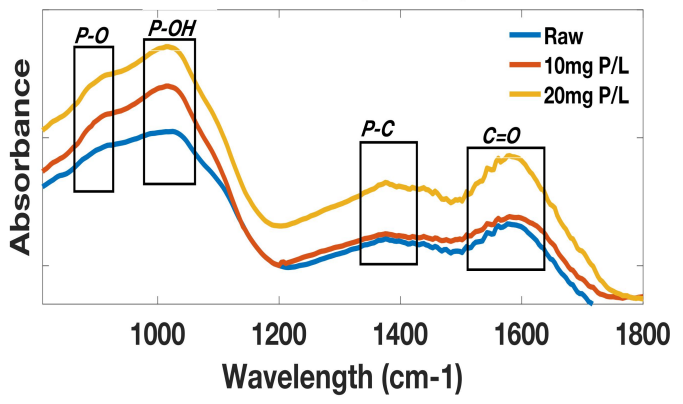


Figure 14: Phosphorus loaded biochar FTIR analysis summary- PE-BW 1:4 iron peaks (left plot) and phosphorus peaks (right plot) for raw material and after equilibrium in batch study with $10 \mathrm{mg} \mathrm{P} / \mathrm{L}$ and $20 \mathrm{mg} \mathrm{P} / \mathrm{L}$ (A) PE-BW 3:4 iron peaks (left plot) and phosphorus peaks (right plot) for raw material and after equilibrium in batch study with $10 \mathrm{mg} \mathrm{P} / \mathrm{L}$ and $20 \mathrm{mg} \mathrm{P} / \mathrm{L}$ (B)

For both materials, the Fe-O bands centered at $442 \mathrm{~cm}^{-1}$ and $634 \mathrm{~cm}^{-1}$ seem to lessen and even disappear post adsorption. Conversely, new bands at $865 \mathrm{~cm}^{-1}$ and $1025 \mathrm{~cm}^{-1}$ appeared after adsorption likely owing to new $\mathrm{P}-\mathrm{O}$ and $\mathrm{P}-\mathrm{OH}$ bonds, similar to findings of others $[91,92]$. The combined results imply a substitution reaction between Fe-O groups with adsorbed P-ions. Interestingly, PE-BW 3:4 also showed the sharpening of a peak around $1380 \mathrm{~cm}^{-1}$ suggesting P-C bonds [Fig. 14]. This may demonstrate a second binding mode that is more pronounced in PEBW 3:4 than PE-BW 1:4 which is expected because PE-BW 1:4 contains much less carbon (2.1\% vs. $23.7 \%)$. Furthermore, both materials showed elevated peaks around $1600 \mathrm{~cm}^{-1}$ which are likely linked to $\mathrm{C}=\mathrm{O}$ bonding associated with phosphate dissociation on the surface $[89,96]$.

\subsection{Conclusions}

A novel bottom-up approach for biofabrication of magnetic biochar has been established. During cultivation, fungi can accumulate large amounts of metals and nutrients from municipal wastewaters allowing for a cheap and sustainable method for generation of biomass feedstock. The resulting fungal biomass can be easily processed into magnetic biochar and the physiochemical properties of the materials can be tuned by controlling fungal growth parameters. The magnetic biochars had viable surface areas $\left(51.6-53 \mathrm{~m}^{2} / \mathrm{g}\right)$, average pore sizes $(6.1-13.2 \mathrm{~nm})$, and magnetic capabilities. High phosphorus adsorption capacities of $23.9 \mathrm{mg} / \mathrm{g}$ endorsed the use of these materials in environmental applications. Phosphate adsorption on the chars best fit Freundlich isotherms and the results of FTIR and EDS analyses suggested that substitution of surface oxide groups by phosphate were primarily responsible for adsorption. The fungi derived 
biochars have demonstrated good performance for phosphorus removal and may have numerous applications for adsorption of anionic species such as arsenic from drinking water and chromate for soil remediation. More applications of these magnetic chars are still be explored. 


\section{CHAPTER 4}

\section{ELECTROCHEMICAL CO, REDUCTION TO SYNGAS}

\subsection{Introduction}

Selective and efficient conversion of carbon dioxide $\left(\mathrm{CO}_{2}\right)$ to high energy density fuels is paramount for a closed-carbon energy future. Ideally, renewable energy from solar or wind sources will be used to simultaneously drive $\mathrm{CO}_{2}$ sequestration and transformation into fuels at ambient temperatures without the generation of complex wastes. The injection of an electron into $\mathrm{CO}_{2}$, however, requires highly negative potentials (large energy investment) and the kinetics are quite sluggish under standard conditions [97-99]. As such, synthetic catalysts for the electrocatalytic reduction of $\mathrm{CO}_{2}$ have been studied for several decades [100]. This work has resulted in numerous catalysts (primarily precious and non-precious metals) capable of producing two-electron products such as carbon monoxide $(\mathrm{CO})$, formic acid $(\mathrm{HCOOH})$ and oxalate $\left(\mathrm{C}_{2} \mathrm{O}_{4}{ }^{2-}\right)$ at high yields and efficiencies [101,102]. For example, Peng et al. (2018) recently demonstrated that their preferentially oriented silver nanoparticles could reduce $\mathrm{CO}_{2}$ to $\mathrm{CO}$ with a faradaic efficiency of $96.7 \%$ at a low operating potential of $-0.69 \mathrm{~V}$ vs. RHE [13].

Although promising, products such as $\mathrm{CO}$ are of limited value as they require further processing to be used as fuels or upgraded into other value-added commodities. Consequently, further investigation into the formation of reduction products requiring four or more electrons is necessary. This work inherently poses additional challenges associated with higher over potentials and complex parallel reactions schemes that lead to decreased product specificity [103]. For instance, Hori and co-workers discovered that metallic copper could reduce $\mathrm{CO}_{2}$ to significant amounts of multi-electron products such as methane $\left(\mathrm{CH}_{4}\right)$, ethylene $\left(\mathrm{C}_{2} \mathrm{H}_{4}\right)$ and ethanol $\left(\mathrm{C}_{2} \mathrm{H}_{5} \mathrm{OH}\right)$ [104-106]. Nevertheless, a lack of specificity was evident as more than 14 
different products could be generated by $\mathrm{CO}_{2}$ reduction on copper and improving the precision of product generation has been an prodigious challenge [107].

Recently it was found that cobalt protoporhyrins immobilized on graphite could produce $\mathrm{CH}_{4}$ as one of just three major products [108]. These findings are noteworthy as $\mathrm{CH}_{4}$ can be readily used as a fuel and indicate that an electron transfer involving 8 electrons to $\mathrm{CO}_{2}$ is viable with good precision. Several models based on first principal calculations have since been presented that describe the reaction pathways from $\mathrm{CO}_{2}$ to $\mathrm{CH}_{4}$ on cobalt porphryins $[109,110]$. The culmination of these works suggests that optimizing fundamental parameters such as bulk electrolyte $\mathrm{pH}$, local cation availability and electrolyte cation solvation effects may enhance the overall multi-electron reduction of $\mathrm{CO}_{2}$. For example, an extensive density functional theory (DFT) model developed by Tripkovic et al. proposed that the production of $\mathrm{CH}_{4}$ on cobalt porphryin is a proton-concerted reaction pathway following the formation of an $\left[\mathrm{CoP}-\mathrm{CO}_{2} .\right]^{-}$ adduct (Eq. A.11) [111]. It is then natural to expect that lowering the bulk electrolyte $\mathrm{pH}$ could accelerate the proton-concerted pathway and improve $\mathrm{CH}_{4}$ yields. Though an experimental $\mathrm{pH}$ dependence on $\mathrm{CH}_{4}$ production was noted by others between $\mathrm{pH} 1-3$, it is yet to be expanded to more viable operating conditions (i.e. near neutral conditions)[108]. It is important to note that operating under lower $\mathrm{pH}$ may allow for the hydrogen evolution reaction (HER) to outcompete the $\mathrm{CO}_{2}$ reduction reaction $\left(\mathrm{CO}_{2}-\mathrm{RR}\right)$, decreasing faradaic efficiencies of carbon products [112]. Although an optimal $\mathrm{pH}$ that can balance the affinity of both $\mathrm{HER}$ and $\mathrm{CO}_{2}$ reduction pathways could be determined, increasing the local proton availability (via modified ligand structures) in neutral or alkaline $\mathrm{pH}$ may circumvent these issues. Though this has not been verified using cobalt porphyrins, analogous concepts have been applied to iron porphyrins in aprotic solvents [113]. For instance, a high local concentration of protons via the addition of phenolic hydroxyl 
groups in all phenyl group ortho positions of an iron tetraphenyl porphyrin (FeTPP) monomer greatly enhanced activity and stability for $\mathrm{CO}_{2}$ reduction to $\mathrm{CO}$ and allowed for $\mathrm{CO}$ faradaic efficiencies above $90 \%$ through 50 million turnovers over 4 hours of electrolysis in $\mathrm{N}, \mathrm{N}-$ Dimethylformamide (DMF)[113]. In-line with this discovery, more drastic ligand modifications of FeTPP have also been attempted and have yielded excellent CO faradaic efficiencies (95\%) and cyclability $\left(\mathrm{TOF}=4300 \mathrm{~s}^{-1}\right)[114]$.

The core of these strategies are based on studies that showed the catalytic efficiency and stability of iron porphyrins could be enhanced by the presence of Lewis acids such as $\mathrm{Mg}^{2+}$ or $\mathrm{Ca}^{2+}[115,116]$. These works proposed a push-pull mechanism where the electro-produced reduced species pushes an electron pair to the $\mathrm{CO}_{2}$ molecule and the electron-deficient acid synergist promotes the separation of one of the $\mathrm{C}-\mathrm{O}$ bonds $[117,118]$. Although a comparable mechanism has not been established with cobalt porphyrins, the presence of solvating cations has been proposed to enhance $\mathrm{CO}_{2}$ reduction on cobalt porphryins. For instance, Miyamoto et al. (2018) recently expanded on Koper's quantum chemistry model for water facilitated $\mathrm{CO}_{2}$ reduction mechanisms on a cobalt porphine $(\mathrm{CoP})$ to suggest that additional cation availability in the bulk solution could stabilize the $\left[\mathrm{CoP}-\mathrm{CO}_{2}\right]^{-}$intermediate leading to improved eletroctrocatalytic $\mathrm{CO}_{2}$ reduction efficiencies and lower overpotentials ( Eq. A.23 vs. Eq. A.24) [119].

All things considered, first principles calculations such as these deliver useful insight to direct multi-electron reduction of $\mathrm{CO}_{2}$ on metallic porphyrins but few models have been verified experimentally. The aim of our work was to use information from theoretical models to experimentally improve the electrocatalytic efficiency and yield of multi-electron reduction of $\mathrm{CO}_{2}$ to $\mathrm{CH}_{4}$ on immobilized cobalt porphyrins. An innovative examination of important factors 
such as local proton availability, bulk electrolyte $\mathrm{pH}$, and cation solvation effects was conducted. This included the synthesis and characterization of new catalysts cobalt (II)-5,10,15,20tetrakis(2',6'-dimethoxyphenyl)-21H,23H-porphyrin $\left(\mathrm{Co}\left(\mathrm{o}-\mathrm{CH}_{3}\right) \mathrm{TPP}\right)$ and cobalt(II)-5,10,15,20tetrakis(2',6'-dihydroxyphenyl)-21H,23H-porphyrin (Co(o-OH)TPP). The results presented, hereto, demonstrate high yields and efficiencies for $\mathrm{CO}$ and $\mathrm{CH}_{4}$ under optimized aqueous-based conditions. Furthermore, this work provides valuable experimental insight on existing theoretical models and helps identify key parameters that improve multi-electron reduction under realistic operation conditions.

\subsection{Materials and methods}

\subsection{Catalyst synthesis}

To examine the impact of local proton availability on $\mathrm{CO}_{2}$ reduction, metalloporphyrins $\mathrm{Co}(\mathrm{o}-$ $\left.\mathrm{OCH}_{3}\right)$ TPP and $\mathrm{Co}(\mathrm{O}-\mathrm{OH}) \mathrm{TPP}$ were synthesized according to the reactions schemes in Figure 15 and compared to the performance of Cobalt(II)-5,10,15,20-Tetraphenyl-21H,23Hporphine(CoTPP) purchased from Sigma Aldrich. Porphyrin structures 5,10,15,20-tetrakis(2',6’dimethoxyphenyl)-21H,23H-porphyrin and 5,10,15,20-tetrakis(2',6'-dihydroxyphenyl)21H,23H-porphyrin were produced using comparable techniques to others [120]. Full details of the synthesis reactions are provided in the appendix methods section [A.1]. Briefly, to synthesize $\mathrm{Co}\left(\mathrm{o}-\mathrm{OCH}_{3}\right) \mathrm{TPP}$, solid $\mathrm{H}_{2}\left(\mathrm{o}-\mathrm{OCH}_{3}\right) \mathrm{TPP}(0.1453 \mathrm{~g}, 0.170 \mathrm{mmol})$ and anhydrous $\mathrm{CoCl}_{2}$ (II) $(0.441 \mathrm{~g}, 3.40 \mathrm{mmol})$ were dissolved in anhydrous tetrahydrofuran $(\mathrm{THF}, 30 \mathrm{~mL})$ under inert $\mathrm{N}_{2}$ gas and 2.6-lutidine $(0.060 \mathrm{~mL}, 0.51 \mathrm{mmol})$ was added. The solution was then refluxed for two hours. After removing THF solution, the mixture was dissolved in dichloromethane (DCM) and washed with deionized water three times. Organic solvent was concentrated down and the 
product was collected. $\mathrm{Co}(\mathrm{o}-\mathrm{OH}) \mathrm{TPP}$ was prepared in the same fashion except ethyl acetate was used to dissolve the mixture after removing the THF solution.

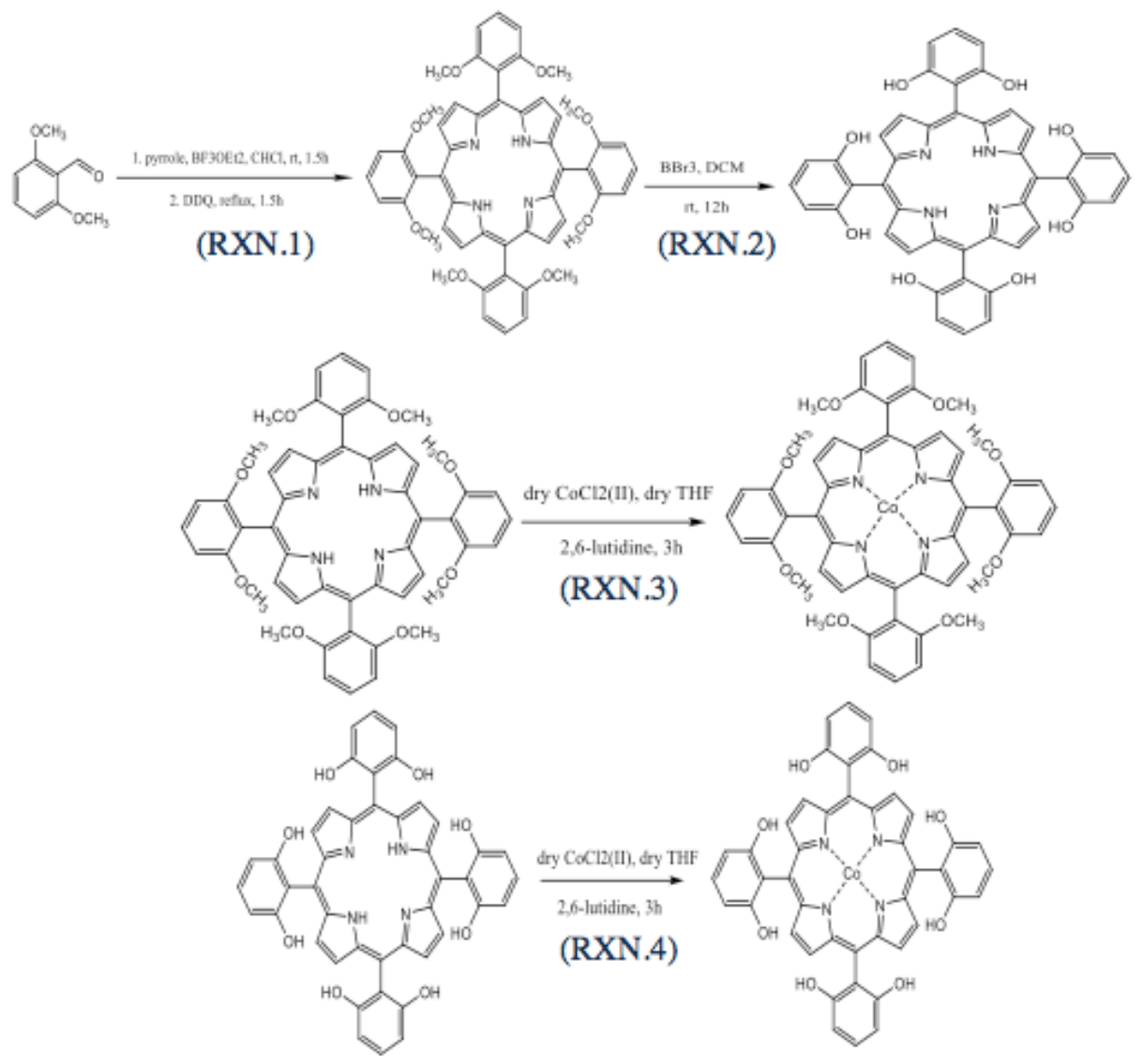

Figure 15: Summary of synthesis reactions for $\mathrm{Co}(\mathrm{o}-\mathrm{OH}) \mathrm{TPP}$ and $\mathrm{Co}\left(\mathrm{o}-\mathrm{OCH}_{3}\right) \mathrm{TPP}$

\subsection{Material characterization}

The as-synthesized porphyrins and metalloporphyrins were characterized by proton nuclear magnetic resonance spectroscopy ( ${ }^{1} \mathrm{H}$ NMR), ultraviolet-visible spectroscopy (UV-Vis), and Fourier-transform infrared spectroscopy (FTIR). ${ }^{1} \mathrm{H}$ NMR spectra were recorded on a Bruker Avance-III 300 NMR spectrometer. Fourier transform infrared (FT-IR) spectra were measured 
with an Agilent Technologies, Cary 630. UV-Vis Absorption spectra were recorded on an Agilent 8453 .

\subsection{Electrochemical testing}

\subsubsection{Preparation of working electrodes}

Glassy carbon (GC) disks (0.3 cm diameter rod, BASi-MF-2012) were used as working electrodes for all homogenous testing conditions. GC disk electrodes were polished using alumina pastes followed by sonication in acetone for 10 minutes prior to use. For heterogeneous testing, a mixture of $5 \mathrm{mg}$ of catalyst and $5 \mathrm{mg}$ of carbon black was sonicated for $30 \mathrm{~min}$ in $2 \mathrm{~mL}$ of DMF. $200 \mu \mathrm{L}$ of the resulting suspension was then drop casted on two sides of carbon paper (Toray-50\% wet-proofed) and dried for 48 hours at room temperature $\left(25 \pm 0.1^{\circ} \mathrm{C}\right)$ in a laminar flow hood.

\subsubsection{Cyclic voltammetry}

Cyclic voltammetry (CV) was carried out using a Pine Research (WaveDriver- 200 series) potentiostat connected in a three-electrode setup. A platinum wire (BASi-MF-1033) was employed as counter electrode along with an $\mathrm{Ag} / \mathrm{AgCl}$ reference electrode and the appropriate working electrode (GC disk or carbon paper). Homogenous testing in DMF was conducted with $2 \mathrm{mM}$ of catalyst and $0.1 \mathrm{M}$ tetrabutylammonium hexafluorophosphate $\left(\mathrm{NBu}_{4} \mathrm{PF}_{6}\right)$ as the supporting electrolyte; heterogeneous testing was conducted using catalyst coated-carbon paper and $0.5 \mathrm{M} \mathrm{NaHCO}_{3}$ as the supporting electrolyte. In some experiments, DMF was mixed with deionized $\mathrm{H}_{2} \mathrm{O}$ in varying concentrations $(2 \mathrm{M}$ and $10 \mathrm{M})$ with $0.1 \mathrm{M} \mathrm{NBu} 4 \mathrm{PF}_{6}$ as the electrolyte. Prior to analysis, the electrolyte solutions were saturated with either pure $\operatorname{Argon}$ or $\mathrm{CO}_{2}$ gas.

\subsubsection{Controlled potential electrolysis}


Controlled potential electrolysis were conducted using the aforementioned potentiostat in combination with a standard Pine Research electrochemical cell (AKCELL1) containing two chambers separated by a glass frit. The cathodic compartment contained catalysts coated-carbon paper as the working electrode (partly submerged in the electrolyte solution) and an $\mathrm{Ag} / \mathrm{AgCl}$ reference electrode with ceramic porous frit (BASi-MW-2030). The anodic compartment had a large graphite electrode $(\mathrm{OD}=0.250 ", \mathrm{~L}=12 ")$. The supporting electrolyte in both chambers contained $0.5 \mathrm{M} \mathrm{NaHCO}_{3}$ dissolved in deionized water. In some experiments, the initial pH was adjusted using $1 \mathrm{M}$ potassium hydroxide or $1 \mathrm{M}$ hydrochloric acid solution and/or $\mathrm{NaCl}$ or $\mathrm{CaCl}_{2}$ were added to the electrolyte. Prior to electrolysis, all electrolytes were saturated with either pure $\mathrm{CO}_{2}$ or argon and the cell was sealed using gas tight rubber seals (Pine Research-14/20; 24/25).

\subsubsection{Gas analysis}

Gas samples were obtained directly from the headspace of the electrochemical cell using a $250 \mu 1$ gastight syringe (Hamilton-1700 series) and were immediately analyzed on an Agilent Technologies 7890A gas chromatography system equipped with a thermal conductivity detector (TCD) and packed column. The oven temperature was a constant $60^{\circ} \mathrm{C}$. Injector and detector temperatures were stable at $150^{\circ} \mathrm{C}$ and $250^{\circ} \mathrm{C}$, respectively. Argon gas was used as the carrier gas at a flow rate of $5 \mathrm{~mL} \mathrm{~min}^{-1}$. $\mathrm{CO}$ and $\mathrm{H}_{2}$ molar concentrations were determined by fitting data to a five-point standard curve prepared for each gas using standard calibration gases.

\subsection{Results and discussion}

\subsection{Material synthesis and characterization}

The as-synthesized porphyrins and metalloporphyrins were characterized by ${ }^{1} \mathrm{H}$ NMR, UV-Vis, and FTIR. The culmination of results indicated successful synthesis of $\mathrm{H}_{2}\left(\mathrm{o}-\mathrm{OCH}_{3}\right)$ TPP and 
$\mathrm{H}_{2}(\mathrm{O}-\mathrm{OH})$ TPP and subsequent metalation reactions to produce $\mathrm{Co}\left(\mathrm{o}-\mathrm{OCH}_{3}\right) \mathrm{TPP}$ and $\mathrm{Co}(\mathrm{o}-$ $\mathrm{OH}$ )TPP, respectively. ${ }^{1} \mathrm{H}$ NMR demonstrated the disappearance of the dimethoxy group at 3.49 ppm indicating the production of $\mathrm{H}_{2}(\mathrm{o}-\mathrm{OH})$ TPP from $\mathrm{H}_{2}\left(\mathrm{o}-\mathrm{OCH}_{3}\right)$ TPP [Fig. A.5, A.6] . This was further supported by FTIR that showed the appearance of a broad peak around $3,300 \mathrm{~cm}^{-1}$, indicating the presence of the hydroxy group [Fig. A.7, A.8]. Successful metalation of the porphyrins was indicated by a shift in the UV-vis Soret absorbance peak from $417 \mathrm{~nm}$ to $413 \mathrm{~nm}$ after metalation [Fig. A.9, A.10]. This was further suggested by FTIR spectra that showed the disappearance of the $\mathrm{N}-\mathrm{H}$ stretching peak around $3,300 \mathrm{~cm}^{-1}$ from both $\mathrm{H}_{2}\left(\mathrm{O}-\mathrm{OCH}_{3}\right) \mathrm{TPP}$ and $\mathrm{H}_{2}(\mathrm{O}-\mathrm{OH}) \mathrm{TPP}$, indicating the substitution of cobalt into the carbon matrixes [Fig. A.7, A.8].

\subsubsection{Impact of local proton source in aprotic solvent}

CVs of $\mathrm{Co}(\mathrm{o}-\mathrm{OCH} 3) \mathrm{TPP}, \mathrm{Co}(\mathrm{o}-\mathrm{OH}) \mathrm{TPP}$, and CoTPP were obtained in a DMF solution containing $0.1 \mathrm{M} \mathrm{NBu}_{4} \mathrm{PF}_{6}$ saturated with $\mathrm{CO}_{2}$ gas to assess the fundamental impact of the local proton source on electrocatalytic $\mathrm{CO}_{2}$ reduction [Fig. 16]. Notably, an assortment of responses in catalytic behavior were observed between the catalysts upon introducing varying quantities of protons $\left(2 \mathrm{M}, 10 \mathrm{M} \mathrm{H}_{2} \mathrm{O}\right)$ to the initially aprotic solvent [Fig. 16]. CoTPP seemed to be unaffected by the addition of water with a $\mathrm{Co}^{2+}-\mathrm{Co}^{+1}$ activation peak around $-0.80 \mathrm{~V}$ vs. $\mathrm{Ag} / \mathrm{AgCl}$ and the start of a major reduction event stable around $-1.85 \mathrm{~V}$ vs. $\mathrm{Ag} / \mathrm{AgCl}$ across each scenario. These findings are similar to those of $\mathrm{Hu}$ et al. (2017) when introducing protons via phenol to CoTPP immobilized on GC disk electrodes in DMF [121]. In contrast, the functionalized catalysts (i.e. $\mathrm{Co}\left(\mathrm{o}-\mathrm{OCH}_{3}\right) \mathrm{TPP}$ and $\left.\mathrm{Co}(\mathrm{o}-\mathrm{OH}) \mathrm{TPP}\right)$ seemed to show enhanced catalytic activity upon the addition of water. For example, the onset potentials for the major reduction event of both $\mathrm{Co}(\mathrm{o}-$ $\mathrm{OH}) \mathrm{TPP}$ and $\mathrm{Co}\left(\mathrm{o}-\mathrm{OCH}_{3}\right)$ TPP seemed to shift from ca. $300 \mathrm{mV}$ to more positive potentials upon adding $10 \mathrm{M} \mathrm{H}_{2} \mathrm{O}$. Remarkably, the reduction current of $\mathrm{Co}\left(\mathrm{o}-\mathrm{OCH}_{3}\right) \mathrm{TPP}$ was much higher than 
others upon the addition of $10 \mathrm{M} \mathrm{H}_{2} \mathrm{O}$ reaching about twice that of CoTPP and Co(o-OH)TPP at $2.5 \mathrm{~V}$ vs. $\mathrm{Ag} / \mathrm{AgCl}$.
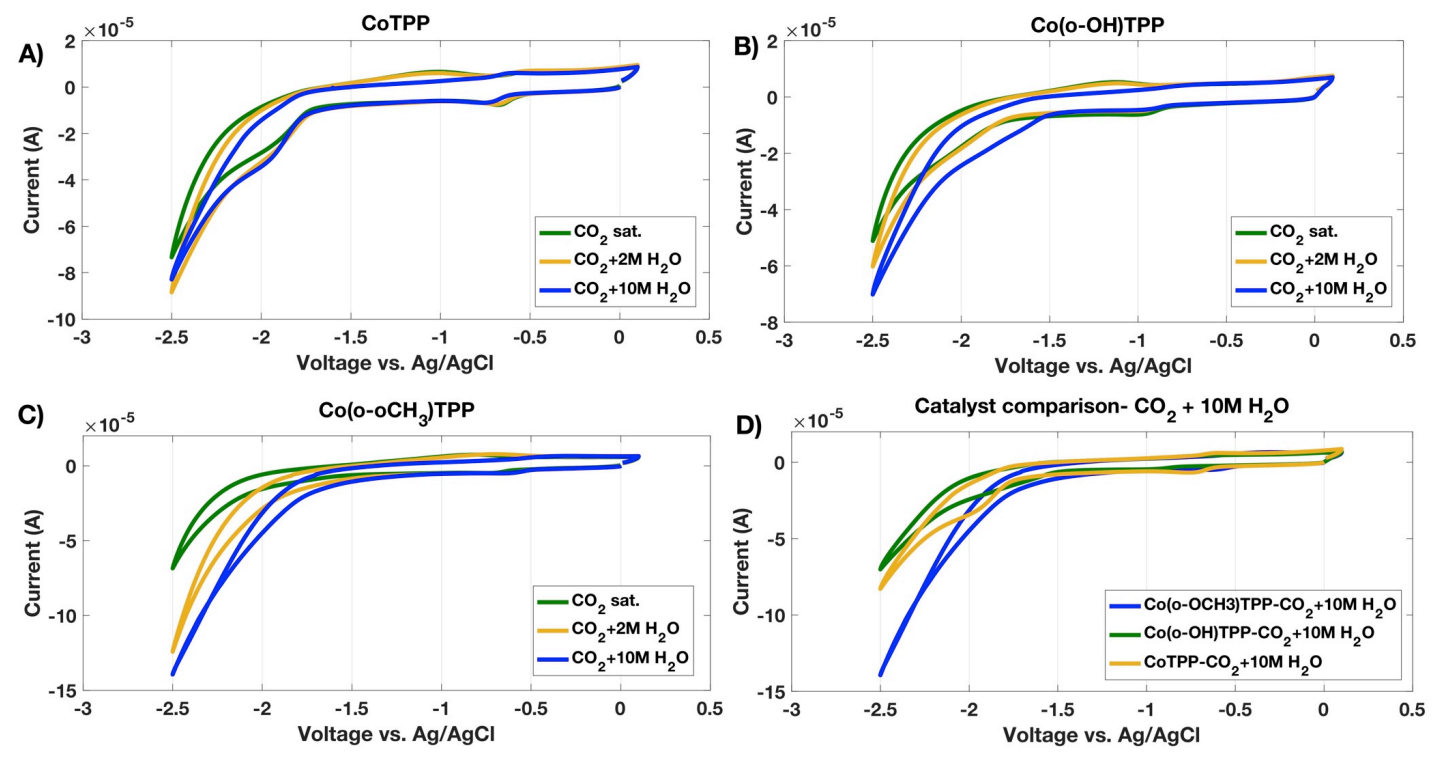

Figure 16: $\mathrm{CO}_{2}$ reduction capabilities of modified cobalt porphyrin structures; $\mathrm{Pt}$ anode, $\mathrm{Ag} / \mathrm{AgCl}$ ref. electrode, glass carbon working electrode; $1 \mathrm{mM}$ catalyst and $0.1 \mathrm{M} \mathrm{NBu} \mathrm{PF}_{6}$ dissolved in DMF; Various amounts of $\mathrm{H}_{2} \mathrm{O}$ added; saturated with $\mathrm{CO}_{2}$

The culmination of these results are in-line with the findings of others using modified FeTPP catalysts for $\mathrm{CO}_{2}$ reduction under homogenous conditions [120]. It is important to note that at the potentials near where the activation peaks start (ca. $-800 \mathrm{mV}$ vs. $\mathrm{Ag} / \mathrm{AgCl}$ ), the current can be attributed to $\mathrm{CO}_{2}$ reduction, HER or a mixture of both. Consequently, controlled potential electrolysis experiments were required to investigate the product selectivity of each catalyst and elucidate the affinity for each possible reaction mechanism.

\subsubsection{Aqueous controlled potential electrolysis}

To maintain the practicality of these findings, electrolysis was conducted under heterogeneous conditions with an aqueous electrolyte (at room temperature) at moderate potentials (-0.85V to - 
$1.5 \mathrm{~V}$ vs. $\mathrm{Ag} / \mathrm{AgCl})$. At each operating voltage it was apparent that the functionalized catalysts (i.e. $\mathrm{Co}\left(\mathrm{o}-\mathrm{OCH}_{3}\right) \mathrm{TPP}$ and $\left.\mathrm{Co}(\mathrm{o}-\mathrm{OH}) \mathrm{TPP}\right)$ favored the HER over the $\mathrm{CO}_{2}-\mathrm{RR}$ in comparison to the bare catalyst, CoTPP. For instance, the faradaic efficiencies (FEs) of CoTPP for HER was only about a tenth of that of $\mathrm{Co}\left(\mathrm{o}-\mathrm{OCH}_{3}\right) \mathrm{TPP}$ or $\mathrm{Co}(\mathrm{o}-\mathrm{OH}) \mathrm{TPP}$ after an hour electrolysis at 1.2V vs. $\mathrm{Ag} / \mathrm{AgCl}[$ Fig. 17].
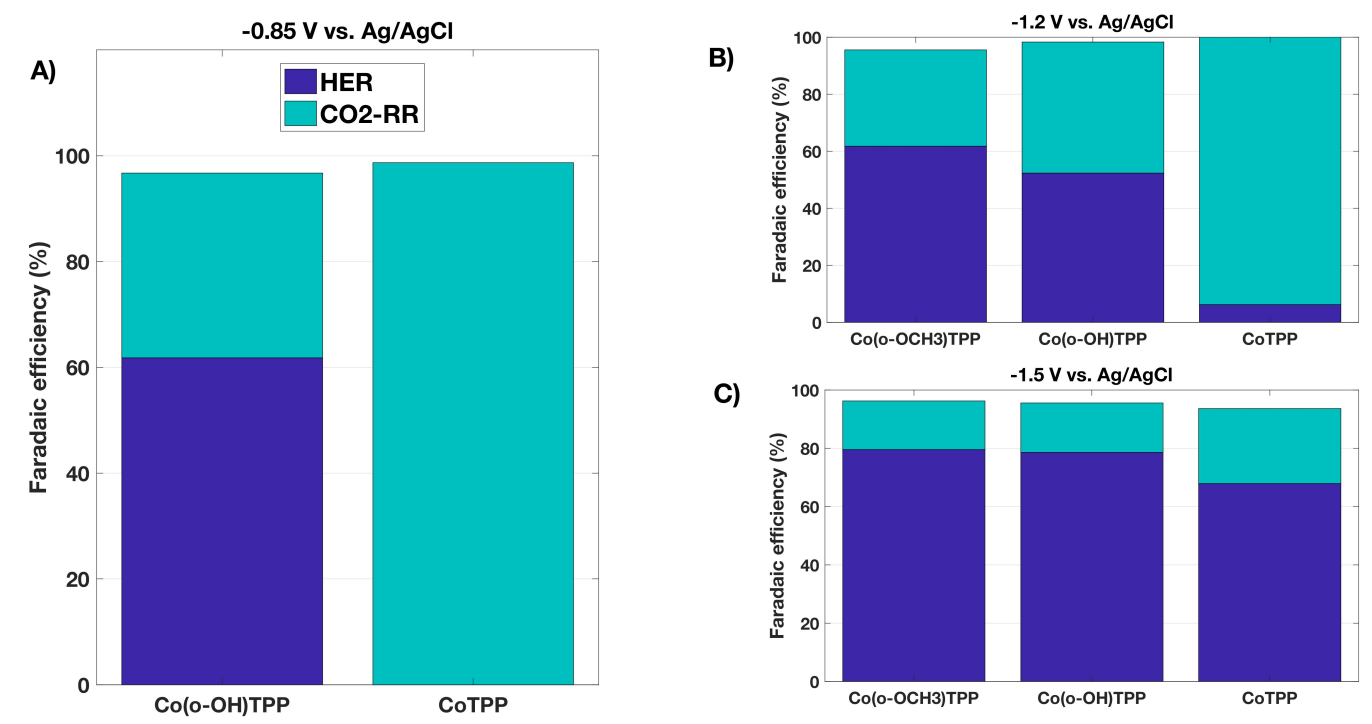

Figure 17: Cobalt porphyrin catalyst preparative scale electrolysis; Faradaic efficiencies of each catalyst at $-0.85 \mathrm{v}$ vs. $\mathrm{Ag} / \mathrm{AgCl}(\mathrm{A})-1.2 \mathrm{v}$ vs. $\mathrm{Ag} / \mathrm{AgCl}(\mathrm{B})-1.5 \mathrm{v}$ vs. $\mathrm{Ag} / \mathrm{AgCl}$ (C) Graphite anode electrode, catalyst deposited on carbon paper working electrode $0.5 \mathrm{M} \mathrm{KHCO}_{3}$ electrolyte; Hydrogen evolution reaction (HER) in dark blue; $\mathrm{CO}_{2}$ reduction reaction $\left(\mathrm{CO}_{2}-\mathrm{RR}\right)$ in light blue

This finding is significant as in comparable studies with iron porphyrins (under homogenous conditions) enhanced catalytic waves were attributed to only $\mathrm{CO}_{2}$ reduction [114]. Using highly regarded DFT models, it was anticipated that the local proton availability would accelerate Eq. A.13 or Eq. A.15 as part of the proton-concerted reaction pathway (Eq. A.9, A.11, A.13, A.15) of $\mathrm{CO}_{2}$ to $\mathrm{CO}$. As the equilibrium potential for Eq. A.15 is high $(-0.37 \mathrm{mV}$ vs. $\mathrm{Ag} / \mathrm{AgCl})$ it is likely that this reaction is spontaneous and non-limiting in the potential range tested, thus the impact of increased proton availability was not observed [109]. The local proton source could 
have been used for Eq. A.13 but it seems that it was outcompeted by initial HER pathways (Eq. A.21 and Eq. A. 22). Interestingly, heterogeneous testing conditions allowed for $\mathrm{CO}_{2}$ reduction to occur at very low over-potentials (ca. $50 \mathrm{mV}$ vs. $\mathrm{Ag} / \mathrm{AgCl}$ ) as noted by others in comparable studies $[121,122]$. At potentials close to the onset (ca. $-0.8 \mathrm{~V}$ vs. $\mathrm{Ag} / \mathrm{AgCl}$ ), $\mathrm{H}_{2}$ pathways were limited and allowed for excellent $\mathrm{CO}_{2}$ reduction efficiencies. For example, CoTPP achieved $98 \%$ $\mathrm{CO}_{2}$-RR FEs compared to $\mathrm{Co}(\mathrm{o}-\mathrm{OH}) \mathrm{TPP}$ which was about $34 \%$ [Fig. 17]. The immobilization of the catalysts on the porous carbon paper may have further exacerbated these efficiencies. Others have also remarked on the importance of strong electrode-catalyst interactions for multielectron $\mathrm{CO}_{2}$ reduction $[123,124]$. Interestingly, the $\mathrm{CO}_{2}$ reduction capabilities of the bare catalysts (CoTPP) were not improved by addition of local proton sources. This suggests that improving proton-concerted pathways may not be effective in enhancing $\mathrm{CO}_{2}$ reduction on cobalt porphyrins. As far as $\mathrm{CH}_{4}$ production, several reduction pathways are currently under review and the $\mathrm{pH}$ dependence is not yet clear in neutral or alkaline conditions $[110,125,126]$.

\subsection{Bulk electrolyte pH}

The role of the bulk electrolyte $\mathrm{pH}$ was examined in the range of 6.5-9.5 to examine the impact on $\mathrm{CO}_{2}$ reduction on CoTPP in heterogeneous alkaline and neutral $\mathrm{pH}$ conditions [Fig. 18]. It seemed the $\mathrm{pH}$ of the solution did not greatly impact the $\mathrm{CO}_{2}$ reduction efficiencies of CoTPP as the $\mathrm{CO}_{2}-\mathrm{RR}$ efficiencies were all between 95\%-99\% throughout the range tested (6.5-8.5). This suggests that $\mathrm{H}_{2} \mathrm{O}$ is a suitable reactant in reduction pathways, such as Eq. A.24, in place of $\mathrm{H}^{+}$as suggested by the first principle calculations of others $[108,127]$. Notably, the onset potential at pH 9.5 shifted to lower potentials and only about half the current was drawn at $-0.85 \mathrm{~V}$ vs. $\mathrm{Ag} / \mathrm{AgCl}$ compared to the other samples [Fig. 18]. This may suggest that the presence of anions can actually interrupt $\mathrm{CO}_{2}$ reduction pathways and extreme alkaline operating conditions should 
be avoided. This is likely because at higher $\mathrm{pH}$ the speciation of a key intermediate $\mathrm{CoP}-\mathrm{COOH}^{-}$ $(\mathrm{pKa}=8.3)$ changes and favors sequential proton-electron transfer rather than concerted [110]. The sequential proton-electron transfer reaction steps are likely more sluggish and lead to decreased observed current uptake and gas production.
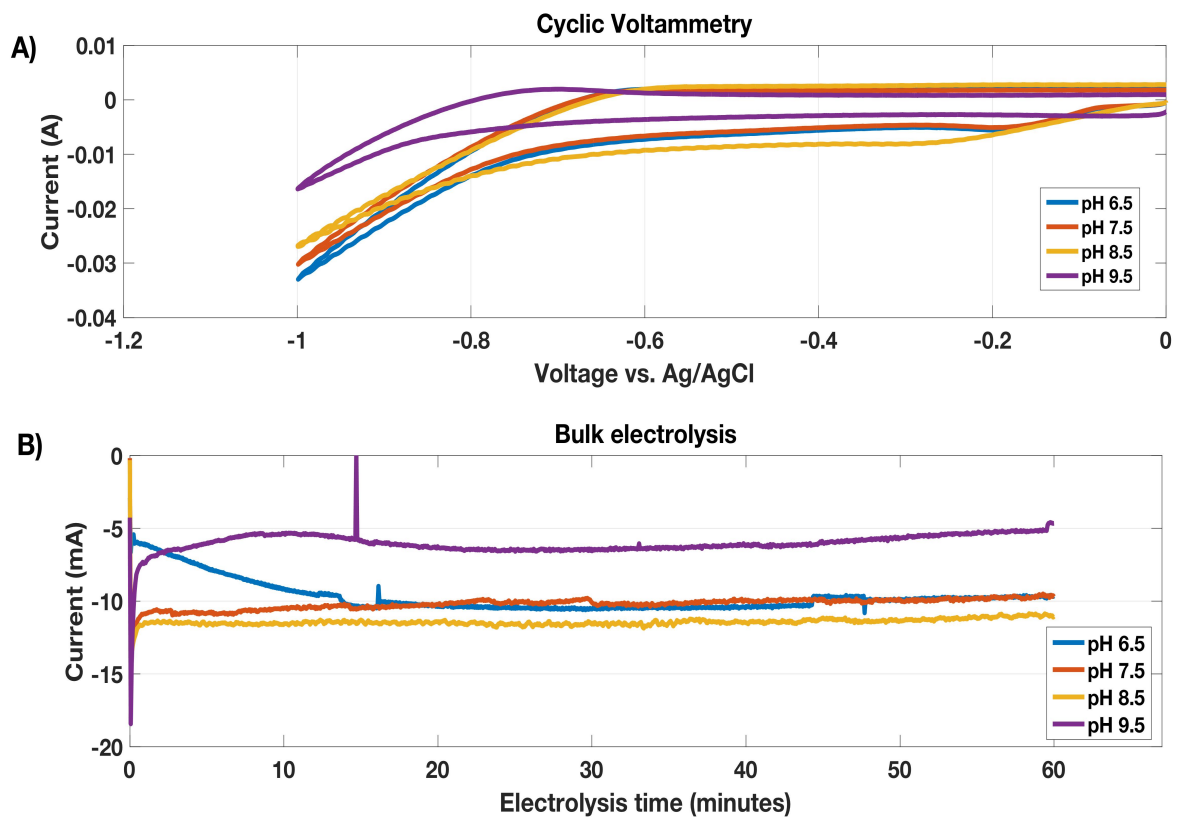

Figure 18: Bulk electrolyte $\mathrm{pH}$ effect on CoTPP $\mathrm{CO}_{2}$ reduction; Cyclic voltammetry at various $\mathrm{pH}$ (A) Current vs. time plot; potential at $-0.85 \mathrm{~V}$ vs. $\mathrm{Ag} / \mathrm{AgCl}$ (B)

\subsection{Influence of alkali cations on $\mathrm{CO}_{2}$ reduction}

\subsubsection{Electrolyte cation effects}

As the availability of protons showed to have little improvement on $\mathrm{CO}_{2}$ reduction, an investigation of the presence of other alkali cations was conducted to improve $\mathrm{CO}_{2}$ reduction on CoTPP. Controlled potential electrolysis of CoTPP on carbon paper in $0.5 \mathrm{M} \mathrm{NaHCO}$ were compared to $0.5 \mathrm{M} \mathrm{KHCO}_{3}$. In theory, both potassium $\left(\mathrm{K}^{+}\right)$and sodium $\left(\mathrm{Na}^{+}\right)$would be absorbed to the negatively charged electrode through electrostatic attraction. $\mathrm{K}^{+}$, however, is a larger 
molecule than $\mathrm{Na}^{+}$(atomic radii: $138 \mathrm{pm}$ vs. $102 \mathrm{pm}$ ) and thus has a smaller hydration number $(7$ vs. 13) [128-130]. As a result, $\mathrm{Na}^{+}$has a larger geometric configuration that would potentially make it more difficult for $\mathrm{H}^{+}$to interact with the electrode, leading to decreased $\mathrm{H}_{2}$ production. Nevertheless, this seemed to have little effect as samples containing both $0.5 \mathrm{M} \mathrm{NaHCO}$ and $\mathrm{KHCO}_{3}$ achieved similar $\mathrm{CO}$ and $\mathrm{CH}_{4}$ production [Fig. 19]. To further examine solvation effects, addition of monovalent and divalent cations were also compared by addition of $0.5 \mathrm{M} \mathrm{Na}^{+}$and $0.5 \mathrm{M} \mathrm{Ca}^{+2}$ to an electrolyte solution containing $0.5 \mathrm{M} \mathrm{NaHCO}_{3}$. Interestingly, the addition of $\mathrm{Ca}^{+2}$ lead to very poor $\mathrm{CO}_{2}$ reduction efficiencies and slow deactivation of the catalyst was observed likely via precipitation of $\mathrm{CaCO}_{3}$ on the electrode surface. In contrast, the addition of $\mathrm{Na}^{+}$significantly improved the yield of both $\mathrm{CO}$ and $\mathrm{CH}_{4}$.
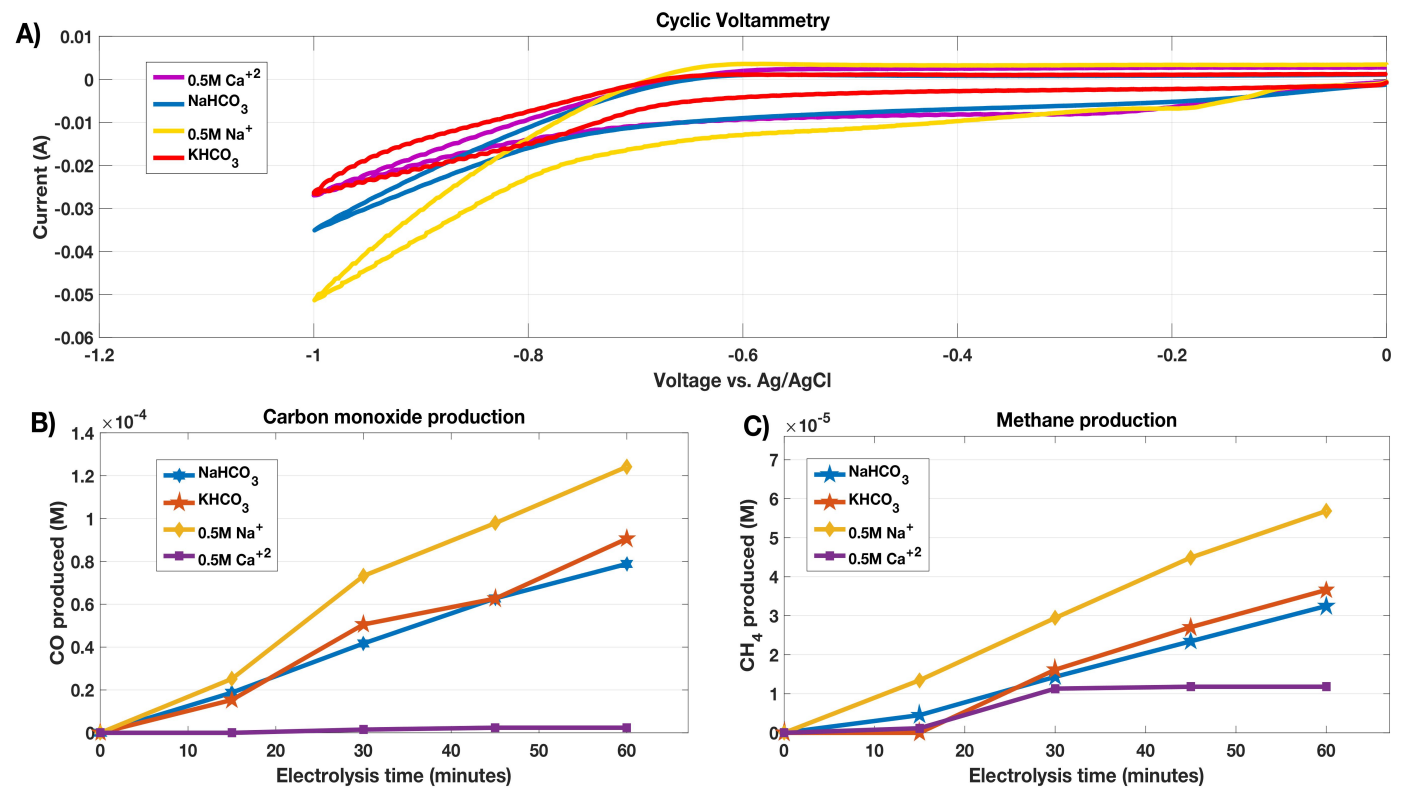

Figure 19: Solvating cation effect on CoTPP $\mathrm{CO}_{2}$ reduction; $0.5 \mathrm{M} \mathrm{NaHCO}$ alone or with $0.5 \mathrm{M}$ Nacl or $0.5 \mathrm{M} \mathrm{Ca}^{+2}$ added; $0.5 \mathrm{M} \mathrm{KHCO}_{3}$ electrolyte; Cyclic voltammetry $0.1 \mathrm{v} / \mathrm{s}$ (A) Carbon monoxide production (B) Methane production (C) Graphite anode electrode, catalyst deposited on carbon paper working electrode

For example, almost 1.6 times more mmoles of $\mathrm{CO}$ were produced in the electrolyte containing 
$0.5 \mathrm{M} \mathrm{NaHCO}_{3}$ upon the addition of $0.5 \mathrm{M} \mathrm{Na}^{+}$. This was likely due to a combination of steric and electronic effects at the electrode surface. First principle calculation models developed by Miyamoto et al. (2018) suggest that $\mathrm{Na}^{+}$can act as a Lewis acid stabilizing the key intermediate $\left[\mathrm{CoP}-\mathrm{CO}_{2}\right]^{-}$in $\mathrm{CO}_{2}$ reduction to $\mathrm{CO}$ [119] This manifests itself as a higher equilibrium reaction potential (-0.74 vs. $-1.33 \mathrm{~V}$ vs. RHE) for the solvated $\mathrm{H}_{2} \mathrm{O}$ (Eq. A.23) vs. the bare molecule (Eq.A.24 ) when participating in reaction Eq. A.13 or A.14. Furthermore, the hydration of $\mathrm{Na}^{+}$ may have also inhibited $\mathrm{H}^{+}$diffusion to electrode surface by the steric hindrances.

To further evaluate these effects, the impact of the sodium was further examined by testing concentrations in the range of $0.0-1.0 \mathrm{M} \mathrm{Na}^{+}$added to $0.5 \mathrm{M} \mathrm{NaHCO}_{3}$ solutions [Fig. 20]. As expected, increased $\mathrm{Na}^{+}$concentration lead to increased product yield. Interestingly, $\mathrm{CO}$ production seemed to drop in favor of methane production. This may indicate that the $\mathrm{Na}^{+}$ stabilization effects are more important for the $\mathrm{CO}$ adduct stabilization than following reactions. Ultimately, the highest yield of .0082 mmoles $\mathrm{CH}_{4}$ were achieved after addition of $1.0 \mathrm{M} \mathrm{Na}$. This presents excellent performance compared to copper(II)-5,10,15,20-tetrakis(2,6dihydroxyphenyl)porphyrin at $-1.1 \mathrm{~V}$ vs. $\mathrm{Ag} / \mathrm{AgCl}$ under heterogeneous conditions [131]. 

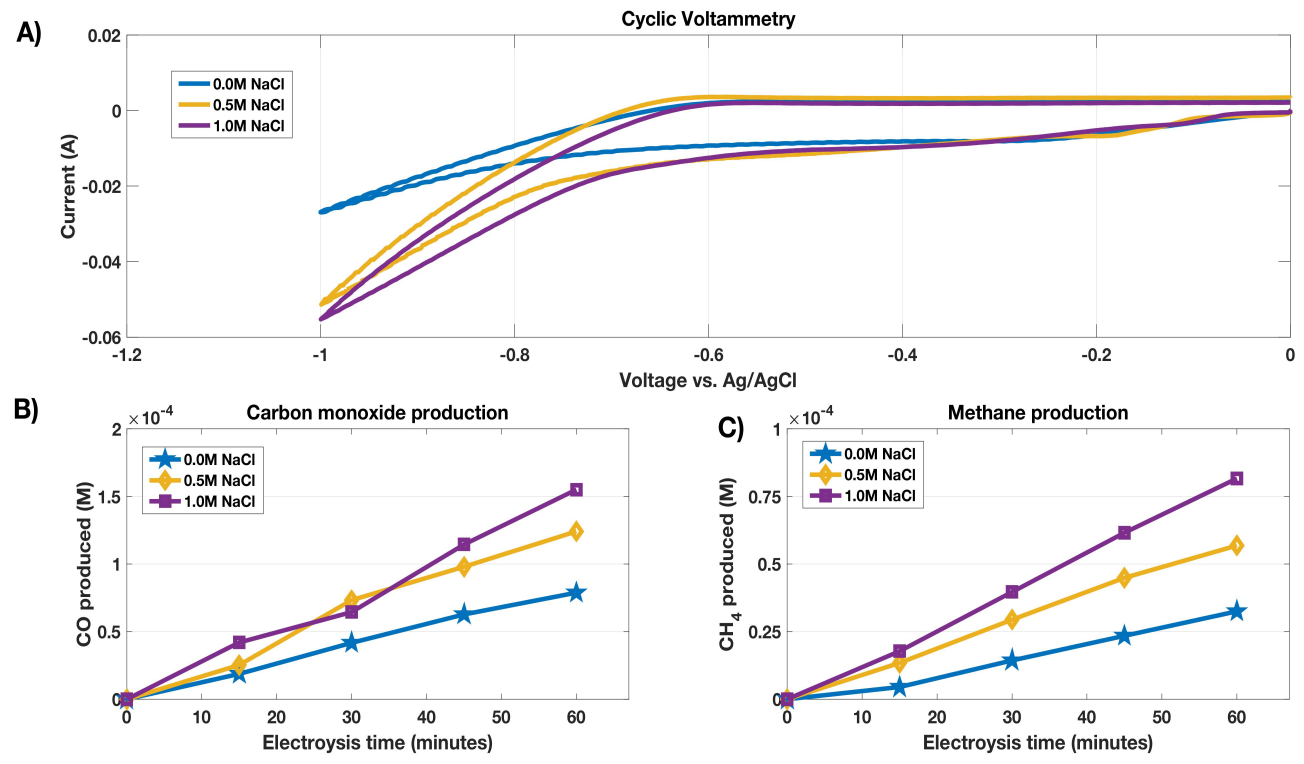

Figure 20: Sodium stabilization effect on $\mathrm{CoTPP} \mathrm{CO}_{2}$ reduction; $0.5 \mathrm{M} \mathrm{NaHCO}$ alone or with $0.5 \mathrm{M}$ or $1.0 \mathrm{M} \mathrm{Na}^{+1}$ added; Cyclic voltammetry $0.1 \mathrm{v} / \mathrm{s}$ (A) Carbon monoxide production (B) Methane production (C) Graphite anode electrode, catalyst deposited on carbon paper working electrode

\subsection{Conclusions}

Cobalt porphyrins immobilized on carbon paper can produce $\mathrm{CO}$ and $\mathrm{CH}_{4}$ in an aqueous electrolyte at near neutral $\mathrm{pH}$ and at a low over-potential of ca. $50 \mathrm{mV}$. For the first time, the impact of increased local proton availability via modified CoTPP was examined under heterogeneous conditions. This lead to accelerated $\mathrm{H}_{2}$ production and suggested $\mathrm{CO}$ and $\mathrm{CH}_{4}$ production pathways are not $\mathrm{pH}$ sensitive in near neutral $\mathrm{pH}$ conditions. This was supported by bulk electrolyte $\mathrm{pH}$ optimization that showed little effect on $\mathrm{CO}_{2}$ reduction activity. Sodium solvation increased $\mathrm{CO}_{2}$ reduction and high yields and almost $98 \%$ carbon products were achieved with addition of $1.0 \mathrm{M} \mathrm{Na}^{+}$. The culmination of these findings has helped identified key operational parameters to facilitate multi-electron transfer to $\mathrm{CO}_{2}$ under realistic and scalable conditions. 


\section{CHAPTER 5}

\section{TWO STAGE BIOHYBRID SYSTEM FOR ELECTROCHEMCIAL SYNGAS PRODUCTION AND MICRBOBIAL CONVERSION}

\subsection{Introduction}

Carbon dioxide $\left(\mathrm{CO}_{2}\right)$ is a major contributor to atmospheric "green-house" gases that are fundamentally accountable for global climate change. Currently, the atmospheric $\mathrm{CO}_{2}$ concentration is about 410 ppm (3,200 gigatons) and continues to rise by ca. 2 ppm per year [132]. Carbon is the primary component of many everyday fuels, chemicals, and materials and sectors such as energy, agricultural, and transport account for more than 27 billion tons of $\mathrm{CO}_{2}$ emissions each year [133]. As such, a platform that that utilizes $\mathrm{CO}_{2}$ as the primary feedstock for the synthesis of carbon commodities would be ideal for a closed carbon economy. In addition to generating important environmental and societal impacts, such techniques will also yield incredible economic benefits by taking advantage of impending governmental carbon tax incentives as well as the large margin of upgrading a cheap and abundant carbon source to high value commodities [134].

In the past, approaches for converting $\mathrm{CO}_{2}$ to carbon products has involved the use of photosynthetic carbon assimilation via plants, algae or cyanobacteria $[135,136]$. The scale-up of such techniques is, however, inherently limited by low energy to biomass conversion efficiencies as well as large water and land use requirements [137]. Alternatively, tremendous efforts have been made to develop non-biological catalytic processes for the conversion of $\mathrm{CO}_{2}$ into products, however, most of these devices have demonstrated substantial drawbacks such as low product selectivity, high temperature requirements, or generation of toxic wastes $[138,139]$. In recent

years, some have shifted their focus to the integration of non-biological and biological 
conversion techniques that aim to pair the advantages of both processes [140]. This work has coincided with considerable advances in solar and wind power that can now offer sources of low-cost and sustainable electricity to drive such devices.

One area that has received a lot of attention in the past decade is microbial electrosynthesis (MES) in which electrons are transferred to microbes via extra-cellular electron transfer (EET). Though several MES designs have demonstrated high-energy efficiencies (up to $90 \%$ ), this technology has been fundamentally challenged by EET mechanisms that can only accommodate low current densities (at most $10 \mathrm{~mA} / \mathrm{cm}^{2}$ ) which result in low production rates (i.e. $<$ 1g/l/day) [19,141]. To by-pass these limitations, other biohybrid approaches have been developed that involve the electrochemical generation of an electron donor that is fed to the microbe rather than using the electrode itself. For example, in a single-pot system $\mathrm{H}_{2}$ produced by a NiMoZn alloy catalyst was fed to Ralstonia eutropha and aerobically converted to isopropanol at high yields $(216 \mathrm{mg} / \mathrm{L})$ [142]. Though promising, high overpotentials were needed to maintain microbial growth as toxic reactive oxygen species were generated at potentials near optimal $\mathrm{H}_{2}$ production [142]. Furthermore, the energy efficiencies of approaches that rely on aerobic bacteria, like Ralstonia eutropha, can only achieve limited energy efficiencies as a large portion of the feedstock electrons must end up reducing oxygen $\left(\mathrm{O}_{2}\right)$ to water rather than in the product. Alternatively, anaerobic bacteria such as acetogens can offer very high conversion of substrates to product (70-90\%) as they utilize a reductive acetyl-CoA pathway that provides energy to the cell while assimilating and reducing $\mathrm{CO}_{2}$ or $\mathrm{C} 1$ carbons [143]. An example of such a microbe is Clostridium ljungdahlii, which has the unique capability to convert $\mathrm{CO}$ or $\mathrm{CO} / \mathrm{CO}_{2}$ into high value products such as ethanol with excellent conversion efficiencies via the Woodljungdhali pathway [144]. This is advantageous because $\mathrm{CO}$ is a preferred substrate to $\mathrm{H}_{2}$ as it 
can partition into liquid phase easier for microbial uptake and releases more Gibbs free energy when used, leading to generation of more reduced products [144]. From an electrochemical standpoint, $\mathrm{CO}$ is also a preferred intermediate to $\mathrm{H}_{2}$ as it directly utilizes $\mathrm{CO}_{2}$ and can be produced at high efficiencies and selectivity. For example, Pan et al. (2018) recently demonstrated that their atomically dispersed Co-N5 site anchored on polymer-derived hollow Ndoped porous carbon spheres could produce $\mathrm{CO}$ at faradaic efficiencies of almost $99 \%$ at low overpotentials (-0.73 vs. RHE) [145].

In this work, we propose a two-stage biohybrid system for $\mathrm{CO}_{2}$ conversion into carbon commodities. In the first stage, $\mathrm{CO}_{2}$ is fed to a gas diffusion $\mathrm{CO}_{2}$ electrolysis cell that electrochemically converts $\mathrm{CO}_{2}$ to $\mathrm{CO}$. The effluent gas mixture is then fed to a downstream bioreactor containing Clostridium ljungdahlii that converts the feed gas into value-added commodities. The results presented, hereto, describe the development and optimization of this novel biohybrid system.

\subsection{Materials and methods}

\subsection{1. $\mathrm{CO}_{2}$ electrolyzer construction and operation}

The $\mathrm{CO}_{2}$ electrolyzer consisted of a membrane electrode assembly $\left(5 \mathrm{~cm}^{2}\right)$ mounted in fuel cell hardware purchased from Dioxide Materials (Boca Raton, FL). Both the anode and cathode electrodes were prepared by depositing nanoparticle inks onto carbon diffusion layers (Sigracet 35 BC GDL, Ion Power). The cathode ink was synthesized by adding silver nanoparticles (30 $\mathrm{mg})$ to distilled water $(0.1 \mathrm{~mL})$ and isopropanol $(0.2 \mathrm{~mL})$. The mixture was then sonicated for 1 min and spray-painted onto a $2.5 \mathrm{~cm} \times 2.5 \mathrm{~cm}$ square cut out of carbon gas diffusion layer. The anode electrode was prepared in an similar fashion except it was coated with iridium oxide (30mg) rather than silver and 5\% PTFE solution was added to the ink as a binder. A Sustainion 
imidazolium-functionalized polymer membrane was sandwiched between the as prepared electrodes and the entire membrane-electrode assembly was mounted between two gaskets. The anode (titanium) and cathode (stainless steel) flow fields were then attached on the respective sides using bolts and ring terminals were screwed into both outer plates. For all tests, an anolyte solution ( $\mathrm{pH}=7$ ) consisting of $10 \mathrm{mM} \mathrm{KHCO}_{3}$ was circulated at $3 \mathrm{~mL} / \mathrm{min}$ with a peristaltic pump through the anode chamber. On the cathode side, a gas cylinder attached to a mass flow controller delivered $\mathrm{CO}_{2}\left(99 \%\right.$ purity) at $35 \mathrm{~mL} / \mathrm{min}$ to a $\mathrm{CO}_{2}$ humidifier bottle containing $100 \mathrm{~mL}$ of deionized water. The effluent of the humidifier was connected directly to the inlet of cathode chamber. The electrochemical cell was connected to a DC-power supply [Circuit Specialists-3600series] that was operated under constant current conditions while the voltage was carefully monitored [Fig. 21].

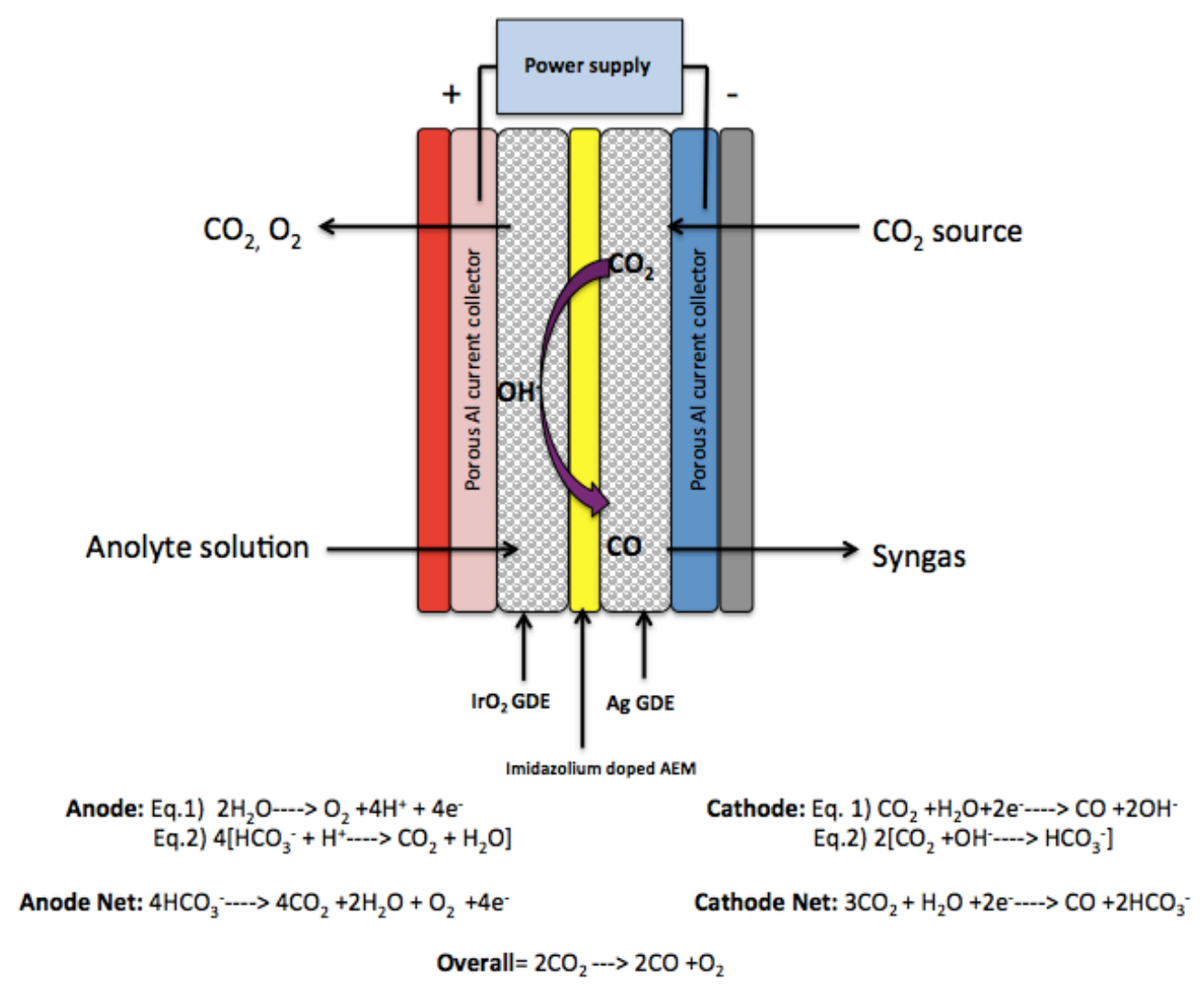

Figure 21: Detailed schematic of $\mathrm{CO}_{2}$ electrolyzer and associated reactions 


\subsubsection{Microbial culturing experiments}

All microbial cultures were maintained in $162.5 \mathrm{~mL}$ Wheaton serum bottles (Borosilicate glass, Fisher Scientific, UK) containing $30 \mathrm{~mL}$ of rich YTF media $[144,146]$. The initial $\mathrm{pH}$ of the media was then adjusted to 5.8 using a concentrated $1 \mathrm{M}$ solution of potassium hydroxide [144]. Prior to autoclaving $\left(121^{\circ} \mathrm{C}\right.$ for 30 minutes), ultra high purity argon gas (General Air Service Supply- UN1006) was diffused into the liquid media for approximately 45 minutes to remove any oxygen. Gas impermeable butyl rubber septum-type stoppers and aluminum crimp seals were then used to seal the bottles and the headspaces were purged with argon for another 10 minutes. After autoclaving, the bottles were cooled to room temperature $\left(25^{\circ} \mathrm{C}\right)$. Bottles were then purged with a filtered (PTFE $0.2 \mu \mathrm{m})$ gas mixture of $\mathrm{H}_{2} / \mathrm{CO}_{2}(25 / 75 \%)$ for approximately 35 minutes. In some cultures, fructose $(5 \mathrm{~g} / \mathrm{L})$ was also added from a stock solution. Prior to inoculation, cultures were then reduced by addition of $0.5 \mathrm{~mL}$ of the reducing agent to make final concentrations of $3.1 \mathrm{mM}$ L-cysteine-HCl. Inocula $(\mathrm{OD}=0.5)$ from a previous culture were then added to the bottles in a 1 to 10 ratio. All cultures were incubated horizontally on an orbital shaker incubator, set at $200 \mathrm{rpm}$ and $37^{\circ} \mathrm{C}$.

\subsubsection{Clostridium ljungdahlii genetic engineering}

A CRISPR-Cas9 tool tailored for C. ljungdahli, was used to generate a mutant lacking phosphotransacetylase (pta). Pta converts acetyl-CoA to acetyl-phosphate, the deletion of which will increase the pool of acetyl-CoA toward products such as ethanol or pyruvate derived metabolites. The CRISPR-Cas9 plasmid specifically targeted the pta gene with its deletion verified using PCR. Further details of the methods are provided elsewhere [147]. 


\subsubsection{Two-stage biohybrid system operation}

Figure 22 describes the full setup of the biohybrid system. The effluent gas from the $\mathrm{CO}_{2}$ electrolyzer was passed through an empty condensation bottle $(100 \mathrm{~mL})$ with a gas tight lid to dry the gas. The gas was then passed through a filter ( $2 \mu \mathrm{m}$ PTFE) and directed through the lid of a bioreactor bottle $(100 \mathrm{~mL})$ that was submerged in a water bath controlled with a heating jacket and module set to 37 (deg. C) [51]. The water bath and bioreactor were placed on a magnetic stir plate that agitated the media in the reactor bottle at $375 \mathrm{rpm}$. The headspace of the bioreactor was pumped via peristaltic pump into a condensation collector at $15 \mathrm{~mL} / \mathrm{min}$ and the final effluent was released to atmosphere in a standard fume hood.

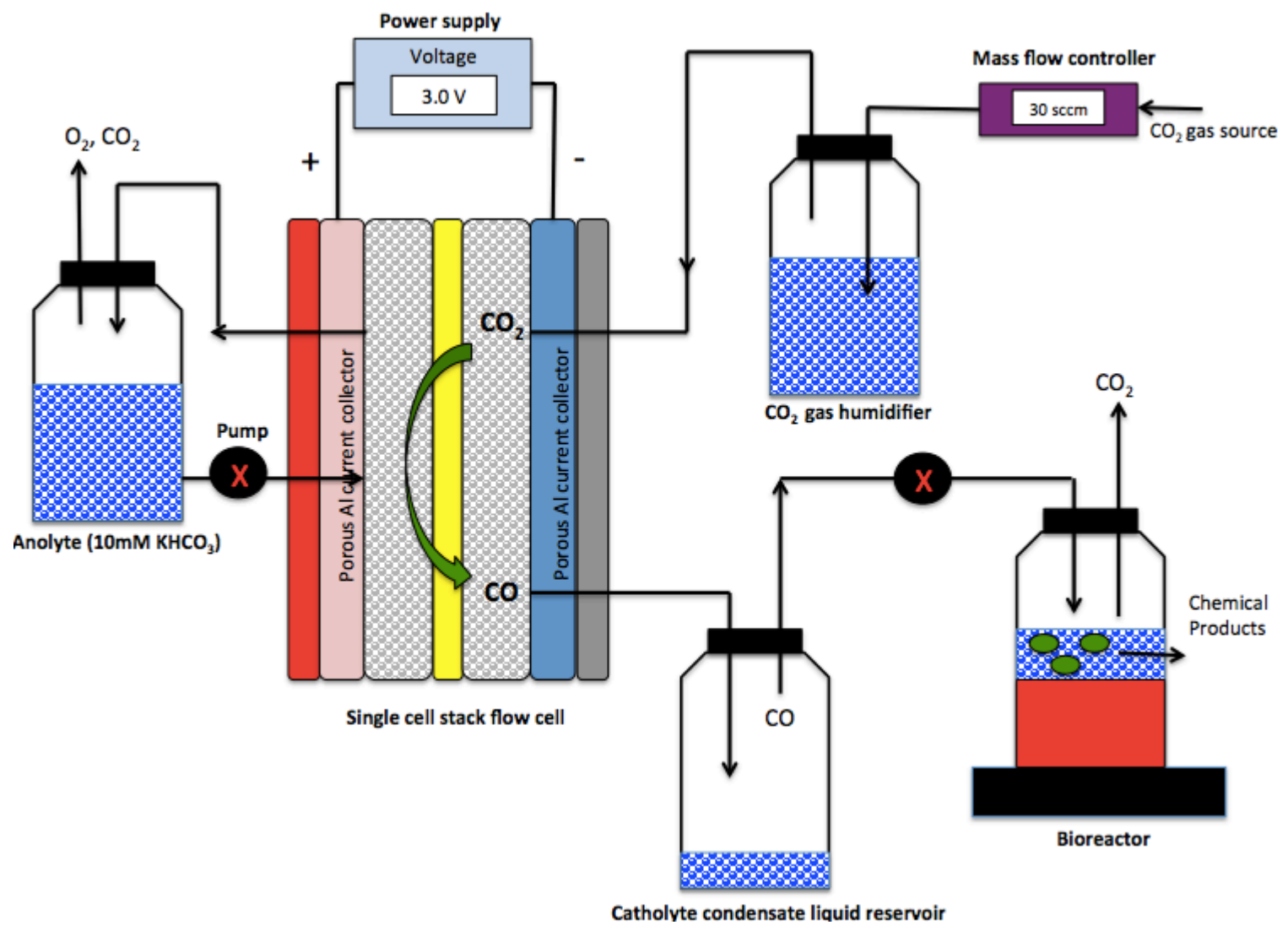

Figure 22: Schematic of two-stage biohybrid system operating process 


\subsubsection{Gas and liquid analyses}

For electrochemical performance analyses, the $\mathrm{CO}_{2}$ electrolyzer was operated for at least 2.5 hours to reach steady state before sampling. After this time tedlar bags (Supel-Inert Foil) were attached directly to the effluent of the cathode side and filled for 33 minutes to fill the bag. Gas

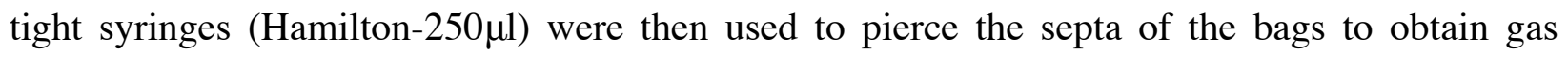
samples. $\mathrm{H}_{2}$ and $\mathrm{CO}$ molar headspace concentrations were determined by manually injecting $250 \mu$ l of sample into a gas chromatograph (Agilent Technologies-7890A) equipped with a thermal conductivity detector (TCD) and packed column [144]. The oven temperature was a constant $60^{\circ} \mathrm{C}$. Injector and detector temperatures were stable at $150^{\circ} \mathrm{C}$ and $250^{\circ} \mathrm{C}$, respectively. Helium gas was used as the carrier gas at a flow rate of $5 \mathrm{~mL}$ min- $1 . \mathrm{CO}_{2}$ molar headspace concentrations were monitored by manually injecting $100 \mu \mathrm{l}$ of sample into a gas chromatograph (Hewlett Packard-5890) equipped with a thermal conductivity detector (TCD) and packed column [144]. The oven temperature was a constant $60^{\circ} \mathrm{C}$. Injector and detector temperatures were stable at $150^{\circ} \mathrm{C}$ and $190^{\circ} \mathrm{C}$, respectively. Helium gas was used as the carrier gas at a flow rate of $30 \mathrm{~mL}$ min-1. Molar concentrations were determined by fitting data to a five-point standard curve prepared for each gas.

During fermentation experiments, liquid samples $(1 \mathrm{~mL})$ were withdrawn using a sample tube submerged in the media. The liquid samples were analyzed for optical density (600nm) as well as acetate and ethanol content. Optical density measurements were made using a spectrophotometer (Beckman Coulter-DU800) at a fixed wavelength of $600 \mathrm{~nm}$ and converted to dry cell densities using a standard calibration curve. Metabolites were analyzed via high-pressure liquid chromatography [144]. Prior to analysis, liquid samples were centrifuged at 14000rpm for 10 minutes and the supernatant was passed through $0.22 \mu \mathrm{m}$ filters. Organic acids were separated 
on an Aminex HPX-87H X column with $4 \mathrm{mM} \mathrm{H}_{2} \mathrm{SO}_{4}$ as the eluent. Molar concentrations were determined by fitting peak areas to a standard curve prepared by diluting ethanol fermentation analytical standard solutions (Bio-Analaytical-LQC-NREL-50).

\subsection{Results and discussion}

\subsubsection{Electrochemical performance evaluation}

Galvanostatic $\mathrm{CO}_{2}$ electrolysis tests were conducted under various current densities to evaluate the performance of the gas diffusion electrolysis module. At $80 \mathrm{~mA} / \mathrm{cm}^{2}$, only a small amount of $\mathrm{CO}$ was produced while the faradaic efficiency for hydrogen reached about $60 \%$. This amounted to a hydrogen generation rate of $4.2 \mathrm{mmol} / \mathrm{hr}$ [Fig. 23]. As current density was increased, the faradaic efficiencies for $\mathrm{CO}$ as well as the $\mathrm{CO}$ production rates increased dramatically. For instance, at a current density of $200 \mathrm{~mA} / \mathrm{cm}^{2}$ almost no hydrogen was evolved and the faradaic efficiency for $\mathrm{CO}$ was over $80 \%$. This translated to a $\mathrm{CO}$ production rate over $15.0 \mathrm{mmol} / \mathrm{hr}$.

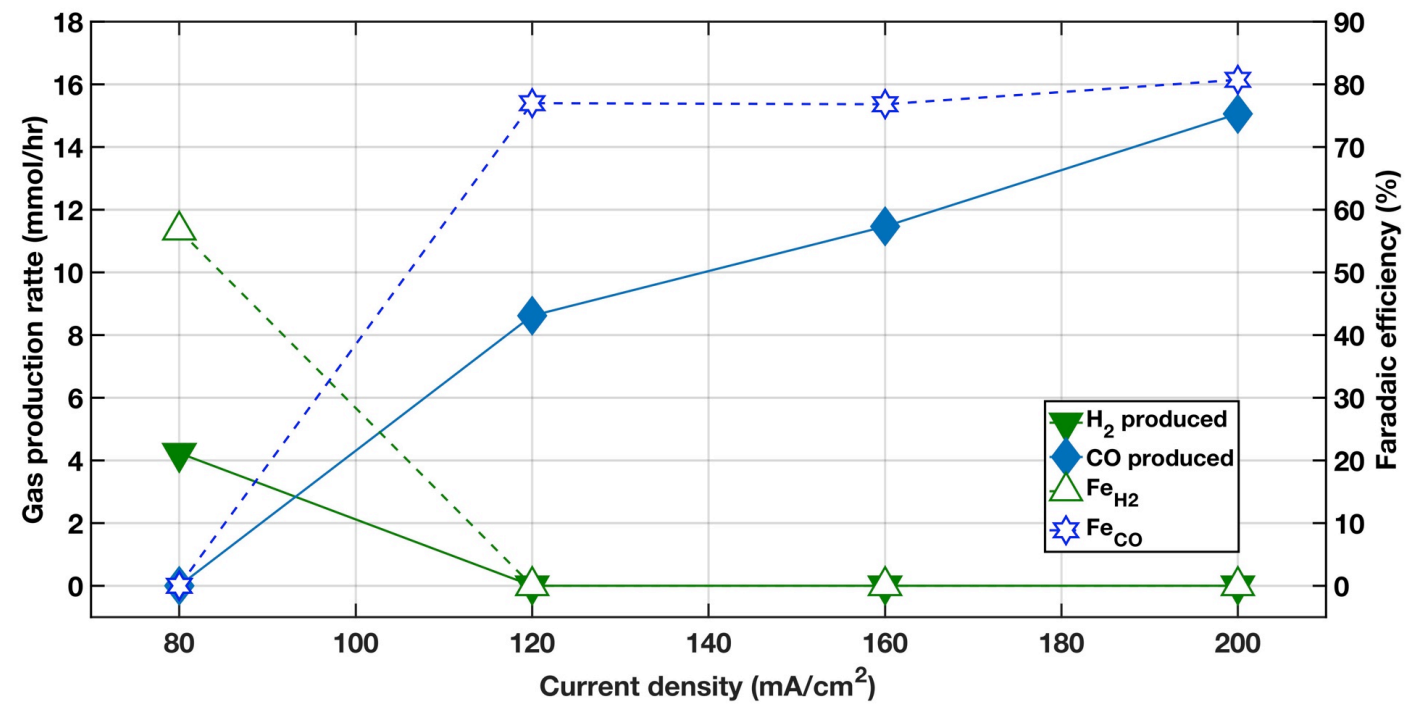

Figure 23: Gas production and faradaic efficiency at variable current densities; $10 \mathrm{mM} \mathrm{KHCO}_{3}$ electrolyte anolyte; gas samples obtained after steady state achieved (2.5hrs) indicated by stable voltage 
As the CO faradaic efficiency seemed to plateau around $78-80 \%$, an operating condition of $200 \mathrm{~mA} / \mathrm{cm}^{2}$ was chosen as it produced viable CO production rates for bioconversion [144]. A series of test were then conducted by controlling the maximum voltage of the electrolysis cell while operating at constant current [Fig. 24]. It was then determined that a minimum voltage of $3.2 \mathrm{~V}$ was necessary to maintain the $\mathrm{CO}_{2}$ electrolysis at $200 \mathrm{~mA} / \mathrm{cm}^{2}$.

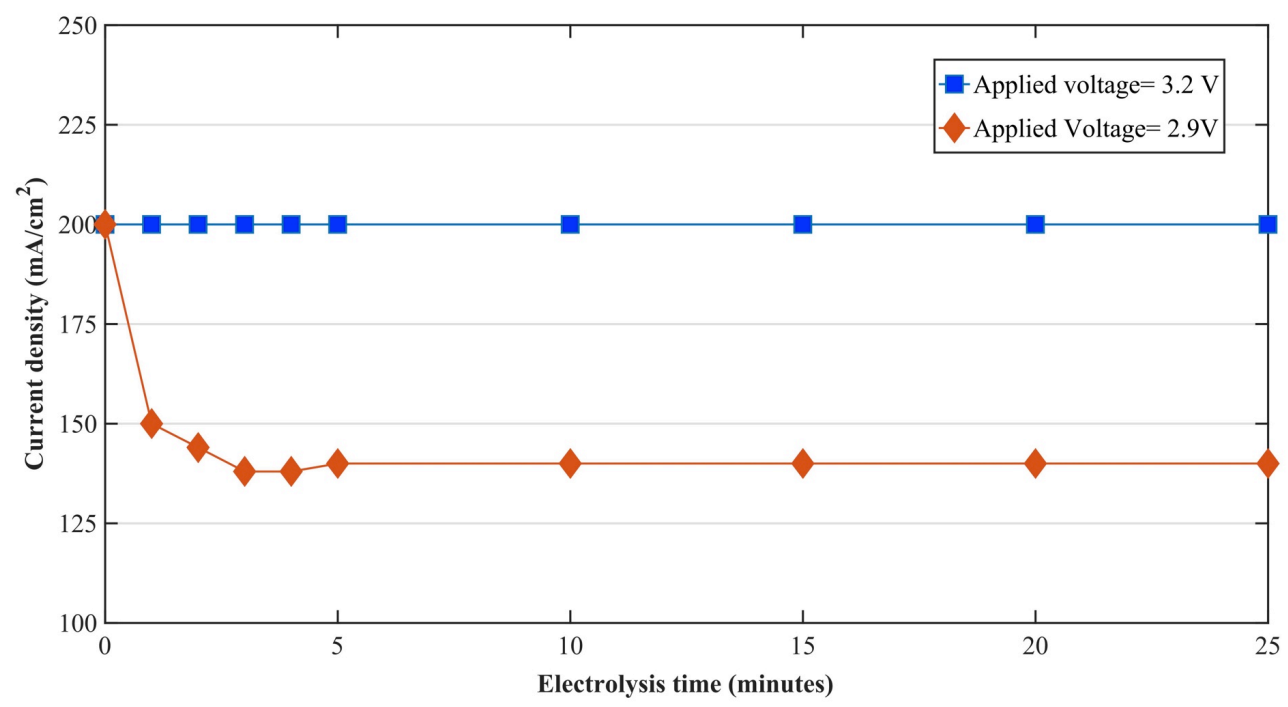

Figure 24: $\mathrm{CO}_{2}$ electrolysis current density vs. time- $\mathrm{CO}_{2}$ electrolysis at $3.2 \mathrm{~V}$ and $2.9 \mathrm{~V}$; Maximum current set to $1000 \mathrm{~mA}, 10 \mathrm{mM} \mathrm{KHCO}_{3}$ electrolyte solution

\subsubsection{Two-stage biohybrid system bioconversion}

Effluent gas from the $\mathrm{CO}_{2}$ electrolysis module was continually bubbled into a downstream bioreactor containing Clostridium ljungdahlii. Throughout fermentations, the $\mathrm{CO}_{2}$ electrolysis voltage was very stable between 3.6-3.8V and maintained a current density of $200 \mathrm{~mA} / \mathrm{cm}^{2}$ [425]. The operating voltage was a bit higher than the voltage $(3.2 \mathrm{~V})$ determined in performance experiments due to increased resistance of the system associated with added pressure from the 
bioreactor attachment. As a baseline for performance, the system was run under mixotrophic conditions $(5 \mathrm{~g} / \mathrm{L}$ fructose added and substantial growth was observed after 24 hours as dry cell densities approached $1.3 \mathrm{~g} / \mathrm{L}$ [Fig. 25]. Under autotrophic conditions ( $0 \mathrm{~g} / \mathrm{L}$ fructose added), cell densities were about $0.4 \mathrm{~g} / \mathrm{L}$ after 24 hours of culturing. This is expected as much more reducing power is available under mixotrophic conditions leading to higher cell densities. Under autotrophic conditions, cells continued to slowly grow and after 120 hours of culturing reached a cell density of $0.54 \mathrm{~g} / \mathrm{L}$.
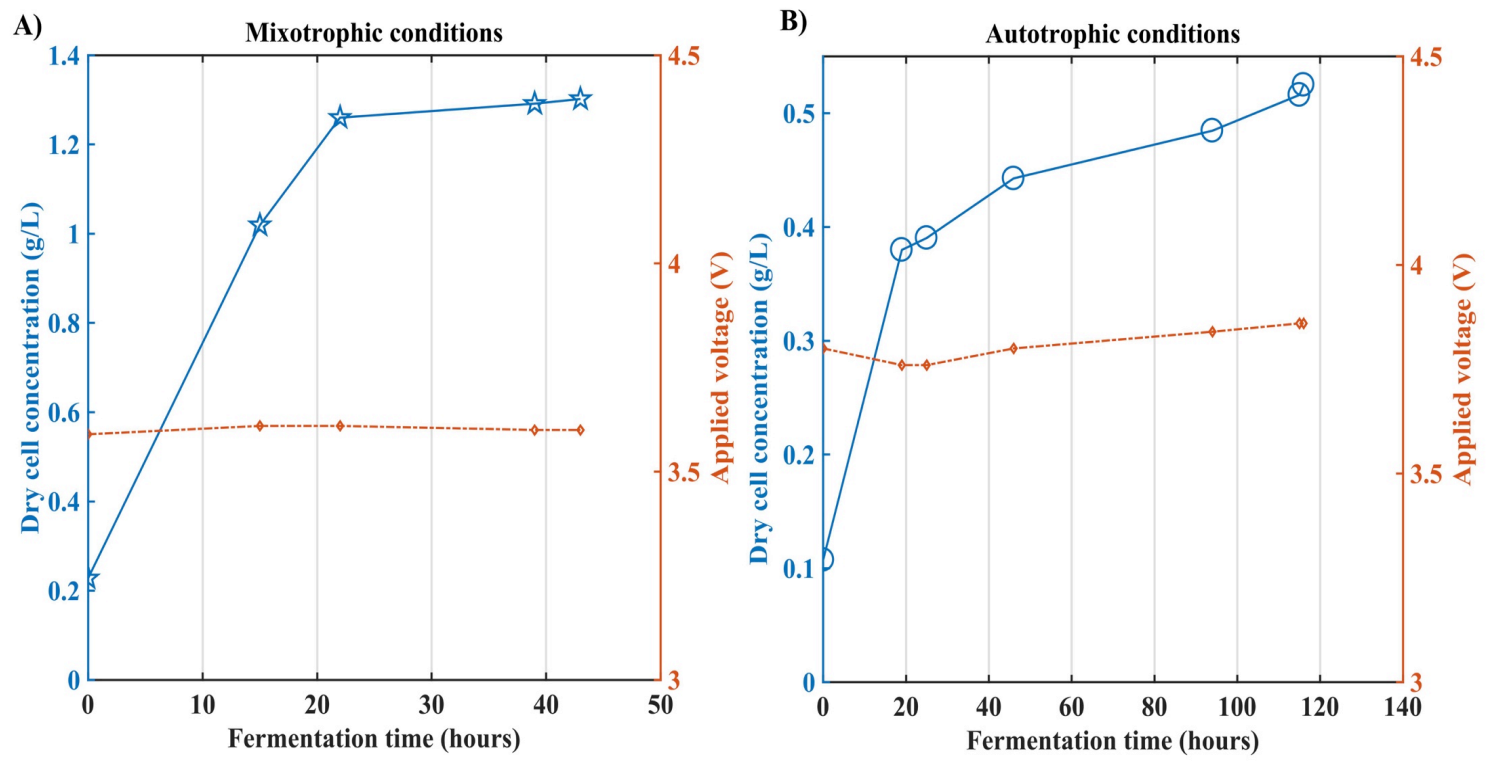

Figure 25: Biohybrid system microbial growth and system stability-C. ljungdahlii cultured with $\mathrm{CO}_{2}$ electrolysis conducted at $200 \mathrm{~mA} / \mathrm{cm}^{2}$; microbes grown with media amended with fructose A) and without B) Dry cell concentration determined from optical density (600nm)

Acetate and ethanol were the major products under both mixotrophic and autotrophic conditions. After 48 hours of culturing, autotrophic fermentations yielded approximately $3.0 \mathrm{~g} / \mathrm{L}$ and 28 $\mathrm{mg} / \mathrm{L}$ of acetate and ethanol, respectively [Fig. 26]. As expected, mixotrophic fermentations achieved around $3.4 \mathrm{~g} / \mathrm{L}$ and $153 \mathrm{mg} / \mathrm{L}$ more acetate and ethanol due to great availability of substrates. This computed to a rate of ca. $2.7 \mathrm{~g} / \mathrm{l} / \mathrm{day}$ of acetate and $100 \mathrm{mg} / \mathrm{l} / \mathrm{day}$ of ethanol. 
Interestingly, the rate of acetate production under autotrophic conditions was comparable reaching around $1.5 \mathrm{~g} / \mathrm{l} / \mathrm{day}$. It is worth noting that this acetate production rate is a significant improvement compared to state-of-the-art MES designs [19]. Ethanol production rate was around $14 \mathrm{mg} / \mathrm{l} / \mathrm{day}$ but as this metabolite is associated with the non-growth phase it was likely much higher between later time points (i.e. between 48-120 hours).
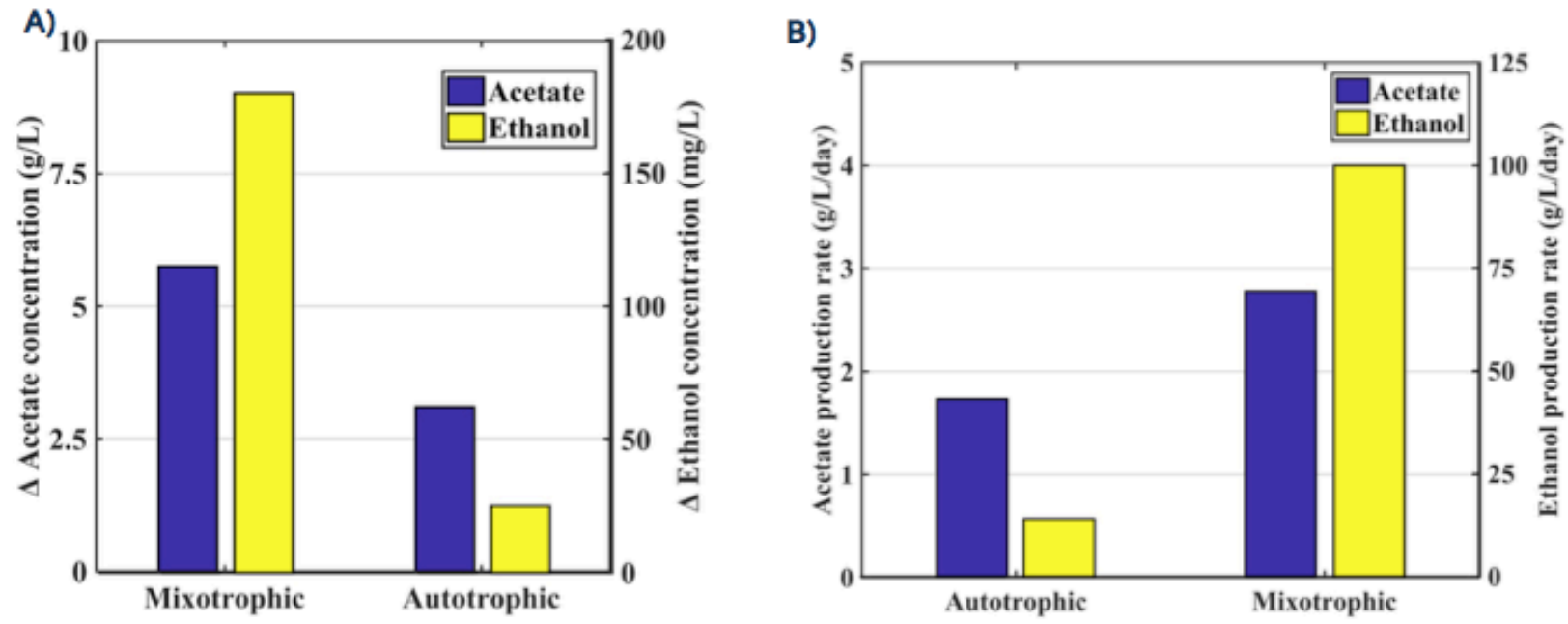

Figure 26: C.ljungdahlii acetate (left axis) and ethanol production (right axis) in two-stage biohybrid system over $48 \mathrm{hrs}$ - Electrolysis at constant current of $200 \mathrm{~mA} / \mathrm{cm}^{2}$. ljungdahlii acetate (left axis) and ethanol production (right axis) rate comparison in two-stage biohybrid system over $48 \mathrm{hrs}$; Electrolysis at constant current of $200 \mathrm{~mA} / \mathrm{cm}^{2}$

\subsubsection{C.ljungdahlii strain optimization for enhanced ethanol production}

To improve the ethanol production rate and yield of C.ljungdahlii a mutant strain was developed using a Crisper-Cas 9 deletion. Wild-type and PtA mutant C.ljungdahlii strains were cultured with $\mathrm{H}_{2} / \mathrm{CO}_{2}$ and fructose $(5 \mathrm{~g} / \mathrm{l})$ to quantify the impact of the deletion. PtA mutant cultures produced $80 \mathrm{mg} / \mathrm{g}$-cell more ethanol than the wild type strain [Fig. 27]. In addition the molar ethanol to acetate ratio was $26 \%$ higher (.11 vs. 09). As the PtA enzyme is directly linked to growth via the Wood-ljungdahl pathway, it is expected that this effect will be exacerbated in 
cultures maintained under syngas alone (without fructose addition). Future characterization work with this PtA mutant is currently underway.

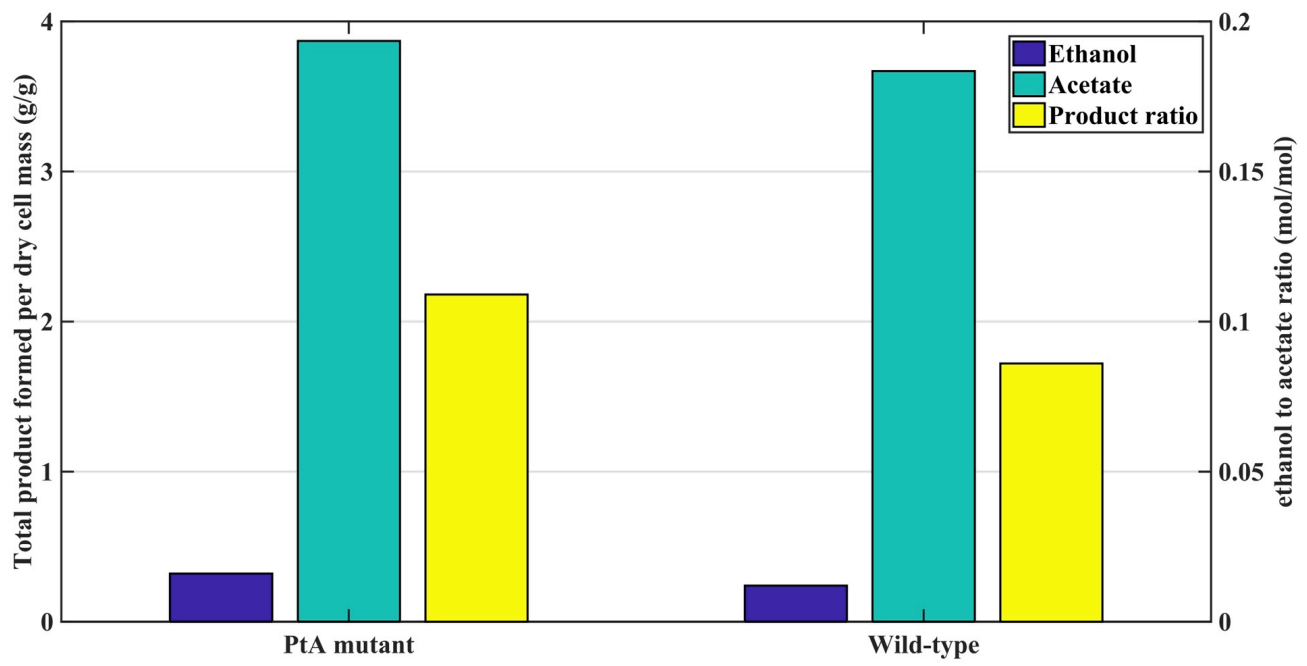

Figure 27: Comparison of C.ljungdahlii PtA mutant and wild-type control product generationproducts generated per dry cell weight (left axis); molar product ratio (right axis)

\section{Conclusions}

A novel two-stage biohybrid system for $\mathrm{CO}_{2}$ conversion into carbon commodities has been developed. The $\mathrm{CO}_{2}$ gas diffusion electrolyser showed excellent stability at a high current density of $200 \mathrm{~mA} / \mathrm{cm}^{2}$ and produced $\mathrm{CO}$ at an outstanding faradaic efficiency of over $80 \%$. This yielded a $\mathrm{CO}$ production rate of $15 \mathrm{mmol} / \mathrm{hr}$. When paired with a bioreactor containing a pure culture of C.ljungdhalii, exceptional cell densities of $1.3 \mathrm{~g} / \mathrm{L}$ and $0.44 \mathrm{~g} / \mathrm{L}$ where achieved after 48 hours of mixotrophic and autotrophic culturing, respectively. This amounted to high acetate and ethanol production rates of $1.5 \mathrm{~g} / \mathrm{l} /$ day and $14 \mathrm{mg} / \mathrm{l} /$ day under autotrophic conditions. Work with PtA mutant strains of C.ljungdahlii showed great potential to improved ethanol production as 80 more $\mathrm{mg}$ ethanol/g cell were achieved compared to the wild-type. Further optimization of the biohbyrid system are on-going including exploration of ways to increase $\mathrm{CO}$ generation rates, 
enhance mass transfer in the bioreactor and refine the C.ljungdhalii mutant strains towards desired products. 


\section{CHAPTER 6}

\section{FUTURE RESEARCH}

\subsection{Platinum nanoparticles dispersed in novel covalent organic frameworks for electrochemical hydrogen production and conversion}

\subsubsection{Introduction}

The portfolio of compounds that can be electrochemically generated and used as electron carriers in a biohybrid system is presently very limited. Currently the only viable candidates include compounds such as $\mathrm{H}_{2}, \mathrm{CO}$ and formate that can be produced at high faradaic efficiencies and current densities $[148,149]$. It is not a surprise that each of these compounds only requires a two electrons transfer as electroreduction of $\mathrm{CO}_{2}$ involving greater than two electrons has been a tremendous challenge due to a lack of product selectivity and low energetic efficiency [150]. Breakthroughs in direct multi-electron $\mathrm{CO}_{2}$ reduction could allow for high efficiency generation of biocompatible electron carriers such as methanol, methane, ethylene, and acetate, in-turn opening the door to new types of microbes that can be integrated into biohybrid systems and products that can be synthesized. Catalytic hydrogenation provides an alternative to direct electrochemical $\mathrm{CO}_{2}$ reduction to efficiently synthesize new feedstock compounds for biohybrid conversion. In catalytic hydrogenation processes, electrochemically produced hydrogen is reacted with $\mathrm{CO}_{2}$ to produce chemicals such as $\mathrm{CO}$, methane, methanol, or ammonia (via haberbosch process with nitrogen) [151-153]. Recently, this field has gained a lot of momentum and the production of methane and methanol using catalytic hydrogenation has already reached energetic efficiencies up to $70 \%[154,155]$.

The key to the overall efficiencies of these processes, however, lies in the efficiency of the primary hydrogen evolution reaction $\left(\mathrm{HER}, 2 \mathrm{H}^{+}+2 \mathrm{e}^{-} \rightarrow \mathrm{H}_{2}\right.$ ). HER is sluggish and requires 
electrocatalysts to achieve efficient water splitting [156-158]. Platinum (Pt) is the most wellknown and efficient electrocatalyst that outperforms many other noble or non-noble metals. Recently, it was found that dispersing ultrafine Pt nanoparticles (PtNPs) in a carbon matrix could significantly improve the catalytic capability and the stability of the catalysts [159]. Various supporting substrates have been utilized to increase the dispersion of metal-nanoparticles, including carbon substrate, graphene, porous polymers, mesoporous silica, covalent organic frameworks (COFs), and metal-organic frameworks (MOFs) [159-167]. Amongst these, COFs are promising templates to prepare nanocomposites because of their high porosity, crystallinity, stability, and functional group diversity [159, 168-171]. In this work, a novel porphyrinbipyridine-based COF (COF-bbyTPP) was synthesized and PtNPs were well dispersed within its matrix. The as-prepared materials were characterized using a suite of tools including Fourier transform infrared resonance (FTIR), nuclear magnetic resonance (NMR), Brunauer-EmmettTeller analysis (BET), inductively coupled plasma mass spectrometry (ICP-MS), transmission electron microscopy (TEM), and X-ray photoelectron spectroscopy (XPS). The electrochemical performance of these catalysts was then evaluated using cyclic voltammetry and controlled potential water electrolysis. Future research will focus on optimization of the HER reaction using these catalysts and integration of the electrochemically produced $\mathrm{H}_{2}$ for catalytic hydrogenation of intermediates to generate feedstock compounds for bioconversion.

\subsubsection{Material and methods}

\section{Synthesis of COF-bpyTPP}

A pyrex glass tube was charged with porphyrin tetraamine $(20 \mathrm{mg}, 0.0296 \mathrm{mmol})$ and bipyridine-dialdehyde $(16 \mathrm{mg}, \quad 0.074 \mathrm{mmol})$. A mixture of solvents, butanol/1,2- 
dichlorobenzene/6M acetic acid (1/1/0.25, total volume $2.25 \mathrm{~mL})$ was added. The glass tube was then frozen at $77 \mathrm{deg} . \mathrm{K}$ and sealed under vacuum using an open flame. It was then placed in an oven at $120^{\circ} \mathrm{C}$ for 3 days. Violet precipitates were formed, which were collected, washed with butanol and acetone, and dried to give the product $17.0 \mathrm{mg}(80 \%)$.

\section{Synthesis of Pt@COF-bpyTPP}

COF-bpy-TPP (4 mg) was dispersed in methanol (3 mL). An aqueous solution of $\mathrm{K}_{2} \mathrm{PtCl}_{4}(5 \mathrm{mg}$, $2 \mathrm{~mL}$ ) was added to the above COF suspension. After removing the solvents, the remaining solid was re-dispersed in $\mathrm{MeOH} / \mathrm{H}_{2} \mathrm{O}(5 \mathrm{~mL}, \mathrm{v} / \mathrm{v}=3: 2)$. A solution of sodium borohydride in methanol (0.25 M, $2 \mathrm{~mL})$ was added. The mixture was stirred at room temperature for 2 days. The solid was collected, washed with ethanol and dichloromethane, and dried to give the product (3.2 $\mathrm{mg}$ ).

\section{Material characterization}

${ }^{1} \mathrm{H}$ NMR spectra were recorded on a Bruker Avance-III 300 NMR spectrometer. FTIR spectra were measured with a Agilent Technologies, Cary 630. $\mathrm{N}_{2}$ adsoprtion isotherm was measured by a Quantachrome Autosorb ASiQ automated gas sorption analyzer. TEM was performed on a FEI Tecnai T12, 120kV TEM. ICP-MS was recorded on a Perkin Elmer Optima 7300DV. XPS was measured by PE-5800 X-ray photoelectron spectroscopy.

\subsubsection{Electrochemical HER performance}

A mixture of $10 \mathrm{mg}$ of catalyst and $10 \mathrm{mg}$ of carbon black was sonicated for $30 \mathrm{~min}$ in $4 \mathrm{~mL}$ of DMF. $100 \mu \mathrm{L}$ of the resulting suspension was then mixed with $5 \%$ nafion $(1: 100)$, drop casted on two sides of carbon paper (Toray-50\% wet-proofed) and dried for 48 hours at room temperature 
$\left(25 \pm 0.1^{\circ} \mathrm{C}\right)$ in a laminar flow hood. Cyclic voltammetry and controlled potential electrolysis were conducted in a Pine Research (WaveDriver- 200 series) potentiostat in combination with a standard Pine Research electrochemical cell (AKCELL1) containing two chambers separated by a glass frit. The cathodic compartment contained catalysts coated-carbon paper as the working electrode (partly submerged in the electrolyte solution) and an $\mathrm{Ag} / \mathrm{AgCl}$ reference electrode with ceramic porous frit (BASi-MW-2030). The anodic compartment had a large graphite electrode $\left(\mathrm{OD}=0.250 ", \mathrm{~L}=12^{\prime \prime}\right)$. The supporting electrolyte in both chambers contained $0.5 \mathrm{M} \mathrm{NaHCO}$ dissolved in deionized water. Prior to electrolysis, all electrolytes were saturated with either pure $\mathrm{CO}_{2}$ or argon and the cell was sealed using gas tight rubber seals (Pine Research-14/20; 24/25). Gas samples were obtained directly from the headspace of the electrochemical cell using a $250 \mu \mathrm{l}$ gastight syringe (Hamilton-1700 series) and were immediately analyzed on an Agilent Technologies 7890A gas chromatography system equipped with a thermal conductivity detector (TCD) and packed column. The oven temperature was a constant $60^{\circ} \mathrm{C}$. Injector and detector temperatures were stable at $150^{\circ} \mathrm{C}$ and $250^{\circ} \mathrm{C}$, respectively. Argon gas was used as the carrier

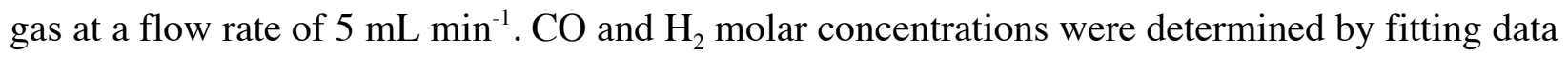
to a five-point standard curve prepared for each gas using standard calibration gases.

\subsubsection{Preliminary results}

\section{Material synthesis and characterization}

Imine-linked COF-bpyTPP was obtained by a Schiff-base reaction between a free-base porphyrin and bipyridine-dialdehyde via an solvothermal synthesis method in a mixed solvent of butanol/o-dichlorobenzene/6M acetic acid [Fig. 28]. The formation of the imine linkages in

COF-bpyTPP were confirmed by FTIR and solid state ${ }^{13} \mathrm{C}$ NMR. For example, FTIR spectra of the COF-bpyTPP showed an absorbance peak around $1650 \mathrm{~cm}^{-1}$ owing to $\mathrm{C}=\mathrm{N}$ bonding in the 
imine linkage [Fig. A.11]. Furthermore, the absence of the $\mathrm{C}=\mathrm{O}$ peak at $1685 \mathrm{~cm}^{-1}$ and $\mathrm{N}-\mathrm{H}$ at $1602 \mathrm{~cm}^{-1}$ indicates that the starting materials were consumed to synthesize the COF material [Fig. A.11]. The presence of imine bonding was further supported by solid state ${ }^{13} \mathrm{C}$ NMR that revealed a characteristic carbon atom peak around about 155 ppm [Fig. A.12, 172].

COF-bpyTPP has metal chelating bipyridine moieties in the backbone of the framework, making it is a good host for nanoparticles [171,173]. The COF-supported PtNPs (PtNPs@COF) were obtained through the initial chelating reaction between COF-bpyTPP and soluble Pt (II) salts, followed by further reduction with $\mathrm{NaBH}_{4}$ to form the metal nanoparticles. Prior to incorporating PtNPs, the BET surface area of the COF-bpyTPP was estimated to be $333 \mathrm{~m}^{2} / \mathrm{g}$.
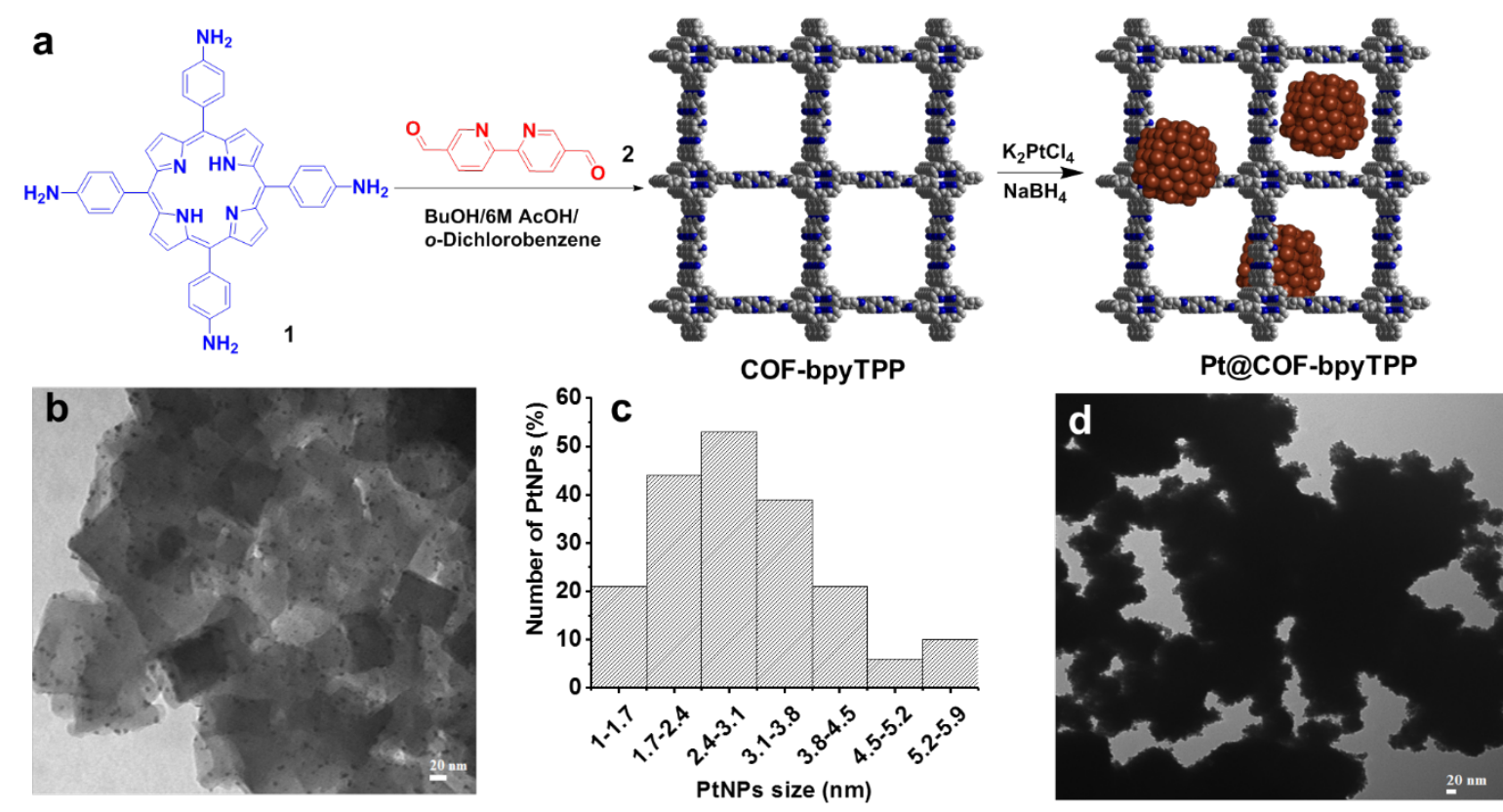

Figure 28: Pt@COF compound and material characterization summary- Schematic representation of preparation of COF-bpyTPP and PtNPs@COF-bpyTPP (a); the TEM image of PtNPs supported on COF-bpyTPP (b) and their size distribution (c), the TEM image of PtNPs prepared in the absence of any $\mathrm{COF}$ materials 
The dispersion and size distribution of the as-prepared PtNPs@COF were assessed by TEM. The TEM images show well-dispersed PtNPs@COF of sizes varying from 1 to $6 \mathrm{~nm}$ [Fig. $28 \mathrm{~b}$, c]. The formation of well-dispersed PtNPs in such small sizes is likely attributed to the presence of the COF support with high porosity and orderly arranged bipyridine metal-chelating sites in the backbone of the framework structures. A control sample (PtNPs without the COF matrix) showed random aggregations of PtNPs indicating the critical role of the COF in the formation of well-dispersed ultrafine nanoparticles [Fig. 28d].

ICP-MS analyses of the PtNPs@COF revealed that the weight percent of Pt loading on the COF materials is $6.09 \mathrm{wt} \%$. The oxidation state of the as-prepared PtNPs was confirmed by XPS measurement [Fig. A.13]. The analysis of the Pt 4f spectrum of the Pt@COF-bpyTPP shows two peaks with binding energies of 71.8 and $74.9 \mathrm{eV}$, corresponding to $4 \mathrm{f}_{7 / 2}$ and $4 \mathrm{f}_{5 / 2}$ of $\mathrm{Pt}^{0}$.

\section{Electrochemical HER performance evaluation}

The catalytic activity and stability of PtNPs@COF were evaluated under alkaline conditions.

It is worthy pointing out that HER in alkaline solution is more difficult than acidic conditions as water must first be dissociated by the Volmer step to make adsorbed hydrogen on the surface of metals, leading to a higher overpotential [174]. As such, catalyst testing under alkaline conditions is a truer test of HER performance as under acidic conditions HER can occur at almost no overpotential via proton reduction [174]. Furthermore, testing under a higher $\mathrm{pH}$ is far more practical for industrial applications of water electrolyzers at large-scale [174]. Cyclic voltammetry $(\mathrm{CV})$ was conducted at $1 \mathrm{mV} / \mathrm{s}$ with $0.5 \mathrm{M} \mathrm{NaHCO}_{3}$ dissolved in deionized water, saturated with $\mathrm{CO}_{2}(\mathrm{pH} 8.3) . \mathrm{CV}$ results revealed that the onset potential of $\mathrm{Pt} / \mathrm{C}$ was ca. $-500 \mathrm{mV}$ more negative than that of PtNPs@COF [Fig. 29a]. This was attributed to an improved kinetics barrier in the water dissociation step by the as prepared PtNPs@COF catalyst. To clearly 
demonstrate the HER capabilities of the compounds, controlled potential electrolysis (-0.95 V vs. $\mathrm{Ag} / \mathrm{AgCl})$ were conducted for one hour [Fig. 29]. The reduction current of PtNPs@COF was very stable for over an hour of electrolysis and achieved a high current density of $-70 \mathrm{~mA} / \mathrm{cm}^{2}$. This demonstrated excellent performance compared to the $\mathrm{Pt} / \mathrm{C}$ that showed signs of slow decomposition over the electrolysis and achieved a current density of only $-10 \mathrm{~mA} / \mathrm{cm}^{2}$ under the same conditions [Fig. 29b]. These differences in current densities were also reflected in the amounts of hydrogen evolved at each catalyst. After one-hour ca. $490 \mu \mathrm{mol} / \mathrm{mg}$ of hydrogen gas was produced by the electrocatalysis using PtNPs@COF (6.09 wt\% Pt). This amount is 13 times larger than that was produced in the presence of $\mathrm{Pt} / \mathrm{C}(10 \mathrm{wt} \% \mathrm{Pt}, 38 \mu \mathrm{mol} / \mathrm{mg})$ [Fig. 29c]. TEM image of PtNPs@COF after the electrolysis shows no signs of agglomeration of the nanoparticles, indicating its high stability under alkaline electrolysis conditions [Fig. 29d]. The excellent catalytic performance can be attributed to the porous structure of the COF-bpyTPP and the dispersity of PtNPs on the COF substrate. 

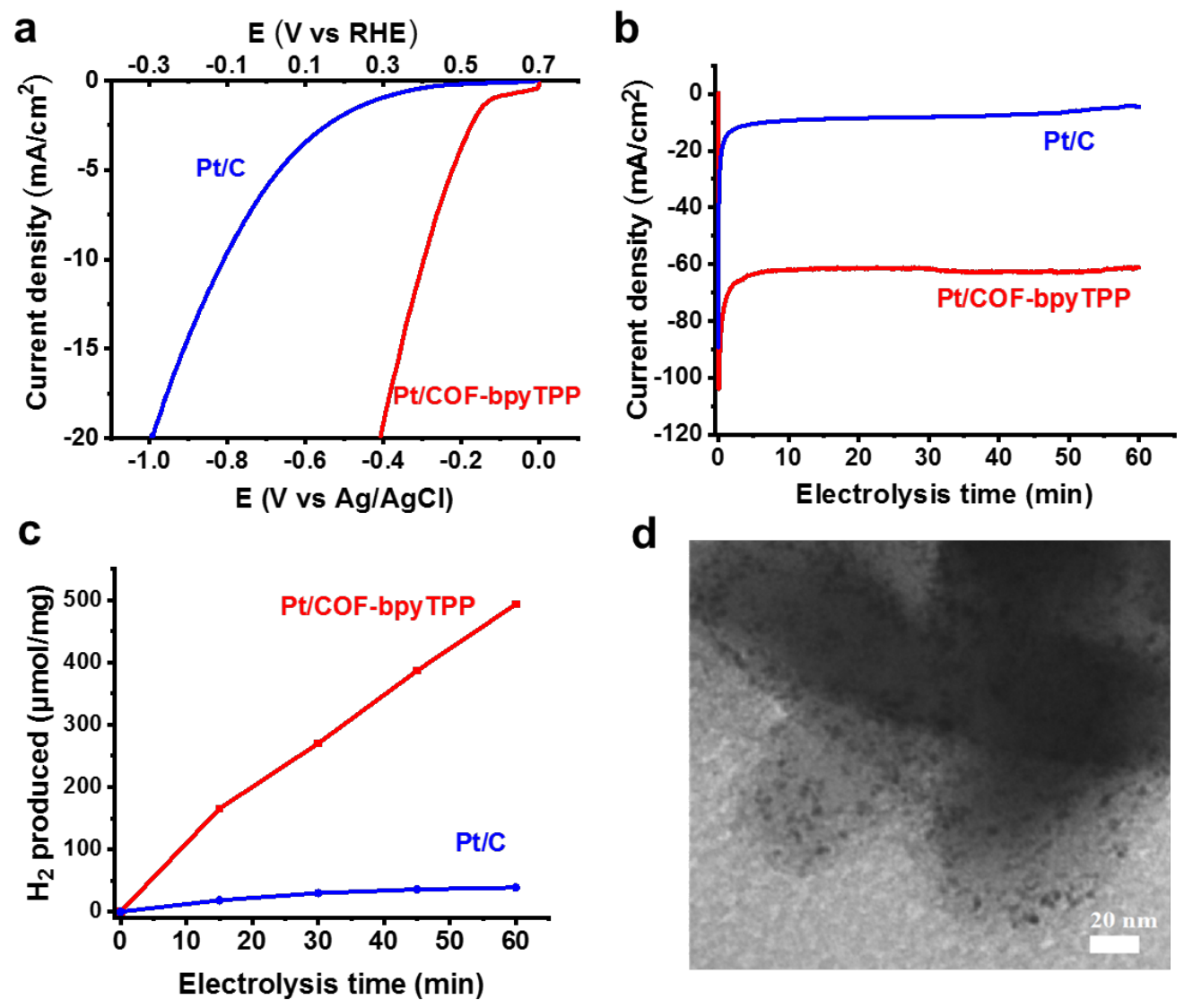

Figure 29: Pt@COF electrochemical hydrogen evolution summary (a) Polarization curves of PtNPs@COF (red line) and Pt/C (blue line) in a $0.5 \mathrm{M} \mathrm{NaHCO}_{3}$ at a $100 \mathrm{mVs}^{-1}$ scan rate; (b) Controlled potential measurements at $-0.95 \mathrm{~V}$ vs. $\mathrm{Ag} / \mathrm{AgCl}$ for 60 minutes with PtNPs@COF (red line) and Pt/C (blue line); (c) The amount of hydrogen evolution by PtNPs@COF (red rectangular) and Pt/C (blue circle); (d) A TEM image of PtNPs@COF after one-hour bulk electrolysis under the alkaline condition

\section{On-going and future work}

The as-synthesized COF-bpyTPP are an excellent supporting material to deposit metal nanoparticles because of their high crystallinity, large porosity, and thermal stability in addition to ample nucleation sites. COF-bpyTPP successfully supported PtNPs $(\sim 2.95 \mathrm{~nm})$ which were highly active and stable. The obtained PtNPs@COF shows the enhanced catalytic performance and excellent stability toward HER in alkaline media. PtNPs@COF outperformed commercial $\mathrm{Pt} / \mathrm{C}$ in alkaline solution, generating 13 times more hydrogen with nearly $100 \%$ faradaic 
efficiencies. Future work will focus on further characterizing the HER potential of this catalyst under different bulk electrolyte $\mathrm{pH}$ as well as in the presence of different solvation cations. Furthermore, this catalyst may be integrated into a new biohybrid system to generate $\mathrm{H}_{2}$ that is catalytically hydrogenated to a product such as methanol for microbial conversion. More applications of this catalyst are still being explored.

\subsection{Functionalized hollow fiber membrane for dual electrochemical $\mathrm{CO}_{2}$ reduction and enhanced gas mass transfer}

The bottleneck of syngas fermentation processes has often been the gas-liquid mass transfer of gaseous substrates $\left(\mathrm{H}_{2} / \mathrm{CO} / \mathrm{CO}_{2}\right)$ to the cell surface. A variety of reactor types including stirred tank reactors (STR), trickle bed reactors (TBR), packed bed reactors (PBR), monolithic biofilm reactors (MLBR), membrane-based reactors (MBR), and bubble column reactors (BCR) have been tested [175]. Orgill et al. (2013) compared the volumetric mass transfer coefficient $\left(\mathrm{K}_{\text {tot }} \mathrm{A} / \mathrm{V}_{\mathrm{L}}\right)$ in 3 syngas fermentation configurations, and they found hollow fiber membrane reactors (HFMR) provided the highest $\mathrm{K}_{\text {tot }} \mathrm{A} / \mathrm{V}_{\mathrm{L}}\left(1062 \mathrm{~h}^{-1}\right)$ (more than doubled TBR $\left(421 \mathrm{~h}^{-1}\right)$ and nearly 10 -folds higher than STR $\left.\left(114 \mathrm{~h}^{-1}\right)\right)[176]$.

Therefore, future research will aim to maximize syngas mass transfer and uptake by combing gas-delivery and utilization to a single chamber using an innovative HFMs coated with electro-catalysts for $\mathrm{CO}_{2}$ conversion. The innovative catalyst coated HFMs will serve dual roles as both the site of $\mathrm{CO}_{2}$ conversion and syngas dispersion into the surrounding media for bacterial uptake. This will greatly simplify the reactor design (as well as lower volumetric requirements) and allow for more rapid generation of desired products. The $\mathrm{CO}_{2}$ feedstock will be fed directly through HFMs to the growth media of the bioreactor and will be converted to syngas via an electrocatalyst on the surface of the HFMs [Fig. 30]. 
The functionalized-HFMs will be constructed using coating methods similar to those used in Chapter 4. HFMs will then be characterized using techniques such as scanning electron microscopy (SEM), X-ray diffraction (XRD), X-ray photo-electron spectroscopy (XPS) and insitu raman spectroscopy. The electrocatalyic capabilities of the functionalized HFMs will then quantified using electrochemical techniques such as CV, linear sweep voltammetry (LSV), electrochemical impedance spectroscopy (EIS). $\mathrm{CO}_{2}$ conversion products will be defined under controlled current experiments. The functionalized HFM will then be optimized for the desired $\mathrm{CO} / \mathrm{H}_{2}$ composition for fermentation by adjusting parameters such as applied potential, $\mathrm{CO}_{2}$ feed rate and operating temperature.

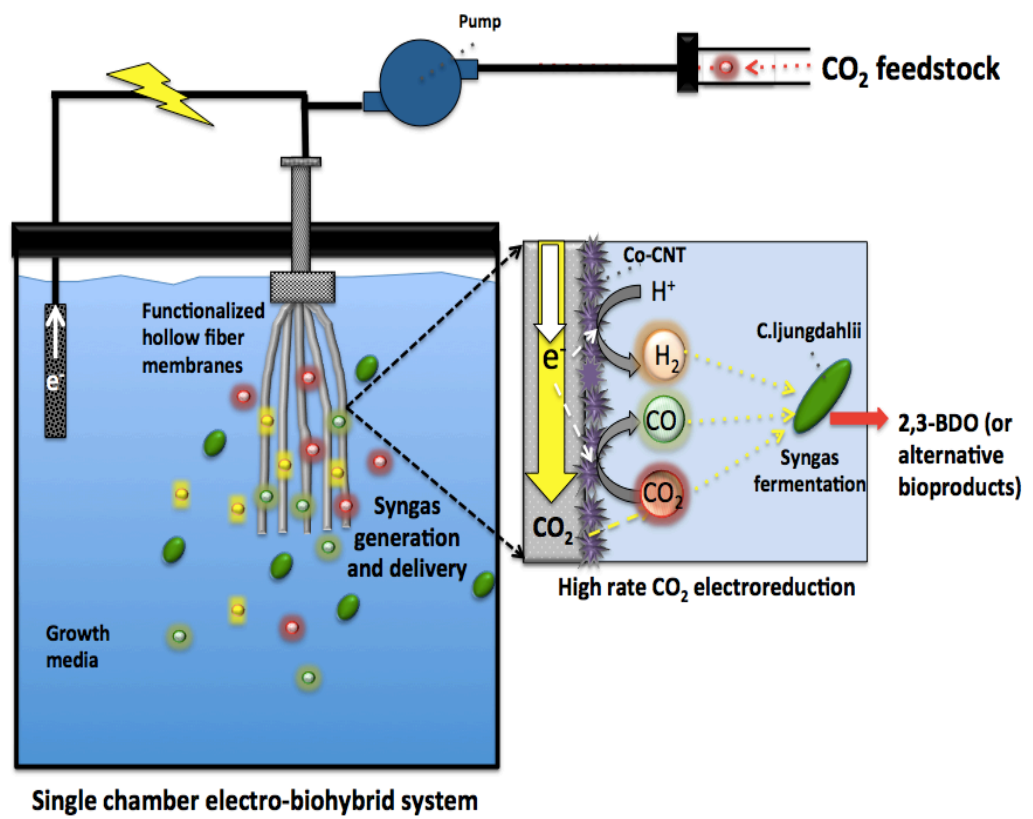

Figure 30: One-pot biohybrid system schematic -Single chamber reactor with functionalized HFM for simultaneous syngas generation and utilization; Feed $\mathrm{CO} 2$ is electrochemically converted to syngas that is available for microbial uptake in the same reactor 


\section{REFERENCES}

[1] "Carbon Dioxide Concentration I NASA Global Climate Change." NASA, NASA, 15 May 2019, climate.nasa.gov/vital-signs/carbon-dioxide/.

[2] Team, ESRL Web. "ESRL Global Monitoring Division - Global Greenhouse Gas Reference Network." NOAA Earth System Research Laboratory, 1 Oct. 2005, www.esrl.noaa.gov/gmd/ccgg/trends/global.html.

[3] "World Population Projected to Reach 9.8 Billion in 2050, and 11.2 Billion in 2100 I UN DESA Department of Economic and Social Affairs." United Nations, United Nations, www.un.org/development/desa/en/news/population/world-population-prospects-2017.html.

[4] Jossi, Frank. "An FAQ on 45Q: What Federal Carbon Storage Tax Credit Means for Midwest." Energy News Network, 9 July 2018, energynews.us/2018/07/10/midwest/an-faq-on45q-what-federal-carbon-storage-tax-credit-means-for-midwest/.

[5] Mohammadi, Maedeh, et al. "Bioconversion of synthesis gas to second generation biofuels: a review." Renewable and Sustainable Energy Reviews 15.9 (2011): 4255-4273.

[6] Blankenship, Robert E., et al. "Comparing photosynthetic and photovoltaic efficiencies and recognizing the potential for improvement." science 332.6031 (2011): 805-809.

[7] Cotton, Charles AR, et al. "Photosynthetic constraints on fuel from microbes." Frontiers in bioengineering and biotechnology3 (2015): 36.

[8] Claassens, Nico J., et al. "Harnessing the power of microbial autotrophy." Nature Reviews Microbiology 14.11 (2016): 692.

[9] Zhu, Xin-Guang, Stephen P. Long, and Donald R. Ort. "What is the maximum efficiency with which photosynthesis can convert solar energy into biomass?." Current opinion in biotechnology 19.2 (2008): 153-159.

[10] Bushuyev, Oleksandr S., et al. "What should we make with $\mathrm{CO} 2$ and how can we make it?." Joule 2.5 (2018): 825-832.

[11] Xie, Huan, et al. "Recent advances in Cu-based nanocomposite photocatalysts for $\mathrm{CO} 2$ conversion to solar fuels." Journal of energy chemistry 26.6 (2017): 1039-1049.

[12] Duan, Xiaochuan, et al. "Metal-free carbon materials for $\mathrm{CO} 2$ electrochemical reduction." Advanced Materials 29.41 (2017): 1701784.

[13] Peng, Xiong, Stavros G. Karakalos, and William E. Mustain. "Preferentially oriented Ag nanocrystals with extremely high activity and faradaic efficiency for $\mathrm{CO} 2$ electrochemical reduction to CO." ACS applied materials \& interfaces 10.2 (2018): 1734-1742. 
[14] Jeon, Hyo Sang, et al. "Prism-shaped $\mathrm{Cu}$ nanocatalysts for electrochemical CO2 reduction to ethylene." ACS Catalysis8.1 (2017): 531-535.

[15] Cook, Brian J., et al. "Countercations and Solvent Influence CO2 Reduction to Oxalate by Chalcogen-Bridged Tricopper Cyclophanates." Journal of the American Chemical Society140.17 (2018): 5696-5700.

[16] Shen, Jing, et al. "DFT study on the mechanism of the electrochemical reduction of $\mathrm{CO} 2$ catalyzed by cobalt porphyrins." The Journal of Physical Chemistry C 120.29 (2016): 1571415721 .

[17] Jouny, Matthew, Wesley W. Luc, and Feng Jiao. "A General Techno-Economic Analysis of CO2 Electrolysis Systems." Industrial \& Engineering Chemistry Research (2018).

[18] Hori, Yoshio, Katsuhei Kikuchi, and Shin Suzuki. "Production of CO and CH4 in electrochemical reduction of $\mathrm{CO} 2$ at metal electrodes in aqueous hydrogencarbonate solution." Chemistry Letters 14.11 (1985): 1695-1698.

[19] Jiang, Yong, et al. "Carbon dioxide and organic waste valorization by microbial electrosynthesis and electro-fermentation." Water research (2018).

[20] Rabaey, Korneel, and René A. Rozendal. "Microbial electrosynthesis-revisiting the electrical route for microbial production." Nature Reviews Microbiology 8.10 (2010): 706.

[21] Lovley, Derek R., and Kelly P. Nevin. "Electrobiocommodities: powering microbial production of fuels and commodity chemicals from carbon dioxide with electricity." Current opinion in biotechnology 24.3 (2013): 385-390..

[22] LaBelle, Edward V., and Harold D. May. "Energy Efficiency and Productivity Enhancement of Microbial Electrosynthesis of Acetate." Frontiers in microbiology 8 (2017): 756.

[23] Tremblay, Pier-Luc, Largus T. Angenent, and Tian Zhang. "Extracellular electron uptake: among autotrophs and mediated by surfaces." Trends in biotechnology 35.4 (2017): 360-371.

[24] Celińska, E., and W. Grajek. Biotechnological production of 2, 3-butanediol-current state and prospects. Biotechnology advances. (2009);27:715-725.

[25] Klasson, K. Thomas, et al. Bioconversion of synthesis gas into liquid or gaseous fuels. Enzyme and Microbial Technology. (1992);14:602-608

[26] Munasinghe et al.. "Biomass-derived syngas fermentation into biofuels: opportunities and challenges." Bioresource technology 101.13 (2010): 5013-5022

[27] Ren, Z., et al. Characterization of the cellulolytic and hydrogen-producing activities of six mesophilic Clostridium species. Journal of applied microbiology 103.6 (2007): 2258-2266. 
[28] Younesi, H. et al. Ethanol and acetate production from synthesis gas via fermentation processes using anaerobic bacterium, Clostridium ljungdahlii. Biochemical Engineering Journal. (2005); 27.2:110-119.

[29] Drake HL, Gößner AS, Daniel SL: Old Acetogens, New Light. Annals of the New York Academy of Sciences. (2008); 1125:100-128.

[30] Ragsdale, Stephen W. Life with carbon monoxide. Critical reviews in biochemistry and molecular biology. (2004).39.3:165-195.

[31] Renna, Maria Catherine, et al. Regulation of the Bacillus subtilis alsS, alsD, and alsR genes involved in post-exponential-phase production of acetoin. Journal of Bacteriology. (1993);175.12:3863-3875.

[32] Phillips, John R., Edgar C. Clausen, and James L. Gaddy. "Synthesis gas as substrate for the biological production of fuels and chemicals." Applied biochemistry and biotechnology 45.1 (1994): 145-157.

[33] Sander, Rolf. Compilation of Henry's law constants for inorganic and organic species of potential importance in environmental chemistry.(1999); 107.

[34] "Gasification Introduction-Range of Syngas Compositions Across Different Gasifier Type, and Feedstock Produced by the Gasification of Coal Feedstocks." National Energy Technology Laboratory. Department of Energy, n.d. Web. 11 Apr. (2017).

[35] Ciferno, Jared P., and John J. Marano. Benchmarking biomass gasification technologies for fuels, chemicals and hydrogen production. US Department of Energy. National Energy Technology Laboratory. (2002).

[36] Cotter, Jacqueline L., Mari S. Chinn, and Amy M. Grunden.Influence of process parameters on growth of Clostridium ljungdahlii and Clostridium autoethanogenum on synthesis gas.Enzyme and Microbial Technology. (2009);44: 281-288.

[37] Gaddy JL, Clausen EC: Clostridium ljungdahlii, an anaerobic ethanol and acetate producing microorganism .(1992).

[38] Gaddy JL: Biological production of ethanol from waste gases with Clostridium ljungdahlii. (2000).

[39] Kim, Young-Kee, et al. Enhancement of bioethanol production in syngas fermentation with Clostridium ljungdahlii using nanoparticles. Bioresource technology. (2014);159:446-450.

[40] Klasson, K. T., et al. Biological conversion of coal and coal-derived synthesis gas. Fuel. (1993);72:1673-1678. 
[41] Mohammadi, Maedeh, et al. Bioconversion of synthesis gas to second generation biofuels: a review. Renewable and Sustainable Energy Reviews. (2011);15:4255-4273.

[42] Mohammadi, Maedeh, et al. Sustainable ethanol fermentation from synthesis gas by Clostridium ljungdahlii in a continuous stirred tank bioreactor. Journal of Chemical Technology and Biotechnology. (2012);87:837-843.

20[43] Mohammadi, M., et al. Clostridium ljungdahlii for production of biofuel from synthesis gas. Energy Sources, Part A: Recovery, Utilization, and Environmental Effects. (2016); 38: 427434.

[44] Phillips, J. R., et al. Biological production of ethanol from coal synthesis gas. Applied biochemistry and biotechnology. (1993);39:559-571.

[45] Rajagopalan, Srini, Rohit P. Datar, and Randy S. Lewis. Formation of ethanol from carbon monoxide via a new microbial catalyst. Biomass and Bioenergy. (2002);23:487-493.

[46] Xiu, Zhi-Long, and An-Ping Zeng. Present state and perspective of downstream processing of biologically produced 1,3-propanediol and 2, 3-butanediol. Applied Microbiology and Biotechnology. (2008);78:917-926.

[47] Heiskanen, Harri, Ilkka Virkajärvi, and Liisa Viikari. "The effect of syngas composition on the growth and product formation of Butyribacterium methylotrophicum." Enzyme and Microbial Technology 41.3 (2007): 362-367.and Microbial Technology 41.3 (2007): 362-367.

[48] Köpke, Michael, et al. 2, 3-Butanediol production by acetogenic bacteria, an alternative route to chemical synthesis, using industrial waste gas. Applied and environmental microbiology. (2011);77: 5467-5475.

[49] Younesi, Habibollah, Ghasem Najafpour, and Abdul Rahman Mohamed. Liquid fuel production from synthesis gas via fermentation process in a continuous tank bioreactor (CSTBR) using Clostridium ljungdahlii. Iran J Biotechnol. (2006); 4:45-53

[50] Chookaew, Teera, Poonsuk Prasertsan, and Zhiyong Jason Ren. "Two-stage conversion of crude glycerol to energy using dark fermentation linked with microbial fuel cell or microbial electrolysis cell." New biotechnology 31.2 (2014): 179-184.

[51] Singer, Scott, et al. "Anaerobic membrane gas extraction facilitates thermophilic hydrogen production from Clostridium thermocellum." Environmental Science: Water Research \& Technology (2018).

[52] Grimalt-Alemany, Antonio, et al. "Enrichment of syngas-converting mixed microbial consortia for ethanol production and thermodynamics-based design of enrichment strategies." Biotechnology for biofuels 11.1 (2018): 198. 
[53] Esquivel-Elizondo, Sofia, et al. "The effects of $\mathrm{CO} 2$ and $\mathrm{H} 2$ on $\mathrm{CO}$ metabolism by pure and mixed microbial cultures." Biotechnology for biofuels 10.1 (2017): 220.

[54] Nam, Chul Woo, Kyung A. Jung, and Jong Moon Park. "Biological carbon monoxide conversion to acetate production by mixed culture." Bioresource technology 211 (2016): 478485 .

[55] Sharak Genthner B and Bryant M. Growth of Eubacterium limosum with carbon monoxide as the energy source. Appl Environ Micriobiol. (1982);43:

[56] Najafpour, Ghasem, and Habibollah Younesi. "Ethanol and acetate synthesis from waste gas using batch culture of Clostridium ljungdahlii." Enzyme and microbial technology38.1-2 (2006): 223-228.

[57] Kim, Byung Hong, et al. "Control of carbon and electron flow in Clostridium acetobutylicum fermentations: utilization of carbon monoxide to inhibit hydrogen production and to enhance butanol yields." Applied and environmental microbiology 48.4 (1984): 764-770.

[58] Phillips, John, Raymond Huhnke, and Hasan Atiyeh. "Syngas fermentation: a microbial conversion process of gaseous substrates to various products." Fermentation 3.2 (2017): 28

[59] Jung, Kyung-Won, Soonjae Lee, and Young Jae Lee. "Synthesis of novel magnesium ferrite $(\mathrm{MgFe} 2 \mathrm{O} 4) /$ biochar magnetic composites and its adsorption behavior for phosphate in aqueous solutions." Bioresource technology 245 (2017): 751-759.

[60] Huggins, Tyler M., et al. "Granular biochar compared with activated carbon for wastewater treatment and resource recovery." Water research 94 (2016): 225-232.

[61] Pan, Min, et al. "Kinetic, equilibrium and thermodynamic studies for phosphate adsorption on aluminum hydroxide modified palygorskite nano-composites." RSC Advances 7.8 (2017): 4492-4500.

[62] Jiang, Dan, Yoshimasa Amano, and Motoi Machida. "Removal and recovery of phosphate from water by calcium-silicate composites-novel adsorbents made from waste glass and shells." Environmental Science and Pollution Research 24.9 (2017): 8210-8218.

[63] Li, Ronghua, et al. "Recovery of phosphate from aqueous solution by magnesium oxide decorated magnetic biochar and its potential as phosphate-based fertilizer substitute." Bioresource technology 215 (2016): 209-214.

[64] Micháleková-Richveisová, Barbora, et al. "Iron-impregnated biochars as effective phosphate sorption materials." Environmental Science and Pollution Research 24.1 (2017): 463475. 
[65] Cai, Ru, et al. "Phosphate reclaim from simulated and real eutrophic water by magnetic biochar derived from water hyacinth." Journal of environmental management 187 (2017): 212219.

[66] Li, Ruining, et al. "Magnetic biochar-based manganese oxide composite for enhanced fluoroquinolone antibiotic removal from water." Environmental Science and Pollution Research 25.31 (2018): 31136-31148.

[67] Chen, Baoliang, Zaiming Chen, and Shaofang Lv. "A novel magnetic biochar efficiently sorbs organic pollutants and phosphate." Bioresource technology 102.2 (2011): 716-723.

[68] Reza, M. Toufiq, et al. "Production, characterization, and biogas application of magnetic hydrochar from cellulose." Bioresource technology 186 (2015): 34-43.

[69] Wang, Shengsen, et al. "Removal of arsenic by magnetic biochar prepared from pinewood and natural hematite." Bioresource technology 175 (2015): 391-395.

[70] Yang, Qi, et al. "Effectiveness and mechanisms of phosphate adsorption on iron-modified biochars derived from waste activated sludge." Bioresource technology 247 (2018): 537-544.

[71] Babu, Neelesh, and Vinay Mohan Pathak. "Biosorption of Heavy Metals: Biological Approach to Control the Industrial Waste." Handbook of Research on Microbial Tools for Environmental Waste Management. IGI Global, 2018. 270-281.

[72] Castro-Longoria, E., Alfredo R. Vilchis-Nestor, and M. Avalos-Borja. "Biosynthesis of silver, gold and bimetallic nanoparticles using the filamentous fungus Neurospora crassa." Colloids and Surfaces B: Biointerfaces 83.1 (2011): 42-48.

[73] Mukherjee, 4P, et al. "Green synthesis of highly stabilized nanocrystalline silver particles by a non-pathogenic and agriculturally important fungus T. asperellum." Nanotechnology 19.7 (2008): 075103.

[74] Borkovich, Katherine A., et al. "Lessons from the genome sequence of Neurospora crassa: tracing the path from genomic blueprint to multicellular organism." Microbiology and molecular biology reviews 68.1 (2004): 1-108.

[75] Vogel, Henry J. "Distribution of lysine pathways among fungi: evolutionary implications." The American Naturalist 98.903 (1964): 435-446.

[76] Cockrell, A. L.; Pirlo, R. K.; Babson, D. M.; Cusick, K. D.; Soto, C. M.; Petersen, E. R.; Davis, M. J.; Hong, C. I.; Lee, K.; Fitzgerald, L. A.; Biffinger, J. C. Suppressing the Neurospora crassa Circadian Clock while Maintaining Light Responsiveness in Continuous Stirred Tank Reactors. Sci. Rep. 2015, 5, 10691

[77] Ayawei, Nimibofa, Augustus Newton Ebelegi, and Donbebe Wankasi. "Modelling and interpretation of adsorption isotherms." Journal of Chemistry 2017 (2017). 
[78] Ng, J.C.Y., W. H. Cheung, and G. McKay. "Equilibrium studies of the sorption of Cu (II) ions onto chitosan." Journal of Colloid and Interface Science 255.1 (2002): 64-74.

[79] Marquardt, Donald W. "An algorithm for least-squares estimation of nonlinear parameters." Journal of the society for Industrial and Applied Mathematics 11.2 (1963): 431441.

[80] Haas, H. "Molecular genetics of fungal siderophore biosynthesis and uptake: the role of siderophores in iron uptake and storage." Applied microbiology and biotechnology 62.4 (2003): 316-330.

[81] Rashmi, K., et al. "Bioremediation of 60Co from simulated spent decontamination solutions." Science of the total environment 328.1-3 (2004): 1-14.

[82] Li, Qianwei, Laszlo Csetenyi, and Geoffrey Michael Gadd. "Biomineralization of metal carbonates by Neurospora crassa." Environmental science \& technology 48.24 (2014): 1440914416.

[83] Machala, Libor, Jirí Tuček, and Radek Zboril. "Polymorphous transformations of nanometric iron (III) oxide: a review." Chemistry of materials 23.14 (2011): 3255-3272.

[84] Zhang, Ming, et al. "Preparation and characterization of a novel magnetic biochar for arsenic removal." Bioresource technology 130 (2013): 457-462.

[85] Tronc, E., et al. "Surface-related properties of $\gamma$-Fe 2 O 3 nanoparticles." Journal of Magnetism and Magnetic Materials 221.1 (2000): 63-79.

[86] Andrade, A. L., et al. "Synthesis and characterization of magnetic nanoparticles coated with silica through a sol-gel approach." Cerâmica 55.336 (2009): 420-424. ).

[87] Jubb, Aaron M., and Heather C. Allen. "Vibrational spectroscopic characterization of hematite, maghemite, and magnetite thin films produced by vapor deposition." ACS Applied Materials \& Interfaces 2.10 (2010): 2804-2812.

[88] Namduri, H., and S. Nasrazadani. "Quantitative analysis of iron oxides using Fourier transform infrared spectrophotometry." Corrosion Science 50.9 (2008): 2493-2497.

[89] Coates, John. "Interpretation of infrared spectra, a practical approach." Encyclopedia of analytical chemistry (2000).

[90] Lenntech."Water Treatment Solutions." Lenntech Water Treatment \& Purification, www.lenntech.com/phosphorous-removal.htm. (2019)

[91] Daou, T. J., et al. "Phosphate adsorption properties of magnetite-based nanoparticles." Chemistry of Materials 19.18 (2007): 4494-4505. 
[92] Lǘ, Jianbo, et al. "Adsorptive removal of phosphate by a nanostructured Fe-Al-Mn trimetal oxide adsorbent." Powder Technology 233 (2013): 146-154.

[93] Dada, A. O., et al. "Langmuir, Freundlich, Temkin and Dubinin-Radushkevich isotherms studies of equilibrium sorption of $\mathrm{Zn} 2+$ unto phosphoric acid modified rice husk." IOSR Journal of Applied Chemistry 3.1 (2012): 38-45.

[94] Zhang, $\mathrm{Yu}$, et al. "Arsenate adsorption on an $\mathrm{Fe}-\mathrm{Ce}$ bimetal oxide adsorbent: Role of surface properties." Environmental Science \& Technology 39.18 (2005): 7246-7253

[95] Zhang, Gaosheng, et al. "Removal of phosphate from water by a Fe-Mn binary oxide adsorbent." Journal of colloid and interface science 335.2 (2009): 168-174.

[96] $\mathrm{Li}$, Ting, et al. "La $(\mathrm{OH}) 3$-modified magnetic pineapple biochar as novel adsorbents for efficient phosphate removal." Bioresource technology 263 (2018): 207-213.

[97] Kortlever, Ruud, et al. "Catalysts and reaction pathways for the electrochemical reduction of carbon dioxide." The journal of physical chemistry letters 6.20 (2015): 4073-4082.

[98] Schouten, K. J. P., et al. "A new mechanism for the selectivity to C 1 and C 2 species in the electrochemical reduction of carbon dioxide on copper electrodes." Chemical Science 2.10 (2011): 1902-1909.

[99] Luan, Chuhao, et al. "High-performance carbon dioxide electrocatalytic reduction by easily fabricated large-scale silver nanowire arrays." ACS applied materials \& interfaces 10.21 (2018): 17950-17956.

[100] Kondratenko, Evgenii V., et al. "Status and perspectives of CO 2 conversion into fuels and chemicals by catalytic, photocatalytic and electrocatalytic processes." Energy \& environmental science 6.11 (2013): 3112-3135.

[101] Jens, Christian M., et al. "Rh-Catalyzed Hydrogenation of CO2 to Formic Acid in DMSObased Reaction Media: Solved and Unsolved Challenges for Process Development." Advanced Synthesis \& Catalysis 361.2 (2019): 307-316.

[102] Phillips, Katherine R., et al. "Sulfide-Derived Copper for Electrochemical Conversion of CO2 to Formic Acid." The journal of physical chemistry letters 9.15 (2018): 4407-4412.

[103] Koper, Marc TM. "Thermodynamic theory of multi-electron transfer reactions: Implications for electrocatalysis." Journal of Electroanalytical Chemistry 660.2 (2011): 254-260.

[104] Hori, Y. I. "Electrochemical CO 2 reduction on metal electrodes." Modern aspects of electrochemistry. Springer, New York, NY, 2008. 89-189.

[105] Hori, Yoshio, et al. "Electrocatalytic process of CO selectivity in electrochemical reduction of CO2 at metal electrodes in aqueous media." Electrochimica Acta 39.11-12 (1994): 1833-1839. 
[106] Hori, Yoshio, Katsuhei Kikuchi, and Shin Suzuki. "Production of CO and CH4 in electrochemical reduction of $\mathrm{CO} 2$ at metal electrodes in aqueous hydrogencarbonate solution." Chemistry Letters 14.11 (1985): 1695-1698.

[107] Kuhl, K. P.; Cave, E. R.; Abram, D. N.; Jaramillo, T. F. New insights into the electrochemical reduction of carbon dioxide on metallic copper surfaces. Energy Environ. Sci. 2012, 5 (5), 7050 .

[108] Shen, Jing, et al. "Electrocatalytic reduction of carbon dioxide to carbon monoxide and methane at an immobilized cobalt protoporphyrin." Nature communications 6 (2015): 8177.

[109] Shen, Jing, et al. "DFT study on the mechanism of the electrochemical reduction of CO2 catalyzed by cobalt porphyrins." The Journal of Physical Chemistry C 120.29 (2016): 1571415721 .

[110] Göttle, Adrien J., and Marc TM Koper. "Proton-coupled electron transfer in the electrocatalysis of $\mathrm{CO} 2$ reduction: prediction of sequential vs. concerted pathways using DFT." Chemical science 8.1 (2017): 458-465.

[111] Tripkovic, Vladimir, et al. "Electrochemical $\mathrm{CO} 2$ and $\mathrm{CO}$ reduction on metalfunctionalized porphyrin-like graphene." The Journal of Physical Chemistry C 117.18 (2013): 9187-9195.

[112] Landers, Alan T., et al. "The Predominance of Hydrogen Evolution on Transition Metal Sulfides and Phosphides under CO2 Reduction Conditions: An Experimental and Theoretical Study." ACS Energy Letters 3.6 (2018): 1450-1457.

[113] Costentin, Cyrille, et al. "A local proton source enhances $\mathrm{CO} 2$ electroreduction to $\mathrm{CO}$ by a molecular Fe catalyst." Science338.6103 (2012): 90-94.

[114] Mohamed, Eman A., Zaki N. Zahran, and Yoshinori Naruta. "Efficient electrocatalytic CO 2 reduction with a molecular cofacial iron porphyrin dimer." Chemical Communications51.95 (2015): 16900-16903.

[115] Hammouche, Mohamed, et al. "Chemical catalysis of electrochemical reactions. Homogeneous catalysis of the electrochemical reduction of carbon dioxide by iron (" 0 ") porphyrins. Role of the addition of magnesium cations." Journal of the American Chemical Society 113.22 (1991): 8455-8466.

[116] Bhugun, Iqbal, Doris Lexa, and Jean-Michel Savéant. "Catalysis of the electrochemical reduction of carbon dioxide by iron (0) porphyrins. Synergistic effect of Lewis acid cations." The Journal of Physical Chemistry 100.51 (1996): 19981-19985.

[117] Bhugun, Iqbal, Doris Lexa, and Jean-Michel Saveant. "Ultraefficient selective homogeneous catalysis of the electrochemical reduction of carbon dioxide by an iron (0) porphyrin associated with a weak Broensted acid cocatalyst." Journal of the American Chemical Society 116.11 (1994): 5015-5016. 
[118] Bhugun, Iqbal, Doris Lexa, and Jean-Michel Savéant. "Catalysis of the electrochemical reduction of carbon dioxide by iron (0) porphyrins: Synergystic effect of weak Brönsted acids." Journal of the American Chemical Society 118.7 (1996): 1769-1776.

[119] Miyamoto, Kaito, and Ryoji Asahi. "Cation Impact on Cobalt-Porphyrin Catalyzed Electrochemical Reduction of CO2." arXiv preprint arXiv:1806.10285 (2018).

[120] Costentin, Cyrille, et al. "Ultraefficient homogeneous catalyst for the CO2-to-CO electrochemical conversion." Proceedings of the National Academy of Sciences 111.42 (2014): 14990-14994.

[121] $\mathrm{Hu}$, Xin-Ming, et al. "Enhanced catalytic activity of cobalt porphyrin in $\mathrm{CO} 2$ electroreduction upon immobilization on carbon materials." Angewandte Chemie 129.23 (2017): 6568-6572.

[122] Zheng, Tingting, Kun Jiang, and Haotian Wang. "Recent Advances in Electrochemical CO2-to-CO Conversion on Heterogeneous Catalysts." Advanced Materials 30.48 (2018): 1802066

[123] Savéant, Jean-Michel. "Molecular catalysis of electrochemical reactions. Mechanistic aspects." Chemical Reviews 108.7 (2008): 2348-2378.

[124] Zhu, Dong Dong, Jin Long Liu, and Shi Zhang Qiao. "Recent advances in inorganic heterogeneous electrocatalysts for reduction of carbon dioxide." Advanced materials 28.18 (2016): 3423-3452.

[125] Nielsen, Ida MB, and Kevin Leung. "Cobalt- porphyrin catalyzed electrochemical reduction of carbon dioxide in water. 1. a density functional study of intermediates." The Journal of Physical Chemistry A 114.37 (2010): 10166-10173.

[126] Yao, Cang Lang, et al. "Cobalt-porphine catalyzed CO 2 electro-reduction: a novel protonation mechanism." Physical Chemistry Chemical Physics 19.23 (2017): 15067-15072.

[127] Leung, K., Nielsen, I. M. B., Sai, N., Medforth, C.\& Shelnutt, J. A.Cobalt-porphyrin catalyzed electrochemical reduction of carbon dioxide in water. 2. mechanism from first principles. J. Phys. Chem. A 114, 10174-10184 (2010)

[128] Essington, Michael E. Soil and water chemistry: an integrative approach. CRC press, 2015.

[129] Shannon, Robert D. "Revised effective ionic radii and systematic studies of interatomic distances in halides and chalcogenides." Acta crystallographica section A: crystal physics, diffraction, theoretical and general crystallography32.5 (1976): 751-767.

[130] Shen, Jing, Donghui Lan, and Tingjiao Yang. "Influence of Supporting Electrolyte on the Electrocatalysis of CO2 Reduction by Cobalt Protoporphyrin." INTERNATIONAL JOURNAL OF ELECTROCHEMICAL SCIENCE 13.10 (2018): 9847-9857.

[131] Weng, Zhe, et al. "Electrochemical CO2 reduction to hydrocarbons on a heterogeneous 
molecular $\mathrm{Cu}$ catalyst in aqueous solution." Journal of the American Chemical Society 138.26 (2016): 8076-8079.

[132] Team, ESRL Web. "ESRL Global Monitoring Division - Global Greenhouse Gas Reference Network.” ESRL Co2 Trends RSS, 1 Oct. 2005, www.esrl.noaa.gov/gmd/ccgg/trends/.

[133] "CO2 Statistics." Food and Agriculture Organization of the United Nations, www.fao.org/statistics/en/.

[134] Spokas, Kasparas, et al. "Assessment of policy impacts on carbon capture and sequestration and bioenergy for US'coal and natural gas power plants." AGU Fall Meeting Abstracts. 2017.

[135] Zhang, Tian. "More efficient together." Science 350.6262 (2015): 738-739.

[136] Nybo, S. Eric, et al. "Metabolic engineering in chemolithoautotrophic hosts for the production of fuels and chemicals." Metabolic engineering 30 (2015): 105-120.

[137] Naik, S. N, et al. "Production of first and second generation biofuels: a comprehensive review." Renewable and sustainable energy reviews 14.2 (2010): 578-597.

[138] Conrado, Robert J., and Ramon Gonzalez. "Envisioning the bioconversion of methane to liquid fuels." Science 343.6171 (2014): 621-623.

[139] Liao, James C., et al. "Fuelling the future: microbial engineering for the production of sustainable biofuels." Nature Reviews Microbiology 14.5 (2016): 288.

[140] Gimkiewicz, Carla, et al. "Study of electrochemical reduction of $\mathrm{CO} 2$ for future use in secondary microbial electrochemical technologies." ChemSusChem 10.5 (2017): 958-967.

[141] Doyle, Lucinda E., and Enrico Marsili. "Weak electricigens: a new avenue for bioelectrochemical research." Bioresource technology 258 (2018): 354-364.

[142] Torella, Joseph P., et al. "Efficient solar-to-fuels production from a hybrid microbialwater-splitting catalyst system." Proceedings of the National Academy of Sciences 112.8 (2015): 2337-2342.

[143] Wiechmann, A., and V. Müller. "Synthesis of acetyl-CoA from carbon dioxide in acetogenic bacteria." Biogenesis of Fatty Acids, Lipids and Membranes (2017): 1-18.

[144] Jack, Joshua, et al. "Directing Clostridium ljungdahlii fermentation products via hydrogen to carbon monoxide ratio in syngas." Biomass and Bioenergy 124 (2019): 95-101. 
[145] Pan, Yuan, et al. "Design of single-atom Co-N5 catalytic site: a robust electrocatalyst for $\mathrm{CO} 2$ reduction with nearly $100 \% \mathrm{CO}$ selectivity and remarkable stability." Journal of the American Chemical Society 140.12 (2018): 4218-4221.

[146] Philips, Jo, et al. "Biofilm formation by Clostridium ljungdahlii is induced by sodium chloride stress: experimental evaluation and transcriptome analysis." PLoS One 12.1 (2017): e0170406.

[147] Huang, He, et al. "CRISPR/Cas9-based efficient genome editing in Clostridium ljungdahlii, an autotrophic gas-fermenting bacterium." ACS synthetic biology 5.12 (2016): 13551361.

[148] Jouny, Matthew, Wesley Luc, and Feng Jiao. "General techno-economic analysis of CO2 electrolysis systems." Industrial \& Engineering Chemistry Research 57.6 (2018): 2165-2177.

[149] Ainscough, C., Peterson, D. \& Miller, E. Hydrogen Production Cost From PEM Electrolysis (2014);

production_cost_pem_electrolysis.pdf

[150] Weng, Zhe, et al. "Active sites of copper-complex catalytic materials for electrochemical carbon dioxide reduction." Nature communications 9.1 (2018): 415.

[151] Bulushev, Dmitri A., and Julian RH Ross. "Heterogeneous catalysts for hydrogenation of $\mathrm{CO} 2$ and bicarbonates to formic acid and formates." Catalysis Reviews 60.4 (2018): 566-593.

[152] Wang, Lu, et al. "Greening ammonia toward the solar ammonia refinery." Joule 2.6 (2018): 1055-1074.

[153] Martín, Antonio José, Tatsuya Shinagawa, and Javier Pérez-Ramírez. "Electrocatalytic reduction of nitrogen: from Haber-Bosch to ammonia artificial leaf." Chem (2018).

[154] Salomone, Fabio, et al. "Techno-economic modelling of a Power-to-Gas system based on SOEC electrolysis and CO2 methanation in a RES-based electric grid." Chemical Engineering Journal (2018).

[155] Gruber, Manuel, et al. "Power-to-Gas through thermal integration of high-temperature steam electrolysis and carbon dioxide methanation-Experimental results." Fuel Processing Technology 181 (2018): 61-74.

[156] Xiang, Z. P.; Deng, H. Q.; Peljo, P.; Fu, Z. Y.; Wang, S. L.; Mandler, D.; Sun, G. Q.; Liang, Z. X. Electrochemical Dynamics of a Single Platinum Nanoparticle Collision Event for the Hydrogen Evolution Reaction. Angew. Chem. Int. Ed. 2018, 57, 3464-3468

[157] Wang, G.; Parrondo, J.; He, C.; Li, Y.; Ramani, V. Pt/C/Ni(OH)2 Bi-Functional Electrocatalyst for Enhanced Hydrogen Evolution Reaction Activity under Alkaline Conditions. Journal of Electrochemical Society, 2017, 164 (13), 1307-1315. 
[158] Campos-Roldan, C. A.; Gonzalez-Huerta, R. G.; Alonso-Vante, N. The oxophilic and electronic effects on anchored platinum nanoparticles on $\mathrm{sp} 2$ carbon sites: The hydrogen evolution and oxidation reactions in alkaline medium. Electrochimica Acta, 2018, 283, 18291834.

[159] Lu, S.; Hu, Y.; Wan, S.; MaCaffrey, R.; Jin, Y.; Gu, H.; Zhang, W. Synthesis of Ultrafine and Highly Dispersed Metal Nanoparticles Confined in a Thioether-Containing Covalent Organic Framework and Their Catalytic Applications. J. Am. Chem. Soc. 2017, 139, 1708217088 .

[160] Jiang, Y.; Gao, Q. Heterogeneous Hydrogenation Catalyst over Recyclable $\operatorname{Pd}(0)$ Nanoparticle Catalysts Stabilized by PAMAM-SBA-15 Organic-Inorganic Hybrid Composite. $J$. Am. Chem. Soc. 2006, 128, 716-717.

[161] Liu, J.; He, K.; Wu, W.; Song, T. B.; Kanatzidis, M. G. In Situ Synthesis of Highly Dispersed and Ultrafine Metal Nanoparticles from Chalcogels. J. Am. Chem. Soc. 2017, 139, 2900-2903.

[162] Jiang, H. L.; Akita, T.; Ishida, T.; Haruta, M.; Xu, Q. Synergistic Catalysis of Au@Ag Core-Shell Nanoparticles Stabilized on Metal-Organic Framework. J. Am. Chem. Soc. 2011, 133, 1304-1306.

[163] Zhang, Z.; Shao, C.; Zou, P.; Zhang, P.; Zhang, M.; Mu, J.; Guo, Z.; Li, X.; Wang, C.; Liu, Y. In Situ assembly of well-dispersed gold nanoparticles on electrospun silica nanotubes for catalytic reduction of 4-nitrophenol. Chem. Comun., 2011, 47, 3906-3908.

[164] Varade, D.; Haraguchi, K. Synthesis of Highly Active and Thermaly Stable Nanostructured Pt/Clay Materials by Clay-Meditated in Situ Reduction. Langmuir 2013, 29, 1977-1984.

[165] Xiao, W.; Zhang, Y.; Liu, B. Raspberrylike SiO2@Reduced Graphene Oxide@AgNP Composite Microspheres with High Aqueous Dispersity and Excellent Catalytic Activity. ACS Appl. Mater. Interfaces 2015, 7, 6041-6046.

[166] Wei, J.; Wang, H.; Deng, Y.; Sun, Z.; Shi, L.; Tu, B. Luqman,; Zhao. Solvent Evaporation Induced Aggregating Assembly Approach to Three-Dimensional Ordered Mesoporous Silica with Ultralarge Accessible Mesopores. J. Am. Chem. Soc. 2011, 133, 20369-20377.

[167] Liu, S. H.; Wu, M. T.; Lai, Y. H.; Chaing, C. C.; Yu, N.; Liu, S. B. Fabrication and electrocatalytic performance of highly stable and active platinum nanoparticles supported on nitrogen-doped ordered mesoporous carbons for oxygen reduction reaction. J. Mater. Chem., 2011, 21, 12489-12496 
[168] Pachfule, P.; Panda, M. K.; Kandambeth, S.; Shivaprasad, S. M.; Diaz, D. D.; Banerjee, R. Multifunctional and robust covalent organic framework-nanoparticle hybrids. J. Mater. Chem. A. 2014, 2, 7944-7952.

[169] Ding, S. Y.; Gao, J.; Wang, Q.; Zhang, Y.; Song, W. G.; Su, C. Y.; Wang, W. Construction of Covalent Organic Framework for Catalysis: Pd/COF-LZU1 in Suzuki-Miyaura Coupling Reaction. J. Am. Chem. Soc. 2011, 133, 19816-19822.

[170] Chen, L.; Zhang, L.; Chen, Z.; Liu, H.; Luque, R.; Li, Y. A covalent organic frameworkbased route to the in situ encapsulation of metal nanoparticles in N-rich hollow carbon spheres. Chem. Sci., 2016, 7, 6015-6020.

[171] Bhadra, M.; Sasmal, H. S.; Basu, A.; Midya, S. P.; Kandambeth, S.; Pachfule, P.; Balaraman, E.; Banerjee, R. Predesigned Metal-Anchored Building Block for In Situ Generation of Pd Nanoparticles in Porous Covalent Organic Framework: Application in Heterogeneous Tandem Catalysis. ACS Appl. Mater. Interfaces 2017, 9, 13785-13792.

[172] Lin, S.; Diercks, C. S.; Zhang, Y. B.; Kornienko, N.; Nichols, E. M.; Zhao, Y.; Paris, A. R.; Kim, D.; Yang, P.; Yaghi, O. M.; Chang, C. J. Covalent organic frameworks comprising cobalt porphyrins for catalytic CO2 reduction in water. Science, 2015, 349 (6253), 1208-1213.

[173] Johnson, E. M.; Haiges, R.; Marinescu, S. C. Covalent-Organic Frameowkrs Composed of Rhenium Bipyridine and Metal Porphyrins: Designing Heterobimetallic Frameworks with Two Distinct Metal Sites. ACS Appl. Mater. Interfaces 2018, 10, 37919-37927.

[174] Wei J.; Zhou, M.; Long, A.; Xue, Y.; Liao, H.; Wei, C.; Xu, Z. J. Heterostructured Electrocatalysts for Hydrogen Evolution Reaction Under Alkaline Conditions. Nano-Micro Lett., $2018,10,75$.

[175] Munasinghe, Pradeep Chaminda, and Samir Kumar Khanal. "Biomass-derived syngas fermentation into biofuels." Biofuels. Academic Press, 2011. 79-98.

[176] Orgill, James J., et al. "A comparison of mass transfer coefficients between trickle-bed, hollow fiber membrane and stirred tank reactors." Bioresource technology 133 (2013): 340-346. 


\section{Appendix A}

\section{Appendix equations:}

Eq. (A.1): $\mathrm{CO}+\mathrm{H}_{2} \mathrm{O} \rightarrow \mathrm{CO}_{2}+2 \mathrm{H}^{+}+2 \mathrm{e}-$

Eq. (A.2): $\mathrm{H}_{2} \rightarrow 2 \mathrm{H}^{+}+2 \mathrm{e}-$

Eq. (A.3): acetyl-CoA $+\mathrm{CO}_{2}+2 \mathrm{H}^{+}+2 \mathrm{e}-\rightarrow \mathrm{CoA}+$ Pyruvate

Eq. (A.4): $4 \mathrm{CO}+2 \mathrm{H}_{2} \mathrm{O} \rightarrow \mathrm{CH}_{3} \mathrm{COOH}+2 \mathrm{CO}_{2}$

$\left(\Delta \mathrm{G}_{\mathrm{r}}^{\mathrm{O}}(\mathrm{kJ} / \mathrm{mol})=-175.04\right)$

Eq. (A.5): $6 \mathrm{CO}+3 \mathrm{H}_{2} \mathrm{O} \rightarrow \mathrm{CH}_{3} \mathrm{CH}_{2} \mathrm{OH}+4 \mathrm{CO}_{2}$

$\left(\Delta \mathrm{G}_{\mathrm{r}}^{\mathrm{O}}(\mathrm{kJ} / \mathrm{mol})=-224.75\right)$

Eq. (A.6): $11 \mathrm{CO}+5 \mathrm{H}_{2} \mathrm{O} \rightarrow \mathrm{C}_{4} \mathrm{H}_{10} \mathrm{O}_{2}+7 \mathrm{CO}_{2}$

$\left(\Delta \mathrm{G}_{\mathrm{r}}^{\mathrm{O}}(\mathrm{kJ} / \mathrm{mol})=-306.17\right)$

Eq. (A.7): $4 \mathrm{H}_{2}+2 \mathrm{CO}_{2} \rightarrow \mathrm{CH}_{3} \mathrm{COOH}+2 \mathrm{H}_{2} \mathrm{O}$

$\left(\Delta \mathrm{G}_{\mathrm{r}}^{\mathrm{O}}(\mathrm{kJ} / \mathrm{mol})=-94.92\right)$

Eq. (A.8): $6 \mathrm{H}_{2}+2 \mathrm{CO}_{2} \rightarrow \mathrm{CH}_{3} \mathrm{CH}_{2} \mathrm{OH}+3 \mathrm{H}_{2} \mathrm{O}$

$\left(\Delta \mathrm{G}_{\mathrm{r}}^{\mathrm{O}}(\mathrm{kJ} / \mathrm{mol})=-104.57\right)$

Eq. (A.9): $11 \mathrm{H}_{2}+4 \mathrm{CO}_{2} \rightarrow \mathrm{C}_{4} \mathrm{H}_{10} \mathrm{O}_{2}+6 \mathrm{H}_{2} \mathrm{O}$

Eq. (A.10): $[\mathrm{CoP}]^{0}+\mathrm{e}^{-} \rightarrow[\mathrm{CoP}]$

Eq. (A.11): $[\mathrm{CoP}]^{-}+\mathrm{CO}_{2} \rightarrow\left[\mathrm{Co}(\mathrm{P})-\left(\mathrm{CO}_{2}\right)\right]^{]}$

Eq. (A.12): $[\mathrm{CoP}]^{0}+\mathrm{CO}_{2}+\mathrm{H}^{+}+\mathrm{e}^{-} \rightarrow[\mathrm{CoP}-\mathrm{COOH}]^{0}$

Eq. (A.13): $\left[\mathrm{Co}(\mathrm{P})-\left(\mathrm{CO}_{2}\right)\right]^{-}+\mathrm{H}^{+} \rightarrow[\mathrm{Co}(\mathrm{P})-(\mathrm{COOH})]^{0}$

Eq. (A.14): $\left[\mathrm{Co}(\mathrm{P})-\left(\mathrm{CO}_{2}\right)\right]^{-}+\mathrm{H}^{+}+\mathrm{e}^{-} \rightarrow[\mathrm{Co}(\mathrm{P})-(\mathrm{COOH})]^{-}$

Eq. (A.15): $[\mathrm{Co}(\mathrm{P})-(\mathrm{COOH})]^{0}+\mathrm{H}^{+}+\mathrm{e}^{-} \rightarrow[\mathrm{Co}(\mathrm{P})-(\mathrm{CO})]^{0}+\mathrm{H}_{2} \mathrm{O}$

Eq. (A.16): $[\mathrm{Co}(\mathrm{P})]+\mathrm{CO}_{2}+\mathrm{H}^{+}+\mathrm{e}^{-} \rightarrow[\mathrm{Co}(\mathrm{P})-(\mathrm{OCHO})]^{0}$ 
Eq. (A.17): $\left[\mathrm{Co}(\mathrm{P})-\mathrm{CO}_{2}\right]+\mathrm{H}^{+}+\mathrm{e}^{-} \rightarrow[\mathrm{Co}(\mathrm{P})-(\mathrm{OCHO})]^{-}$

Eq. (A.18): $[\mathrm{Co}(\mathrm{P})-(\mathrm{OCHO})]^{-}+\mathrm{H}^{+} \rightarrow[\mathrm{CoP}]+\mathrm{HCOOH}$

Eq. (A.19): $[\mathrm{Co}(\mathrm{P})-(\mathrm{OCHO})]^{-}+\mathrm{H}^{+}+\mathrm{e}^{-} \rightarrow[\mathrm{CoP}]+\mathrm{HCOOH}$

Eq. (A.20): $[\mathrm{Co}(\mathrm{P})-(\mathrm{OCHO})]^{-}+\mathrm{H}^{+}+\mathrm{e}^{-} \rightarrow\left[\mathrm{Co}(\mathrm{P})-\left(\mathrm{OCH}_{2} \mathrm{O}\right)\right]^{-}$

Eq. (A.21): $[\mathrm{CoP}]^{0}+\mathrm{H}^{+}+\mathrm{e}^{-} \rightarrow[\mathrm{Co}(\mathrm{P})-(\mathrm{H})]^{0}$

Eq. (A.22): $[\mathrm{CoP}]^{-}+\mathrm{H}^{+}+\mathrm{e}^{-} \rightarrow[\mathrm{Co}(\mathrm{P})-(\mathrm{H})]^{-}$

Eq. (A.23): $\left[\mathrm{CoP}-\mathrm{CO}_{2}\right]^{-}+\mathrm{Na}^{+}\left(\mathrm{H}_{2} \mathrm{O}\right)_{5}+\mathrm{e}^{-} \rightarrow[\mathrm{Co}-94 \mathrm{COOH}]^{-}+\mathrm{Na}^{+}\left(\mathrm{H}_{2} \mathrm{O}\right)_{4} \mathrm{OH}^{-}$

Eq. (A.24): $\left[\mathrm{CoP}-\mathrm{CO}_{2}\right]^{-}+\mathrm{H}_{2} \mathrm{O}+\mathrm{e}^{-} \rightarrow[\mathrm{CoP}-\mathrm{COOH}]^{-}+\mathrm{OH}^{-}$ 


\section{Appendix tables:}

Table A.1: Henry's law constants and electrochemical half-cell potentials of reduced gas species

\begin{tabular}{|l|l|l|}
\hline $\begin{array}{l}\text { Gaseous } \\
\text { Substrate }\end{array}$ & $\mathbf{K}_{\mathbf{H}}{ }^{\mathbf{1}},(\mathbf{m M} / \mathbf{a t m})$ & $\begin{array}{l}\mathbf{E}_{\mathbf{0}}{ }^{\mathbf{2}}, \\
(\mathbf{m V})\end{array}$ \\
\hline $\mathrm{CO}$ & 7.40 & -581 \\
\hline $\mathrm{H}_{2}$ & 0.78 & -420 \\
\hline
\end{tabular}

1) Henry's Law constant under standard conditions ( $\mathrm{T}=298.15 \mathrm{~K})$ [10]

2) Electrochemical half cell potentials under standard conditions ( $\mathrm{T}=298.15, \mathrm{P}=1 \mathrm{~atm}$, Conc. $=1 \mathrm{M}$ ) calculated for Eq. A.7 and A.8, respectively 


\section{Appendix figures:}

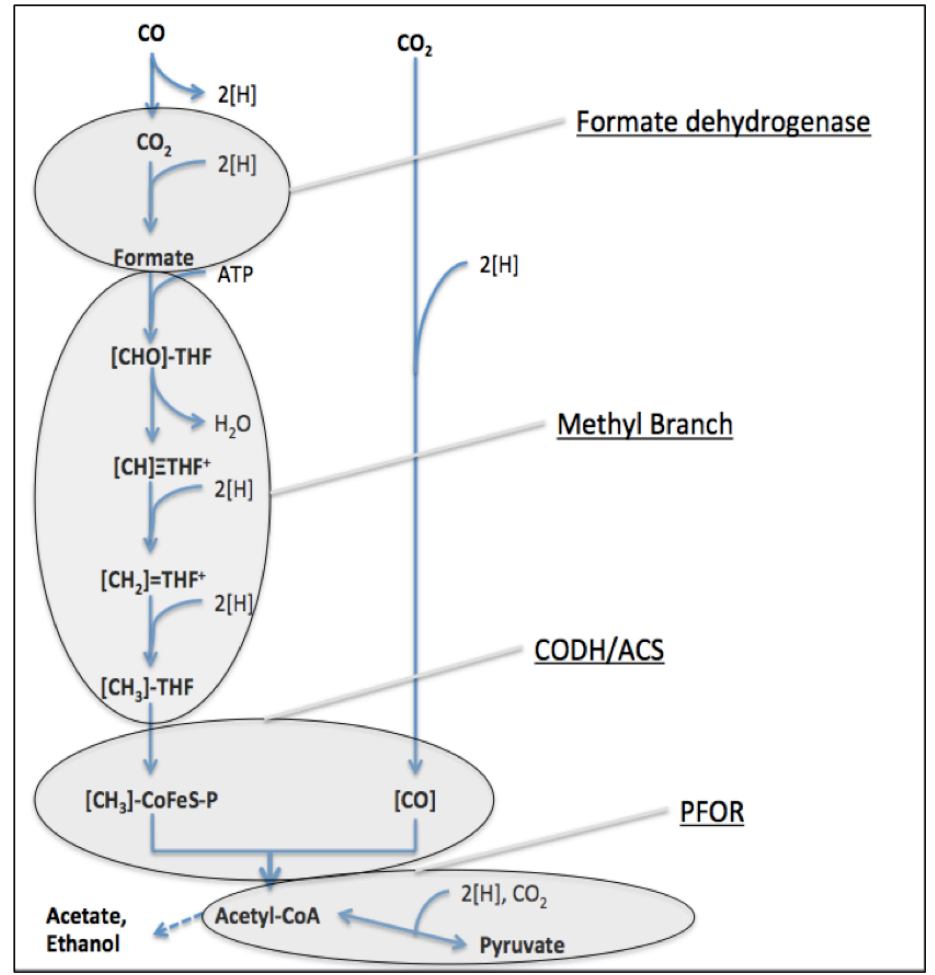

Figure A.1: Wood-ljungdahl pathway- Formation of acetyl-CoA from syngas components in western and eastern branches used for acetate, ethanol, and pyruvate production [21]

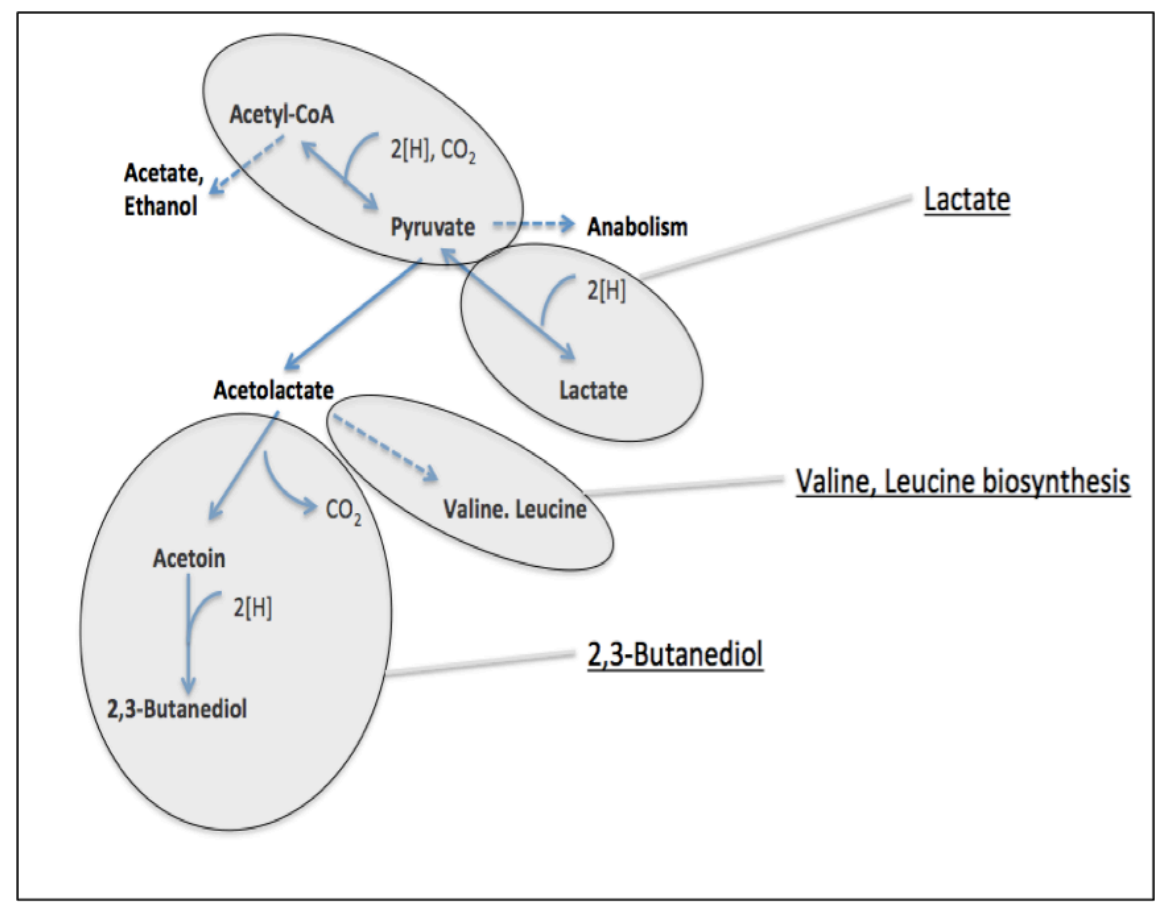


Figure A.2: Extended Wood-ljungdahl pathway- Conversion of Pyruvate to 2,3-Butanediol [22]

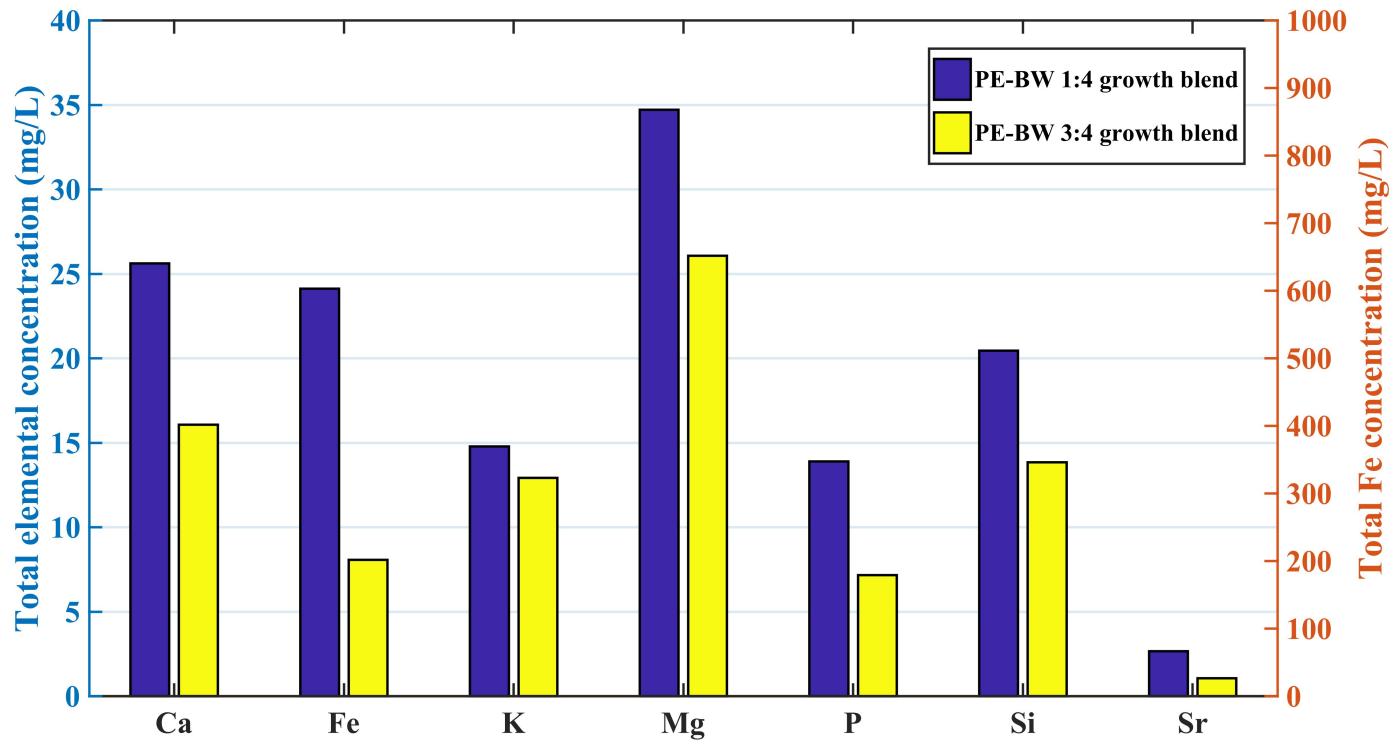

Figure A.3: Major constituents of growth blends used to generate fungal biomass as determined via ICP-MS analysis- right y-axis for total iron concentration only; Major constituents defined as initial concentration $>1 \mathrm{mg} / \mathrm{L}$.
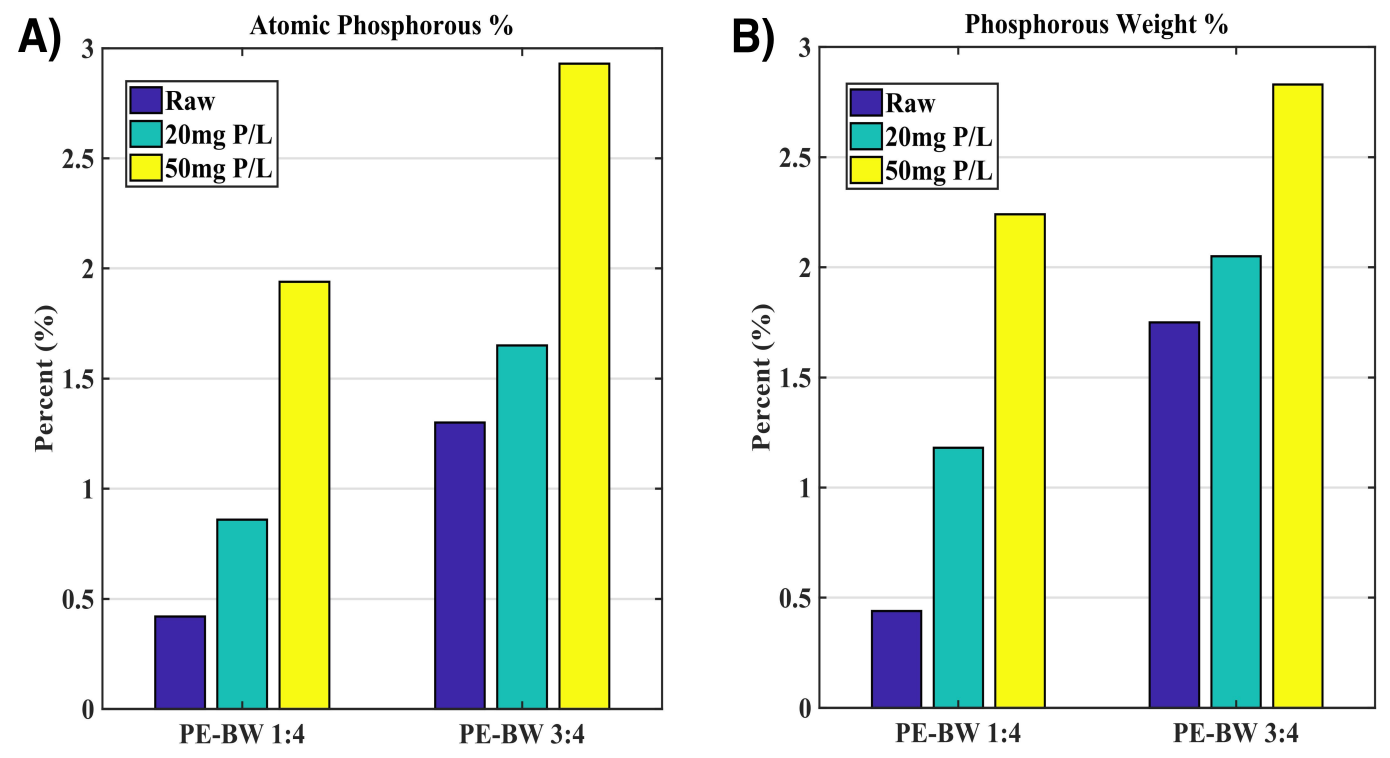
Figure A.4: Phosphorus loaded biochar EDS summary- Atomic phosphorous \% of PE-BW 1:4 and PE-BW 3:4 raw samples and after equilibrium in batch study with $20 \mathrm{mg}$ P/L and $50 \mathrm{mg}$ P/L (A) Phosphorous weight\% of PE-BW 1:4 and PE-BW 3:4 raw samples and after equilibrium in batch study with $20 \mathrm{mg} / \mathrm{L}$ and $50 \mathrm{mg}$ P/L (B)

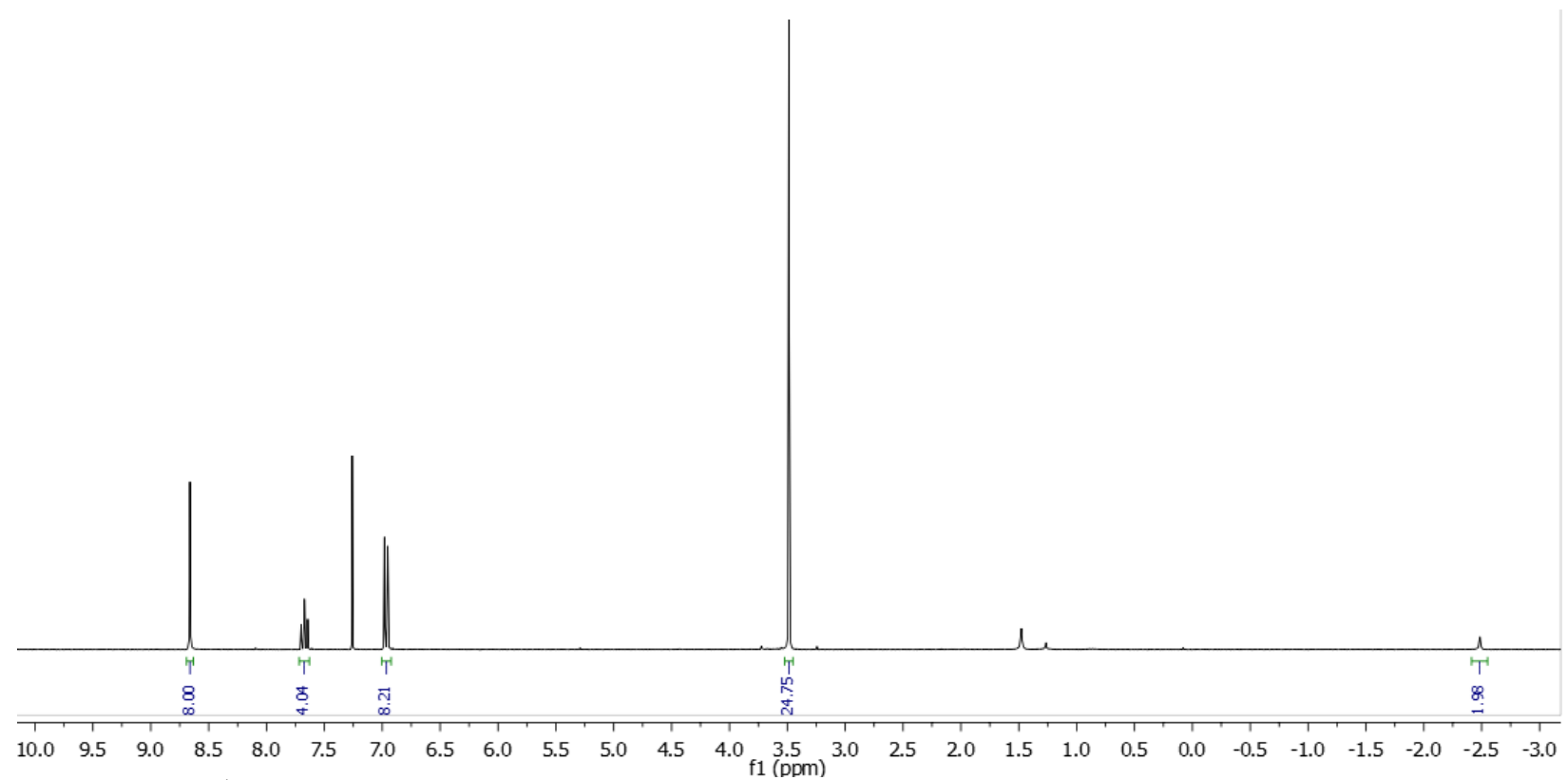

Figure A.5: ${ }^{1} \mathrm{H} \mathrm{NMR}\left(300 \mathrm{MHz}, \mathrm{CDCl}_{3}\right)$ of $\mathrm{H}_{2}\left(\mathrm{O}_{-}-\mathrm{OCH}_{3}\right) \mathrm{TPP}$

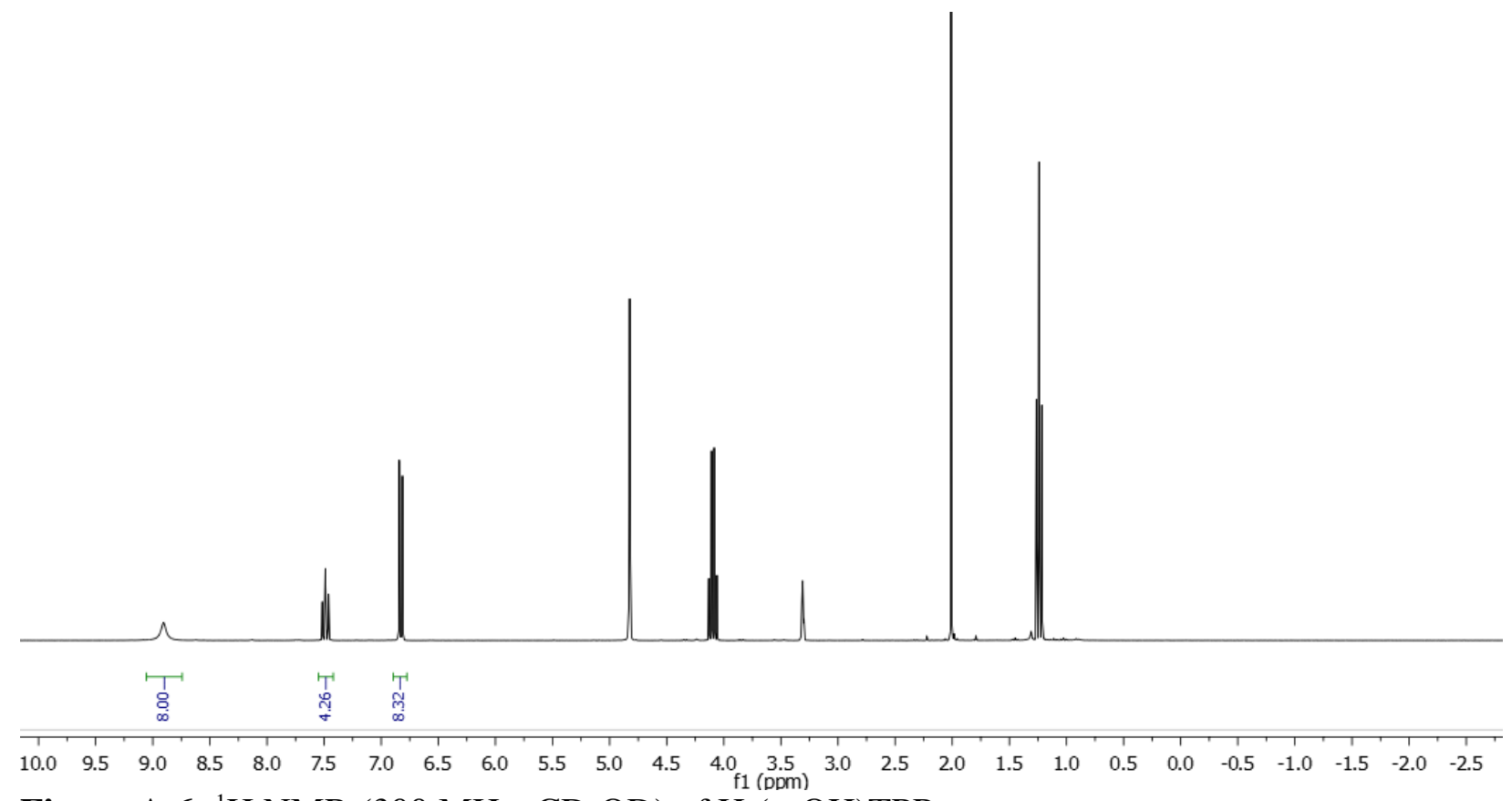

Figure A.6: ${ }^{1} \mathrm{H} \mathrm{NMR}\left(300 \mathrm{MHz}, \mathrm{CD}_{3} \mathrm{OD}\right)$ of $\mathrm{H}_{2}(\mathrm{O}-\mathrm{OH}) \mathrm{TPP}$ 


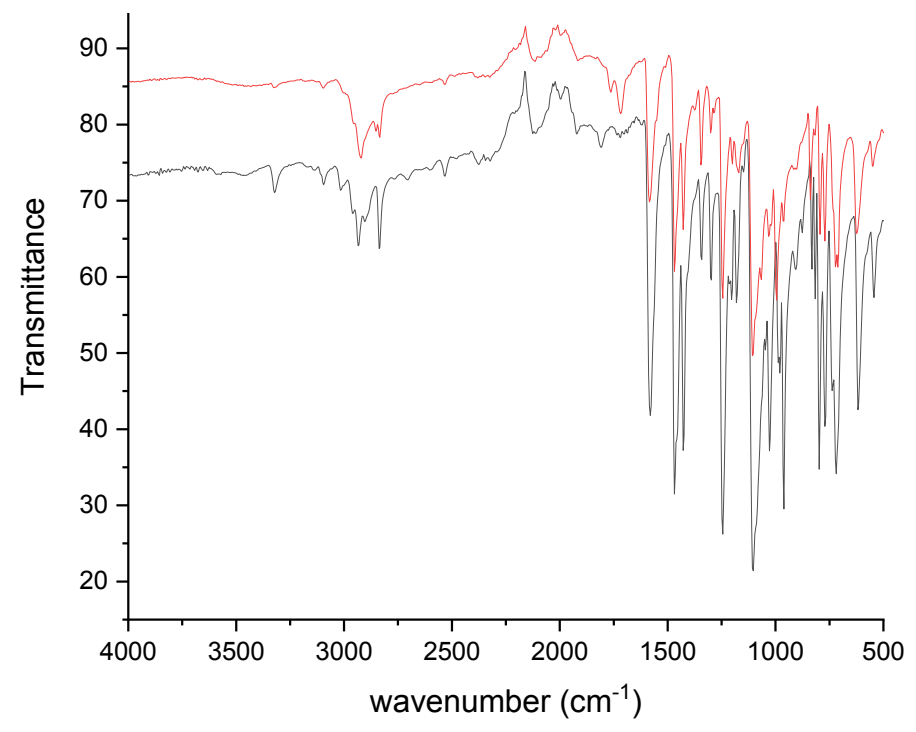

Figure A.7: FT-IR of $\mathrm{H}_{2}\left(\mathrm{o}-\mathrm{OCH}_{3}\right) \mathrm{TPP}$ (black) and $\mathrm{Co}\left(\mathrm{o}-\mathrm{OCH}_{3}\right) \mathrm{TPP}$ (red)

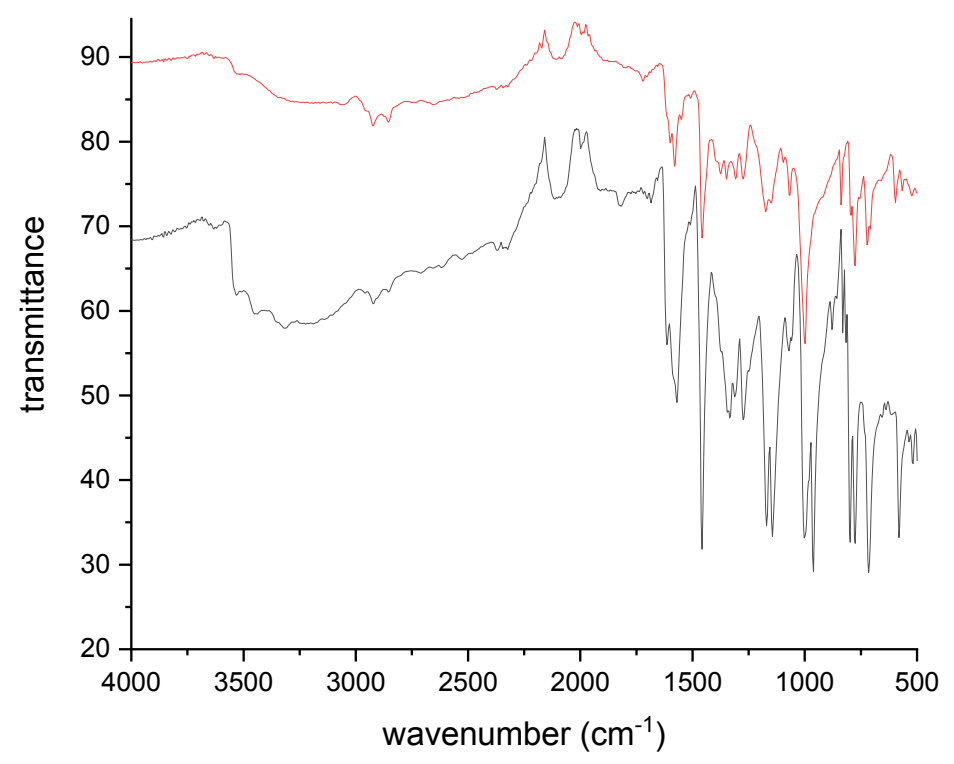

Figure A.8: FT-IR of $\mathrm{H}_{2}(\mathrm{o}-\mathrm{OH}) \mathrm{TPP}$ (black) and $\mathrm{Co}(\mathrm{o}-\mathrm{OH}) \mathrm{TPP}$ 


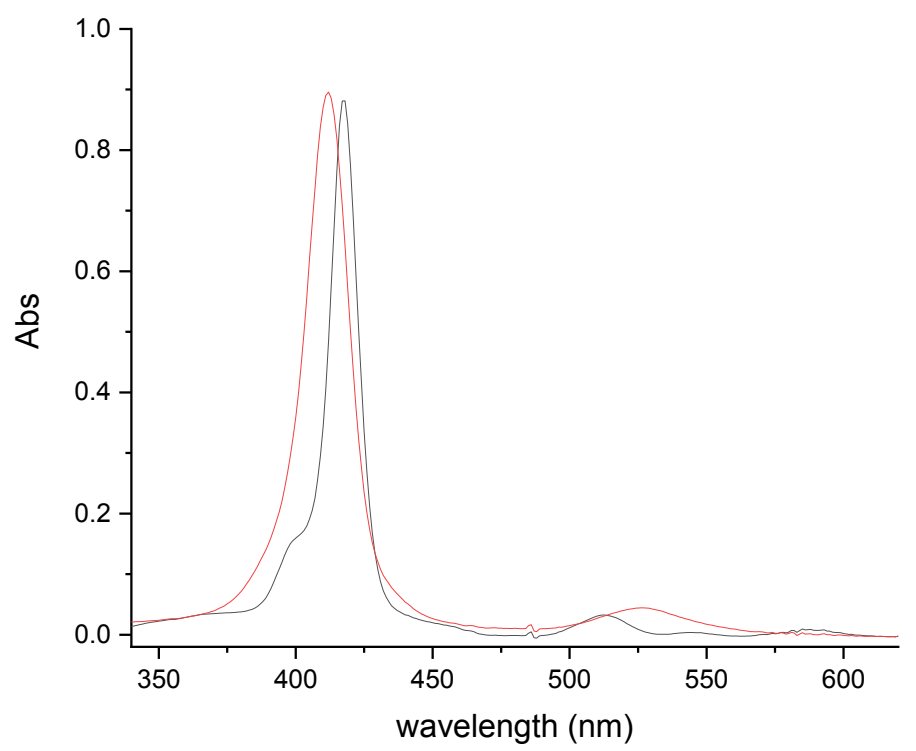

Figure A.9: UV-Vis in dichloromethane $\mathrm{H}_{2}\left(\mathrm{o}-\mathrm{OCH}_{3}\right) \mathrm{TPP}$ (black) and $\mathrm{Co}\left(\mathrm{o}-\mathrm{OCH}_{3}\right) \mathrm{TPP}$ (red)

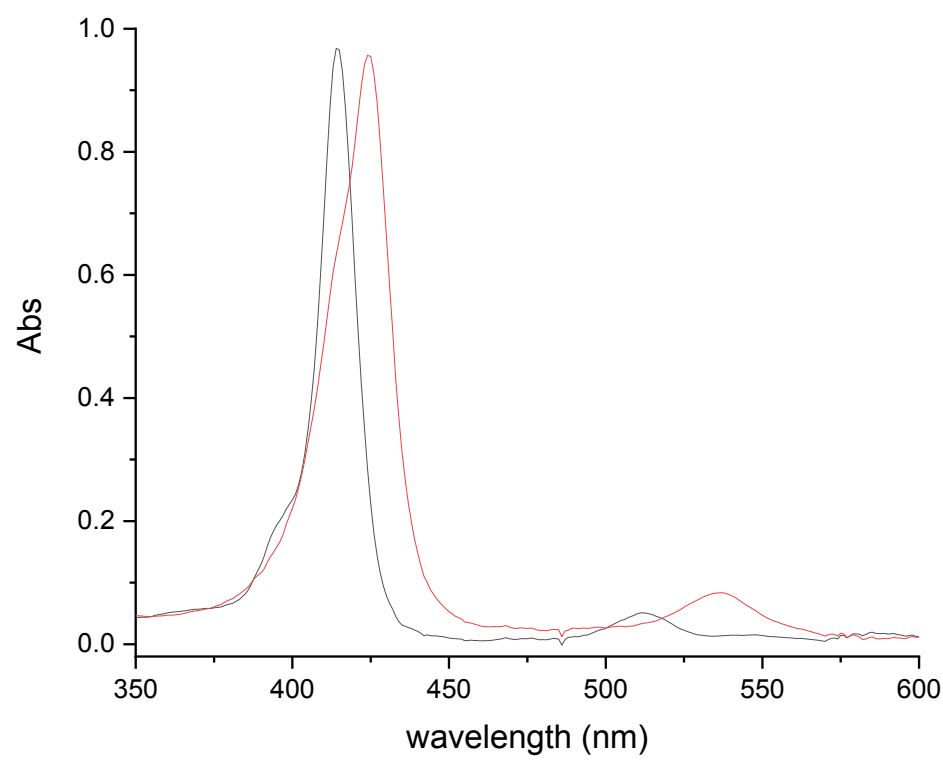

Figure A.10: UV-Vis of $\mathrm{H}_{2}(\mathrm{o}-\mathrm{OH}) \mathrm{TPP}$ (black) and $\mathrm{Co}(\mathrm{o}-\mathrm{OH}) \mathrm{TPP}$ in methanol 


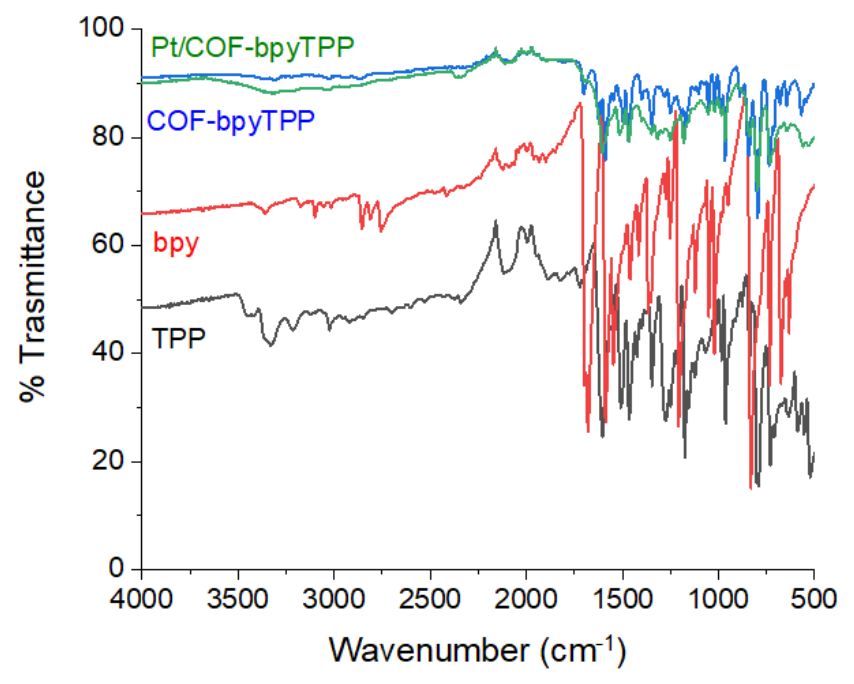

Figure A.11: FTIR of TPP, bpy, COF-bpyTPP, and Pt/COF-bpyTPP

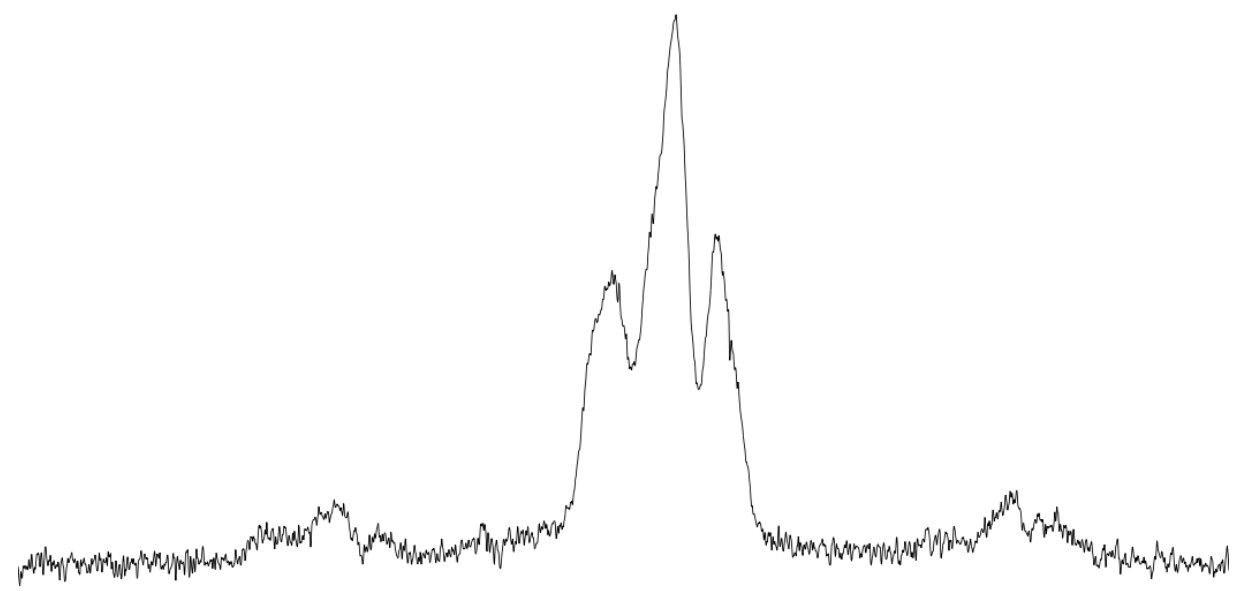

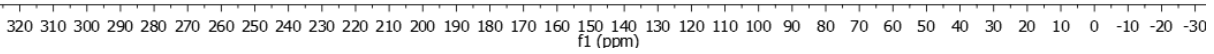
Figure A.12: Solid C NMR of COF-bpyTPP 


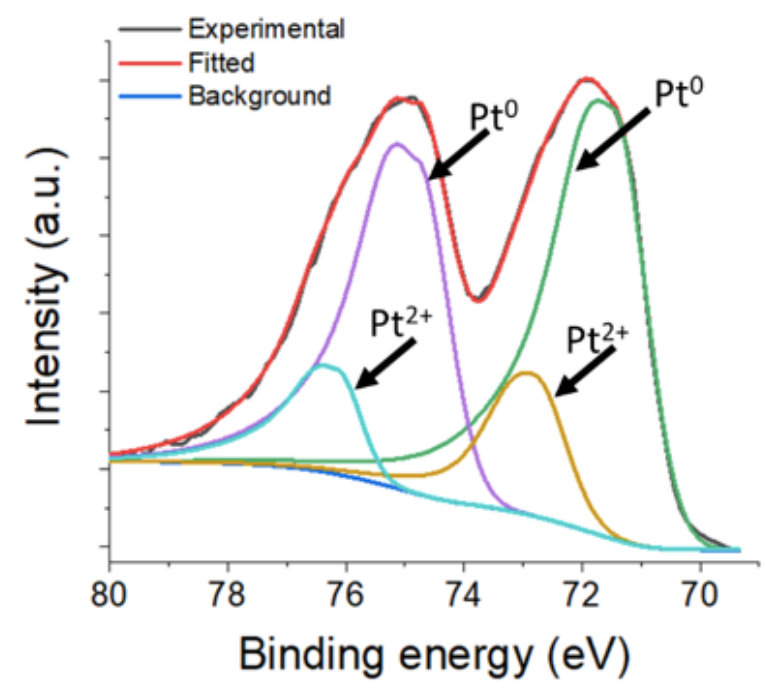

Figure A.13: XPS of Pt/COF-bpyTPP 


\section{Appendix supplemental methods:}

\section{$5,10,15,20$-tetrakis(2',6'-dimethoxyphenyl)-21H,23H-porphyrin.}

$\mathrm{BF}_{3} \mathrm{OEt}_{2}(0.13 \mathrm{~mL}, 1.08 \mathrm{mmol})$ was added to a mixture of 2,6-dimethoxybenzaldehyde $(1.2760 \mathrm{~g}$, $7.70 \mathrm{mmol})$ and pyrrole $(0.53 \mathrm{~mL}, 7.70 \mathrm{mmol})$ in chloroform $(750 \mathrm{~mL})$ under nitrogen. It was stirred at room temperature for 90 minutes in dark. After 2,3-dichloro-5,6-dicyano-1,4benzoquinone $(1.31 \mathrm{~g}, 5.78 \mathrm{mmol})$ was added, it was stirred at $65^{\circ} \mathrm{C}$ for 90 minutes. Once it cooled down to room temperature, triethylamine was injected to remove the excessive acid. The mixture was concentrated down. Silica gel column chromatography was done by dichloromethane. To obtain pure product, further purification was done with recrystallization in ethyl acetate. $21 \%$ yield $(0.3450 \mathrm{~g}) .{ }^{1} \mathrm{H}$ NMR $(300 \mathrm{MHz}, \mathrm{CDCl} 3) \delta=8.66(\mathrm{~s}, 8 \mathrm{H}), 7.67(\mathrm{t}, \mathrm{J}=8.4$ $\mathrm{Hz}, 4 \mathrm{H}), 6.96(\mathrm{~d}, \mathrm{~J}=8.4 \mathrm{~Hz}, 8 \mathrm{H}), 3.49$ (s, 24H), -2.49 (s, 2H). IR (cm $\left.{ }^{-1}\right): 3326,2934,2834$, $1584,1469,1428,1245,962 ; \lambda$ abs $\left(\mathrm{CH}_{2} \mathrm{Cl}_{2}\right): 418,514,546,589,643$.

\section{$5,10,15,20$-tetrakis(2',6'-dihydroxyphenyl)-21H,23H-porphyrin.}

A solution of $\mathrm{H}_{2}\left(\mathrm{o}-\mathrm{OCH}_{3}\right) \mathrm{TPP}(0.2 \mathrm{~g}, 0.234 \mathrm{mmol})$ in dry dichloromethane was placed in ice bath while boron bromide $(0.35 \mathrm{~mL}, 3.74 \mathrm{mmol})$ was added. The mixture was stirred at room temperature overnight. Ethyl acetate was added to suspension and it was washed with $\mathrm{NaHCO}_{3}$ (aq). Organic layer was separated and washed with water twice. Solvent was removed and pure violet compound was obtained. $66 \%$ yield $(0.1147 \mathrm{~g}) .{ }^{1} \mathrm{H}$ NMR $\left(300 \mathrm{MHz}, \mathrm{CD}_{3} \mathrm{OD}\right) \delta=8.92(\mathrm{~s}$, $8 \mathrm{H}), 7.50(\mathrm{t}, \mathrm{J}=8.2 \mathrm{~Hz}, 4 \mathrm{H}), 6.84(\mathrm{~d}, \mathrm{~J}=8.2 \mathrm{~Hz}, 8 \mathrm{H}) . \mathrm{IR}\left(\mathrm{cm}^{-1}\right): 3441,2920,2848,1580,1458$; $\lambda \operatorname{abs}\left(\mathrm{CH}_{3} \mathrm{OH}\right): 414,513,550,587,641$; ESI-MS $\left([\mathrm{M}+\mathrm{H}]^{+}\right)$calcd for $\mathrm{C} 44 \mathrm{H} 30 \mathrm{~N} 4 \mathrm{O} 8743.2142$, found 743.2135 .

\section{Cobalt (II) - 5,10,15,20-tetrakis(2',6'-dimethoxyphenyl)-21H,23H-porphyrin.}


Solid of $\mathrm{H}_{2}\left(\mathrm{o}-\mathrm{OCH}_{3}\right)$ TPP $(0.1453 \mathrm{~g}, 0.170 \mathrm{mmol})$ and anhydrous $\mathrm{CoCl}_{2}$ (II) $(0.441 \mathrm{~g}, 3.40 \mathrm{mmol})$ were dissolved in anhydrous THF $(30 \mathrm{~mL})$ under inert gas. 2.6-lutidine $(0.060 \mathrm{~mL}, 0.51 \mathrm{mmol})$ was added. The solution was refluxed for two hours. After removing THF solution, the mixture was dissolved in dichloromethane and washed with water three times. Organic solvent was concentrated down and product was collected. $85 \%$ yield $(0.132 \mathrm{~g}) . \mathrm{IR}\left(\mathrm{cm}^{-1}\right): 2920,2834,1584$, 1469, 1428, 1245; $\lambda$ abs $\left(\mathrm{CH}_{2} \mathrm{Cl}_{2}\right): 414,536$; ESI-MS $\left([\mathrm{M}+\mathrm{H}]^{+}\right)$calcd for $\mathrm{C}_{52} \mathrm{H}_{44} \mathrm{CoN}_{4} \mathrm{O}_{8} 911.88$, found 911.2484.

\section{Cobalt (II) - 5,10,15,20-tetrakis(2',6'-dihydroxyphenyl)-21H,23H-porphyrin.}

The same procedure to synthesize $\mathrm{Co}\left(\mathrm{o}-\mathrm{OCH}_{3}\right)$ TPP was followed except ethyl acetate to dissolve the mixture after removing THF solution. $\mathrm{H}_{2}(\mathrm{O}-\mathrm{OH}) \mathrm{TPP}(0.0573 \mathrm{~g}, 0.0772 \mathrm{mmol})$, anhydrous $\mathrm{CoCl}_{2}$ (II) $(0.200 \mathrm{~g}, 1.54 \mathrm{mmol}), 2,6$-lutidine $(0.028 \mathrm{~mL}, 0.2316 \mathrm{mmol})$, and anhydrous THF $(12 \mathrm{~mL})$ were used. $80 \%$ yield $(0.0494 \mathrm{~g}) . \mathrm{IR}\left(\mathrm{cm}^{-1}\right): 2920,2856,1580,1458 ; \lambda \mathrm{abs}\left(\mathrm{CH}_{3} \mathrm{OH}\right)$ : 426, 538; ESI-MS $\left([\mathrm{M}+\mathrm{H}]^{+}\right)$calcd for $\mathrm{C}_{44} \mathrm{H}_{28} \mathrm{CoN}_{4} \mathrm{O}_{8}$ 799.66, found 799 . 